\title{
Abstracts from HSG 2018
}

Published online: 12 October 2018

(C) The American Society for Experimental NeuroTherapeutics, Inc. 2018

1

Evaluating the Impact of Electroconvulsive Therapy (ECT) on Refractory Depression in Huntington Disease: a Case Series

\section{Contact:}

Jennifer Adrissi, MD

jennifer.smith1@northwestern.edu

301-502-0425

630 N. Franklin Street Unit 914

Chicago, IL 60654

United States

Jennifer Adrissi, Neil Nadkarni, Danny Bega

Northwestern University, Feinberg School of Medicine, Chicago, IL, USA (all authors)

Huntington disease (HD) is a progressive neurodegenerative disease characterized by involuntary movements and neuropsychiatric decline. With suicide rates five times higher in patients with HD compared with the general population, there is a need for further research into the management of affective symptoms in these patients. Electroconvulsive therapy (ECT) has long been used as a treatment for severe or medication-refractory mood disorders and catatonia. However, it is not the standard of care for the management of refractory symptoms in $\mathrm{HD}$, and there is a paucity of data and reported experiences with ECT in this population. Some case studies have demonstrated ECT's positive effect on depression and agitation in $\mathrm{HD}$, but the data are limited. In this single-site case series, we review three cases of ECT use for HD patients with refractory depression and suicidal ideation. One of these cases was associated with psychotic features. All three patients started treatments during an inpatient hospitalization with two continuing maintenance therapy as outpatients. All three had improvements in depression, agitation, and suicidal ideation leading to successful hospital discharges. One of the three patients also demonstrated subjective improvement in cognitive and motor symptoms after ECT initiation. This adds to the small body of case reports on the topic of ECT for HD. Larger-scale studies are warranted to further investigate the specific role and protocol for the use of ECT in the management of refractory depression and psychosis in this population.

2

\section{The Prionic Properties of Mutant Huntingtin Protein}

\section{Contact:}

Melanie Alpaugh

melanie.alpaugh@gmail.com

1-780-217-8799

301-962 Avenue Wilfrid-Pelletier

Quebec, QC G1W 4X8

Canada
Melanie Alpaugh ${ }^{1,2}$, Maria Masnata ${ }^{1,2}$, Alexander Maxan ${ }^{1,2}$, Giacomo Sciacca ${ }^{1,2}$, Hélena Denis ${ }^{1,2}$, Linda David ${ }^{1,2}$, Martine Saint-Pierre ${ }^{1,2}$, Luc Bousset ${ }^{3}$, Ronald Melki ${ }^{3}$, Francesca Cicchetti $^{1,2}$

${ }^{1}$ Centre de Recherche du CHU de Québec (CHUQ), Ax Neurosciences, 2705 Boulevard Laurier, Québec, QC, Canada, G1V 4G2; ${ }^{2}$ Département de Psychiatrie \& Neurosciences, Université Laval, Québec, QC, Canada, G1K 0A6; ${ }^{3}$ Paris-Saclay Institute of Neuroscience, CNRS, Gif-sur-Yvette, France

In recent years, evidence has accumulated suggesting that mutant huntingtin (mHtt) can spread into normal healthy tissue in a prion-like fashion. This theory, however, has remained controversial. To fully address this theory and to understand the possible effects of spreading on Huntington pathology, mice were administered exogenous human fibrillar mHtt (Q48) and Htt (Q25) Nterminal fragments using two distinct paradigms. Firstly, wildtype (WT) mice received a unilateral intracerebral injection at 2 months of age. Secondly, R6/2 and WT pups were administered fibrils by bilateral intraventricular injection. In both protocols, the injection of $\mathrm{mHtt}(\mathrm{Q} 48)$ fibrils resulted in behavioral changes. In the first protocol involving adult WT mice, a progressive cognitive impairment was observed in the open field beginning 10 months after treatment. In the second protocol involving R6/2 pups, a precipitation of phenotype was observed such that Q48-injected pups developed a motor (clasping and distance traveled in the open field) and anxiety-like (light-dark box) phenotype at 4 weeks of age. Postmortem analysis of adult WT mice indicated that most fibrils had been cleared from the brain by 12 months after surgery. Despite the lack of fibrils, a change in the staining pattern of endogenous huntingtin was detected. Interestingly, a similar change in the staining pattern was also present in postmortem analysis of the second protocol. Given the shorter time frame of the second protocol (12 weeks vs 12 months), it was possible to detect the presence of fibrils in the brain. The remaining fibrils were particularly concentrated in regions surrounding the lateral ventricles (the site of injection). Frequently, fibrils were found to colocalize with the endogenously present $\mathrm{mHtt}$ in $\mathrm{R} 6 / 2$ mice. The behavioral and postmortem findings together indicate that exogenously administered $\mathrm{mHtt}$ is capable of both inducing and exacerbating disease pathology in a prion-like manner.

Lay Summary: Recent findings have suggested that the abnormal protein in HD (mutant huntingtin) can move between cells. In order to better understand the effects of the movement of the abnormal protein, we treated normal and HD mice with synthetic mutant huntingtin. The normal mice developed memory problems, and the HD mice got sick faster. This suggests that targeting the huntingtin protein with antibody-based therapy could be beneficial in HD. 
3

Current State of Pharmacologic Approach to Huntington Chorea: Provider Survey from 32 HDSA Centers of Excellence

Contact:

Katherine Amodeo, MD

Movement Disorders Fellow

katherine_amodeo@urmc.rochester.edu

585-751-3400

601 Elmwood Avenue

Box MIND

Rochester, NY 14642

United States

Katherine Amodeo, Frederick Marshal

University of Rochester, Rochester, NY, USA (all authors)

Objective: Two vesicular monoamine transport type 2 inhibitors (VMAT2 inhibitors) - tetrabenazine and deutetrabenazine - are the only agents with Food and Drug Administration (FDA) approval for the management of chorea in Huntington disease (HD). The costs of VMAT-2 inhibitors are prohibitively high, and manufacturer-sponsored supports for tetrabenazine have vanished. Neuroleptics are often used clinically as an affordable alternative for managing chorea, but trials comparing their efficacy and side-effect profiles with VMAT-2 inhibitors are lacking. We report consensus opinion regarding current approaches and the need for head-to-head trials comparing neuroleptics with VMAT-2 inhibitors among Huntington Disease Society of America (HDSA) centers.

Methods: We sent an electronic-mailed survey to 32 center directors of HDSA Centers of Excellence across the USA. Directors were asked questions about their current approaches to chorea and proposed trial designs comparing neuroleptics with VMAT-2 inhibitors.

Results: The response rate was $100 \%$. Sixteen (50\%) favored the use of atypical neuroleptics, one (3\%) favored typical neuroleptics, and fifteen (47\%) favored the use of VMAT-2 inhibitors for first-line management of chorea. Of those favoring the use of atypical neuroleptics, affordability and treatment of comorbid mood disorders were the primary considerations. Among those favoring the use of VMAT-2 inhibitors as first-line management of chorea, the most commonly reported reason was the existence of data to support efficacy and side-effect profiles established in the clinical trials leading to FDA approvals. All were in favor of proceeding with a head-to-head trial to answer the question of whether atypical neuroleptics are comparable in the management of chorea, with most (34\%) proposing a randomized, double-blind clinical trial comparing an atypical versus a VMAT2-inhibitor as the most practical approach.

Conclusions: More than 50\% of surveyed providers use neuroleptics as first-line management for chorea over VMAT-2 inhibitors. Given a significant cost-discrepancy, studies comparing neuroleptics and VMAT-2 inhibitors are needed.

4

The Impact of Demographic Factors and Quality of Life on Cognitive Change in a Diverse Sample of Huntington Disease Patients

Contact:

Kendra M. Anderson

Clinical Neuropsychology Postdoctoral Fellow

kendra.m.anderson@uth.tmc.edu

870-872-7303

2255 Braeswood Park Dr. Apt. 311

Houston, TX 77030

United States
Kendra M. Anderson, Agustina Rossetti, Erin Furr-Stimming, Natalia Pessoa Rocha

McGovern Medical School at UTHealth, Houston, TX, USA (all authors)

Introduction: Huntington disease (HD) is expressed across multiple ethnic groups, yet little research has explored what factors possibly influence cognitive decline among ethnic minorities. Cognitive decline has been shown to negatively affect the quality of life in individuals with HD. However, it is unclear if this is a bidirectional relationship. Understanding lifestyle contributions (e.g., demographic factors) and patients' quality of life in the pathogenesis of HD is relevant to identifying interactions between overall well-being and disease course.

Methods: A longitudinal, retrospective cohort design was used for this study. Data were retrieved from the Enroll-HD database for 130 participants who were premanifest $(n=28)$ and manifest $(n=75) \mathrm{HD}$ gene carriers and identified as ethnic minorities (i.e., African American, Hispanic, Native American, or Asian) in North America. A weighted composite cognitive change score was calculated for each participant from the Symbol Digit Modalities Test (SDMT) and Stroop Word Reading Test. A hierarchical multiple regression was run to determine if the addition of demographic factors (marital status, education, area of residence, and employment status) and quality of life (physical component summary score (PCS) of the Short-Form Health Survey (SF-12)) improved the prediction of cognitive change beyond age and CAG repeats.

Results: The addition of demographic factors to the prediction of cognitive change led to a statistically significant increase in $R^{2}$ of $0.106, F(4$, $97)=2.404, p=0.05$, relative to age and CAG repeats alone. Specifically, marital status $(\mathrm{B}=-1.570, p=0.03)$ and employment $(B=-1.799, p=$ 0.02 ) were significant variables in the model. Adding a component of quality of life did not lead to a meaningful improvement in the prediction of cognitive change, as the final model was not statistically significant, $R^{2}=0.106, F(1,96)=0.007, p=0.935$.

Conclusions: Marital and occupational/vocational therapies may be preventative, nonpharmacological approaches to consider when working with diverse individuals diagnosed with HD.

5

Apathy Subtypes and the Functional Outcomes

Contact:

Kelly Atkins

kelly.atkins@monash.edu

61-399-051-918

987 Riversdale Road Surrey Hills

Melbourne 3127

Australia

Kelly Atkins, Trevor Chong, Sophie Andrews, Julie Stout

Monash Institute of Cognitive and Clinical Neuroscience, Monash University, Melbourne, Victoria, Australia (all authors)

Background: Apathy is a pervasive neuropsychiatric symptom of Huntington disease. Recently, three distinct subtypes of apathy have been proposed: cognitive (impairment in generating plans for action), emotional (blunting of feeling), and behavioral (reduced initiation of activity). To date, these subtypes have not been assessed in Huntington disease, and the unique impact of each on daily functioning is unclear.

Aims: The current study aims to assess cognitive, emotional, and behavioral apathy within a HD population and to examine associations between these subtypes and aspects of daily functioning.

Methods: To date, 21 premanifest and early HD participants and 26 genenegative controls have participated. They completed the Dimensional Apathy Scale (DAS), a self-report scale that assesses cognitive, emotional, 
and behavioral apathy, along with a self-report measure of depression, anxiety, and fatigue. They also completed a series of cognitive tasks and recorded their daily physical activity and sleep duration with a remote activity-tracking device (Fitbit one) over five consecutive days and nights. Results: The HD gene-positive group displayed significantly higher scores across all DAS subscales of apathy, compared with gene-negative controls. Higher cognitive apathy scores in the HD group were associated with poorer performance on a word-learning task $(r=-0.69, p=0.002)$. In contrast, emotional apathy scores in the HD group were associated with increased sleep duration $(r=0.46, p=0.035)$. For control participants, none of the three apathy subscales were correlated with sleep, activity, or cognition.

Conclusions: These preliminary results indicate that there are distinct subtypes of apathy in $\mathrm{HD}$, and that these subtypes affect daily functioning in different ways. Assessment and interventions that target each subtype of apathy, and the associated functional impact, are suggested to effectively manage the syndrome, rather than utilizing a "one size fits all" approach.

Understanding HD Psychosis: an Analysis from the Enroll-HD Database

Contact:

Danny Bega

dbega@nm.org

312-503-5706

710 N Lake Shore Drive

Chicago, IL 60657

United States

Ashwin Jaini, Chen Yeh, Danny Bega

Northwestern University Feinberg School of Medicine, Chicago, IL, USA (all authors)

Background: Huntington disease (HD) is an autosomal dominant neurodegenerative disorder characterized by progressive neuropsychiatric deterioration. Psychosis is thought to be rare in HD (prevalence of $3-$ $10 \%$ in various studies) patients but likely has a significant negative impact on quality of life and disease burden.

Objectives: This study aims to use a large database to determine the prevalence, onset, and severity of psychosis in $\mathrm{HD}$, and to determine what demographic and disease characteristics are associated with psychosis.

Methods: Data were obtained from Enroll-HD. Adults with manifest HD were included. Presence of psychosis was determined by investigator response to, "Has psychosis (hallucinations or delusions) ever been a part of the participant's medical history?" Descriptive statistics were calculated. Bivariate comparisons were conducted for demographic and disease characteristics between those with and without psychosis. Logistic regression was used to identify significant factors associated with psychosis. ORs with $95 \%$ CIs were calculated.

Results: Four thousand seven hundred twenty-seven manifest HD subjects were analyzed, and $11.72 \%$ had a history of psychosis. Mean age of onset of psychosis (47.6 years; S.D., 13.68) mirrored onset of HD. Family history of psychosis in a first-degree relative was documented in $99.23 \%$ of subjects with psychosis. Variables significantly $(p<0.05)$ associated with higher rates of psychosis in our multivariate model were non-White race (OR, 2.07), being single (OR, 2.45), or formerly married (OR, 1.87), lack of formal education beyond high school (OR, 1.67), history of violent behavior (OR, 2.03), history of apathy (OR, 1.84), and history of obsessive behaviors (OR, 2.11). The odds of psychosis increase by a factor of 1.10 for every 1 -unit decrease in the functional assessment score. Conclusions: Psychosis in HD is more common than many prior studies have reported. It is associated with worse functional outcomes. Our findings suggest several predictors that could be useful in improving our understanding of psychosis in this population.
Understanding Speech and Swallowing Difficulties in Individuals with Huntington Disease: Validation of the HDQLIFE Speech Difficulties and Swallowing Difficulties Item Banks

Contact:

Noelle E. Carlozzi

Associate Professor

carlozzi@med.umich.edu

734-763-8917

2800 Plymouth Road

Ann Arbor, MI 48109

United States

Presenter:

Nicholas R Boileau

Data Analyst

carlozzi@med.umich.edu

734-763-8917

2800 Plymouth Road

Ann Arbor, MI 48109

United States

N.R. Boileau ${ }^{1}$, A. Roberts ${ }^{2}$, D.L. Hanifan ${ }^{1}$, E. Mowers Provost ${ }^{2}$, P. Dayalu $^{2}$, J.A. Miner ${ }^{1}$, D. Claassen ${ }^{3}$, N.E. Carlozzi ${ }^{1}$

${ }^{1}$ University of Michigan, Ann Arbor, MI, USA; ${ }^{2}$ Northwestern University, Evanston, IL, USA; ${ }^{3}$ Vanderbilt University, Nashville, TN, USA

Aims: Speech and swallowing difficulties are among the most common symptoms in HD, occurring in $\sim 90 \%$ of affected individuals. These difficulties can have a significant impact on communication and social health-related quality of life (HRQOL). Recently, two new patientreported outcome (PRO) measures were developed to examine the impact that speech and swallowing difficulties have on HRQOL in persons with HD: HDQLIFE Speech Difficulties and HDQLIFE Swallowing Difficulties. The purpose of this analysis it to provide data to support the clinical utility of these two newly developed measures.

Methods: Thirty-one individuals with prodromal, early-, or late-stage HD and thirty-one matched controls completed these two new HDQLIFE measures, in addition to the Communication Participation Item Bank (CPIB). Participants also completed three traditional speech measures. Clinician ratings of dysarthria were also collected. A computational algorithm was also used to identify salient acoustic features of HD from audio recordings of persons with HD (average pauses, goodness of pronunciation, and words per second). Data were used to evaluate reliability (internal consistency), validity (known groups, convergent, and discriminant), and sensitivity/ specificity of the HDQLIFE Speech and Swallowing Difficulties item banks. Results: Cronbach's alphas were excellent for both of the HDQLIFE measures ( $\alpha$ 's $=0.95$ and 0.89 for speech and swallowing, respectively). Convergent validity was generally supported by moderate relationships among the PRO measures; discriminant validity supported correlations of lower magnitude with clinician and algorithm-generated features of speech. All PRO measures were also able to differentiate between controls, premanifest, early$\mathrm{HD}$, and late-HD participants supporting known groups validity. Classification accuracy was excellent. Each HDQLIFE measure was able to distinguish between clinician-rated dysarthria and no dysarthria (all AUC $\geq 0.87$ ).

Conclusions: Findings support the psychometric reliability and validity of the HDQLIFE Speech and Swallowing Difficulties measures in HD.

Lay Summary: As HD symptoms progress, speech and swallowing difficulties become more severe and can often impact health-related quality of life (HRQOL). Findings from this analysis indicate that these two new measures of speech and swallowing can be used to better understand the impact that speech and swallowing problems have on HRQOL in persons with HD. 
8

Safety and Tolerability of Research Lumbar Puncture in Huntington Disease: the HDClarity Cohort and Community Bioresource

Contact:

Filipe Brogueira Rodrigues, MD

f.rodrigues@ucl.ac.uk

00447484169226

UCL Huntington's Disease Centre

2nd floor, Russell Square House

10-12 Russell Square

London WC1B 5EH

United Kingdom

Filipe B. Rodrigues ${ }^{1}$, Anka Ehrhardt ${ }^{2}$, Gail Owen ${ }^{1}$, Stef Gosling ${ }^{1}$, Sherry $\mathrm{Lifer}^{2}$, Eileen $\mathrm{Neacy}^{2}$, Dipinder Kaur ${ }^{2}$, Olivia Handley ${ }^{2,3}$, Jenny Townhill $^{2,3}$, Blair Leavitt ${ }^{4}$, Mark Guttman ${ }^{5}$, Jee Bang ${ }^{6}$, Jamie Levey ${ }^{2,6}$, Elena $\mathrm{Pak}^{2}$, Cristina Sampaio ${ }^{2}$, Edward J. Wild ${ }^{1}$ (on behalf of the HDClarity investigators)

${ }^{1}$ UCL Huntington's Disease Centre, UCL Queen Square Institute of Neurology, London, UK; ${ }^{2}$ CHDI Management/CHDI Foundation, Princeton, NJ, USA; ${ }^{3}$ European Huntington's Disease Network, University Hospital of Ulm, Ulm, Germany; ${ }^{4}$ Centre for Molecular Medicine and Therapeutics, Department of Medical Genetics, University of British Columbia, Vancouver, BC, Canada; ${ }^{5}$ Division of Neurology, Department of Medicine, University of Toronto, Toronto, ON, Canada; ${ }^{6}$ Department of Neurology, Johns Hopkins University School of Medicine, Baltimore, MD, USA

With clinical trials ongoing and in planning to evaluate novel therapeutic approaches for Huntington disease (HD), biomarkers are needed to measure disease progression, target engagement, and efficacy. Cerebrospinal fluid (CSF) is an ideal medium to assess HD biomarkers and pathobiology in humans due to its proximity to the brain. There is a pressing need for a high-quality, international, open repository of CSF samples from well-characterized controls and HD gene-expansion carriers (HDGECs) spanning the disease spectrum.

HDClarity is the first of such project and aims to expedite HD research and therapeutic development. Its guiding principles are open collaboration, consistency in sample collection and processing, rigorous quality control, and the gathering of standardized, detailed phenotypic data via the EnrollHD platform. Each participant attends a screening and a sampling visit and may attend an optional repeat sampling visit $4-8$ weeks later.

Here, we report on the first 275 participants in HDClarity, recruited at 13 sites in 4 countries over 28 months. We present data on safety and tolerability of research lumbar puncture in controls and HDGECs from premanifest to advanced disease, as well as measures of CSF quality, collaborative research projects supported to date, and an overview of the sample application process. We outline future plans to introduce annual longitudinal sample collection and neuroimaging to HDClarity.

9

\section{Allele Selective Antisense Oligonucleotides for Reducing Mutant Huntingtin}

Contact:

Jeffrey Brown

Principal Scientist

jbrown@wavelifesci.com

617-949-4710

733 Concord Avenue

Cambridge, MA 02138

United States
Jeffrey M. Brown, Maria Frank-Kamenetsky, Kidist Aklilu, Jean-Cosme Dodart, Naoki Iwamoto, Zhong Zhong, Chandra Vargeese

Wave Life Sciences, 733 Concord Avenue, Cambridge MA 02138, USA (all authors)

Huntington disease (HD) is caused by cytosine-adenine-guanine (CAG) triplet repeat expansions in the HTT gene resulting in mutant HTT (mHTT) protein production. Nearly all HD patients are heterozygous for the expanded CAG repeat. Whereas the mHTT protein is causative for disease, preclinical studies suggest that loss of wild-type HTT (wtHTT) may contribute to neuronal damage. Therefore, the ability to selectively lower the production of $\mathrm{mHTT}$ protein while maintaining wtHTT protein levels holds great promise for HD treatment.

Antisense oligonucleotides (ASO) are short, modified nucleic acids designed to bind and degrade specific mRNA sequences. Standard chemical modifications to improve the stability and cellular uptake of ASOs include incorporation of a phosphorothioate (PS) backbone linkage and modification of the nucleotide sugar at the $2^{\prime}$ position. PS substitution converts each achiral phosphodiester (PO) linkage into a chiral PS center using one of two distinct stereochemical configurations, designated $S \mathrm{p}$ and $R \mathrm{p}$, at random. Through Wave's proprietary technology, we are able to control the three-dimensional structure of nucleic acid compounds to produce a more consistent molecular entity.

Using Wave's technology, we designed investigational ASOs to target a single nucleotide polymorphism (SNP) associated with the expanded CAG repeat on the $\mathrm{mHTT}$ allele. In vitro studies show that these ASOs preferentially target the $\mathrm{mHTT}$ transcript, while leaving the wtHTT mRNA largely intact in human neuronal systems. The ability of these ASOs to reduce mHTT mRNA is dependent on the specific positioning of the $R p$ stereochemistry. Using a defined stereochemistry, we were able to render an inactive ASO both active and selective against $\mathrm{mHTT}$. In addition to stereochemistry, specific patterns of $2^{\prime}$ modifications significantly improve the potency of the stereopure ASO in neuronal systems under free-uptake conditions, while maintaining selectivity for the $\mathrm{mHTT}$ mRNA. These results demonstrate that stereochemistry and 2 ' modifications are important for ASO design for allele targeting.

\section{0}

Creating a Career Research Coordinator: What Will Make This Generation of Coordinators Stay?

\section{Contact:}

Danielle Buchanan

Clinical and Translational Research Coordinator II

danielle.a.buchanan@vumc.org

615-875-3274

150021 st Ave S.

Nashville, TN 37232

United States

Danielle Buchanan ${ }^{1}$, Daniel Claassen ${ }^{1}$, Jody Goldstein ${ }^{2}$

${ }^{1}$ Vanderbilt University Medical Center, Nashville, TN, USA; ${ }^{2}$ University of Rochester, Rochester, NY, USA

Objective: This study aims to identify features of the Clinical Research Coordinator (CRC) position that could lead to greater career potential, less employee turnover, and higher overall vocational satisfaction by surveying current and former CRCs.

Background: Historically, a CRC position was revered as a long-term career with many staying in the position for 15-30 years. However, the culture of the position has shifted to being short term with a high turnover rate. This is 
problematic given the amount of time it takes to train a new individual for the position on the standard operating procedures, protocols, and other studyrelated materials. This information is advantageous to principle investigators (PIs) as it can enlighten them on what aspects of the position could change to make it a more desirable long-term career.

Design/Methods: Using survey method, information will be gathered identifying commonalities between former and current coordinators to analyze the aspects of the position that lead to fast turn-over rates and potential job dissatisfaction. Furthermore, data will be collected on ways to improve the position leading to higher vocational satisfaction and on making the position a long-term career option. The survey will utilize a combination of multiple-choice questions using Likert Scale levels of agreement. Data collection is currently underway, and a sample size of 50 is expected (ages 22-80).

Conclusion: Conclusions will summarize survey findings and indicate changes in the CRC position that would transform it into a long-term career. Given that Huntington disease trials are typically long term, it is better to have a coordinator that is with the family through the entire trial. Having a career coordinator will foster stability and support. Huntington disease affects an entire family, so all multidisciplinary clinical personnel involved need to be lasting including coordinators. CRCs have a unique role in the care and directly interact with patients and their family, making their role crucial.

\section{1}

\section{Parallel Evaluation of Mutant Huntingtin and Neurofilament Light as Biomarkers for Huntington Disease: the HD-CSF Study}

\section{Contact:}

Lauren M. Byrne

lauren.byrne.14@ucl.ac.uk

+447-709-524-351

Huntington's Disease Centre, UCL Institute of Neurology

2nd Floor Russell Square House, 10-12 Russell Square

London WC1B 5EH

United Kingdom

Lauren M. Byrne ${ }^{1}$, Filipe B. Rodrigues ${ }^{1}$, Eileanor B. Johnson ${ }^{1}$, Peter A. Wijeratne $^{2}$, Enrico De Vita ${ }^{3,4}$, Daniel C. Alexander ${ }^{2,5}$, Christian Czech ${ }^{6}$, Scott Schobel $^{6}$, Rachael I. Scahill ${ }^{1}$, Amanda Heslegrave ${ }^{7}$, Henrik Zetterberg ${ }^{7,8,9,10}$, Edward J. Wild ${ }^{1}$

${ }^{1}$ Huntington's Disease Centre, UCL Institute of Neurology, London, UK; ${ }^{2}$ Centre for Medical Image Computing, Department of Computer Science, UCL, London, UK; ${ }^{3}$ Lysholm Department of Neuroradiology, National Hospital for Neurology and Neurosurgery, London, UK; ${ }^{4}$ Department of Biomedical Engineering, School of Biomedical Engineering and Imaging Sciences, King's College London, UK; ${ }^{5}$ Clinical Imaging Research Centre, National University of Singapore, Singapore; ${ }^{6}$ Neuroscience, Ophthalmology, and Rare Diseases (NORD), Roche Pharma Research and Early Development, Roche Innovation Center Basel, F. Hoffman-La Roche Ltd., Basel, Switzerland; ${ }^{7}$ Department of Molecular Neuroscience, UCL Institute of Neurology, Queen Square, London, UK; ${ }^{8}$ UK Dementia Research Institute at UCL, London, UK; ${ }^{9}$ Department of Psychiatry and Neurochemistry, Institute of Neuroscience and Physiology, the Sahlgrenska Academy at the University of Gothenburg, Mölndal, Sweden; ${ }^{10}$ Clinical Neurochemistry Laboratory, Sahlgrenska University Hospital, Mölndal, Sweden

Background: Huntington disease (HD) is a progressive neurodegenerative disorder in which there is a pressing need for sensitive biomarkers. Aims: We assessed mutant huntingtin (mHTT) and neurofilament light (NfL) in parallel.
Methods: CSF mHTT, CSF NfL, and plasma NfL were measured using immunoassays in 80 participants (20 healthy controls, 20 premanifest HD, and 40 manifest HD) who underwent clinical assessments, and standardized CSF, and blood collections. Analysis included multiple linear regression models, Pearson's correlations, receiver operating characteristics curves, and sample size calculations. An event-based model was used to assess the temporal sequence of HD-related biomarker alterations.

Results: CSF mHTT, CSF NfL, and plasma NfL were significantly higher as disease progressed and were associated with all clinical measures. Both CSF and plasma NfL were associated with brain volume measures, but CSF mHTT was not. CSF mHTT, CSF NfL, and plasma NfL were closely correlated and highly stable within individuals. CSF mHTT had perfect accuracy for distinguishing between controls and HD mutation carriers, and both CSF and plasma NfL had excellent accuracy for distinguishing between premanifest and manifest HD. Sample size calculations suggest low participant numbers needed to incorporate these measures into clinical trials. The biofluid biomarkers emerged as the earliest detectable alterations in $\mathrm{HD}$, followed by brain volume and motor and cognitive measures.

Conclusion: In this cross-sectional study, we provide evidence to support mHTT and NfL as having favorable properties as biofluid biomarkers for HD. Our data suggest that these key biofluid biomarkers are some of the earliest detectable changes in HD.

\section{2}

\section{Enroll-HD Study Status}

\section{Contact:}

Selene Capodarca

selene@euro-hd.net

+39 3-934-418-988

Via De' Vanni 8

50142, Florence

Italy

Enroll-HD Platform Team

CHDI

Enroll-HD is a clinical research platform that includes at its core an observational, prospective study of HD. The objectives of Enroll-HD are to (1) enhance the design and expedite the conduct of clinical trials, (2) improve the understanding of the phenotypic spectrum and disease mechanisms, and (3) foster good clinical care.

More than 17,200 participants (15,466 currently active) have been recruited from 17 countries and 161 study sites. The data collected from these participants are monitored using a rigorous riskbased process. Recoded data and biosamples are made available to researchers. Enroll-HD also serves as a registry that can be used to facilitate recruitment, by identifying potentially eligible participants who can be invited by investigators to participate in clinical trials. Selection criteria (e.g., local CAG, UHDRS TMS) can be queried to generate listings of participants who are potentially eligible for a given clinical trial. To improve support for future clinical trials, the study will now start to prioritize the recruitment of premanifest and early-stage participants. Platform studies are clinical studies that utilize at least one or more Enroll-HD platform services. These services include site feasibility, study guidance documents and templates, potentially eligible participant listings, study set-up support, monitoring, and data management.

The Enroll-HD Clinical Training Portal is an online resource launched in January 2017 where HD research personnel can complete and maintain study-relevant training, presently UHDRS Motor Certification (all users) and good clinical practice (Enroll-HD Study users only). There are 
currently more than 1300 registered users, and it enables faster, more costeffective start-up of clinical trials and studies, standardizes the quality of training, and reduces workload at sites.

13

\section{Presymptomatic Testing for Huntington Disease}

\section{Contact:}

Erica Caporaletti

ecapor1@students.towson.edu

3-015-005-461

707 York Road Apt 3203

Towson, MD 21204

United States

\section{Erica Caporaletti, Froma Lustman}

Towson University, Towson, MD, USA (all authors)

Huntington disease (HD) is an autosomal dominant, neurodegenerative disease. When the genetic marker linked to HD was discovered, a presymptomatic test for this disease became available. Previous studies show that a vast majority of those "at-risk" do not test for the mutation that causes the disease; these studies show only $\sim 5-20 \%$ of individual test. The younger generation, however, appears to negate these statistics. An anonymous survey was posted on multiple Facebook support groups that targeted the population of those aged 18-30 who live in the USA and who are at-risk for HD.

Survey questions included whether the individual has undergone presymptomatic testing for HD or plans to do so in the future. After collecting 104 responses over the span of 3 weeks, the data were analyzed and showed that $\sim 43-59 \%$ of individuals aged 18-30 living in the USA test for $\mathrm{HD}$ at a $95 \%$ confidence interval. Of the 104 individuals who participated in this survey, 53 had tested for HD. Of the 51 individuals who had not tested, 28 participants answered that they would like to test in the future, whereas the remaining 23 participants said they were "unsure" if they wanted to test in the future. Not a single individual who participated in this survey indicated that they do not want to test for Huntington disease in the future.

The results of this survey affirm the importance of accurate reporting associated with HD. At-risk individuals, in fact, want information on the likelihood of their contracting this disease. If these individuals believe mistakenly that testing for $\mathrm{HD}$ is an option exercised infrequently, the value of the testing is perceived, at best, as being diminished, resulting in their being deterred from moving forward in getting tested.

\section{4}

24-Month Responsiveness of the HDQLIFE Physical, Mental, Cognitive, and Social Measures of Health-Related Quality of Life

\section{Contact:}

Noelle E. Carlozzi

carlozzi@med.umich.edu

734-763-8917

2800 Plymouth Road

Ann Arbor, MI 48109

United States

N.E. Carlozzi ${ }^{1}$, N.R. Boileau ${ }^{1}$, J.A. Miner ${ }^{1}$, J.S. Paulsen ${ }^{2}$, C.A. Ross ${ }^{3}$, J.S. Perlmutter ${ }^{4}$, M.K. McCormack ${ }^{5}$, E.A. Hahn ${ }^{6}$, J-S Lai ${ }^{6}$, M.A. Nance ${ }^{7}$, J. Stout ${ }^{8}$, S. Perlman ${ }^{9}$, S. Frank ${ }^{10}$, R. Ready ${ }^{11}$, S.K. Barton ${ }^{4}$, M. Wesson $^{12}$, D. Cella ${ }^{6}$, R.C. Gershon ${ }^{6}$, P. Dayalu ${ }^{1}$
${ }^{1}$ University of Michigan, Ann Arbor, MI USA ${ }^{2}$ The University of Iowa, Iowa City, IA, USA; ${ }^{3}$ Johns Hopkins University, Baltimore, MD, USA; ${ }^{4}$ Washington University School of Medicine, St. Louis, MO, USA; ${ }^{5}$ Rowan University, Piscataway, NJ, USA; ${ }^{6}$ Northwestern University, Evanston, IL, USA; ${ }^{7}$ Struthers Parkinson's Center, Golden Valley, MN, USA; ${ }^{8}$ Monash University, Clayton, Victoria, Australia; ${ }^{9}$ UCLA, Los Angeles, CA, USA F; ${ }^{10}$ Beth Israel Deaconess Medical Center, Boston, MA, USA; ${ }^{11}$ University of Massachusetts, Amherst, MA, USA;

${ }^{12}$ Indiana University, Indianapolis, IN, USA

Aims: Huntington disease (HD) is marked by progressive declines in physical, emotional, and cognitive abilities that impact health-related quality of life (HRQOL). A new measurement system, HDQLIFE, was previously developed and validated to examine HRQOL in HD, yet responsiveness data are still needed to support its clinical utility in longitudinal trials.

Methods: Two hundred fifty-six participants with HD $(n=86$ premanifest; $n=90$ early-HD; $n=80$ late HD) completed baseline and 24-month follow-up assessments. Participants completed several mental, physical, and social health measures from the HDQLIFE measurement system (which included three HD-specific measures (chorea, speech difficulties, and swallowing difficulties) and measures from the PROMIS and Neuro-QoL (anxiety, depression, anger, positive affect and well-being, emotional and behavioral dyscontrol, upper extremities function, lower extremities function, cognitive function-executive function, cognitive function-general concerns, ability to participate in social roles and activities, and satisfaction with social roles and activities)), as well as an in-person evaluation that included the UHDRS Total Functional Capacity (TFC). At 24 months, participants also completed several anchor items to assess self-reported changes in health. Twenty-four-month change in TFC scores was used to examine clinician-rated change. Standardized response means were used to evaluate whether the PROs were responsive to self-reported and clinician-rated change over time.

Results: Generally, we found small-to-moderate standardized response mean effect sizes from baseline to 24-month follow-up visits for those with worse self-reported functioning, as well as for those with clinicianrated functional decline; findings were the most robust for the physical and mental HRQOL measures.

Conclusions: Findings indicate that the HDQLIFE measurement system is sensitive to change over time in persons with Huntington disease.

15

\section{The Impact of Huntington Disease on Family Life}

Contact:

Phyllis Chua

phyllis.chua@monash.edu

61395941479

Department of Psychiatry, Faculty of Medicine, Nursing and Health Sciences, Monash University

Level 3, P Block, Monash Medical Centre, 246 Clayton Road, Clayton Clayton, Victoria 3168

Australia

Phyllis Chua ${ }^{1,2}$, Katya Kotschet ${ }^{1}$, Susan Mathers ${ }^{1}$, James Howe ${ }^{1}$, Yennie Lee $^{1}$, Sarah Lee ${ }^{1}$, Caron Chapman ${ }^{1}$, Katrina Reardon ${ }^{1}$, David Kissane ${ }^{2}$

${ }^{1}$ Statewide Progressive Neurological Services, Calvary Healthcare Bethlehem, South Caulfield, Victoria, Australia; ${ }^{2}$ Department of Psychiatry, Monash University, Clayton, Victoria, Australia

Michael J. Fox once said, "Family is not an important thing. It's everything." Families are often also carers. Carer burden is well recognized in many neurodegenerative diseases, including Huntington disease (HD). 
Psychosocial studies have focused on the individual with HD and their carers, but little research has been done on the impact on the family unit. The family systems theory can provide a useful framework to view the family unit. The family is considered a group of members who interact with each other according to rules that have evolved over time within a wider social context and over the life cycle (Sholevar and Perkel, 1990). A study by Vamos et al. (2007) using the Family Relationship Index (FRI) of 40 families with HD-reported scores in the dysfunctional range. A FRI cut-off score of $<9$ out of 12 is seen as indicative of "at-risk families" who could benefit from family-focused interventions.

This study will assess the quality of communication and relationships in families of patients with HD using the FRI and how this relates to the individual's disease stage and quality of life, as well as the carer's experience. Knowledge of these variables can assist in the identification of families who could benefit from early family-focused interventions to prevent family stress and potential breakdown.

Preliminary analysis of the FRI data from 35 patients with HD and their families have indicated a greater perception of family relationship difficulties by the family member (mean FRI, $8.59 \pm$ 2.95) than the person with HD (mean FRI, 9.44 \pm 2.36 ). These results suggest a need to consider the family unit and not just the patient and carer separately. We have now recruited a total of 70 patients with HD. We will report on the larger sample and the potential variables that could help identify these 'at-risk' families.

\section{6}

\section{Salivary Biomarkers for Huntington Disease}

\section{Contact:}

Jody Corey-Bloom

Professor of Neuroscience

jcoreybloom@ucsd.edu

858-249-0574

Department of Neurosciences

9500 Gilman Drive MC0948

La Jolla, CA 92093-0948

United States

Jody Corey-Bloom ${ }^{1}$, Ameera Haque ${ }^{1}$, Aeri Kim ${ }^{1}$, Sungmee Park ${ }^{1}$, Chase Snell $^{1}$, Douglas A Granger ${ }^{2}$, Steven W Granger ${ }^{3}$, Elizabeth A. Thomas ${ }^{4}$

${ }^{1}$ Department of Neurosciences, University of California San Diego, La Jolla, CA, USA ${ }^{2}$ Institute for Interdisciplinary Salivary Bioscience Research, UCI, Irvine, CA, USA ${ }^{3}$ Salimetrics, Carlsbad, CA, USA ${ }^{4}$ Department of Molecular and Cellular Neuroscience, The Scripps Research Institute, La Jolla, CA, USA

Objective: The objective of the current study was to assess the potential for saliva to serve as a biospecimen for accessible biomarkers for Huntington disease (HD).

Background: Peripheral biomarkers are greatly needed in HD to anticipate onset of disease symptoms, monitor progression, and track potential therapeutic effects. Pathogenesis in HD is associated with expression of the mutant $\mathrm{Htt}(\mathrm{mHtt})$ protein in the CNS; several therapeutic approaches directed at its production, processing, and/or turnover are under development. Recently, inflammatory molecules have been studied for various neurodegenerative diseases due to their role in neurodegeneration and immune system activation. Design/Methods: We measured total $\mathrm{Htt}(\mathrm{tH} t \mathrm{tt}$ ) protein in saliva from 98 manifest HD, gene-positive premanifest (PM) HD, and age- and sexmatched control (NC) subjects. Additional salivary analytes, including inflammatory markers, such as cortisol, C-reactive protein (CRP), interleukin-6 (IL-6), and interleukin-1B (IL-1B), were also measured using standardized ELISAs.
Results: Levels of tHtt were significantly higher in saliva from HD patients compared with NC $(p=0.02)$. Salivary tHtt showed no gender effects but showed a significant positive correlation with age but not age-of-onset or CAG-repeat length. Importantly, salivary tHtt was significantly correlated with several clinical measures - positively correlated with UHDRS TMS $(r=0.374, p=0.038)$, negatively correlated with UHDRS TFC score $(r=0.345 ; p=0.057)$, and negatively correlated with SDM score $(r=-0.424 ; p=0.017)$. CRP, a marker of systemic inflammation, was significantly elevated in saliva in PM subjects compared with $\mathrm{NC}(p=0.032)$ and may be an early marker for disease onset. Levels of salivary cortisol $(p=0.047)$ and IL-1B $(p=0.021)$ were significantly higher in HD compared with PM subjects. Levels of salivary IL-6 were significantly higher in HD compared with both PM $(p=0.048)$ and $\mathrm{NC}$ $(p=0.024)$ subjects.

Conclusions: Measurements of salivary $\mathrm{Htt}$ and other salivary proteins offer significant promise as relevant, noninvasive biomarkers of symptom onset and disease progression in HD.

\section{7}

Development of a Psychological Model of Anxiety Among Those AtRisk and Those Who Have Tested Positive for Huntington Disease

Contact:

Maria Dale, MD

Maria.Dale@leicspart.nhs.uk

01162951615

HD Community Team

Mill Lodge, The Rise, Narborough

Leicestershire LE19 4SL

United Kingdom

Maria Dale ${ }^{1}$, John Maltby ${ }^{2}$, Dawn Freire-Patino ${ }^{1}$, Reza Kiani ${ }^{1}$

${ }^{1}$ Leicestershire Partnership NHS Trust Mill Lodge, The Rise, Narborough, Leicestershire, UK; ${ }^{2}$ Centre for Medicine University of Leicester, Leicester, LE1 7EA, UK

Anxiety is common among both those who have tested positive for Huntington disease (HD) and those untested but at-risk for HD. Psychological interventions to help people with HD cope with anxiety, and the mechanisms that underlie anxiety in HD, are under-researched. Among the limited research, there are modifiable factors such as illness perceptions that are related to anxiety in $\mathrm{HD}$, which are amenable to psychological treatments. In order to develop an HD-specific psychological model of anxiety, a new scale was devised: the HD Concerns Questionnaire (HDCQ). A survey was published online via six international English-speaking HD organizations to examine HD-related worries, perceptions, and behaviors, as measured by the HDCQ. A total of 115 participants responded, comprising $64 \mathrm{HD}$ carriers and 51 adults at-risk of HD. The HDCQ was subjected to an exploratory factor analysis (EFA) to explore its structural validity. A maximum likelihood ratio EFA, with promax rotation, was undertaken, revealing a three-factor solution for the HDCQ: HD worries (four items), HD avoidance (three items), and HD anxiety (eight items). A series of spearman correlations revealed that greater HD worries, HD anxiety, and HD avoidance were all significantly related $(p=\leq 0.001)$ to higher levels of anxiety, as measured by the Hospital Anxiety and Depression Scale (HADS) anxiety subscale. Seventy-eight percent of respondents reported clinical levels of anxiety, as measured by scores $\geq 8$ on the HADS-A. No differences in anxiety across all HDCQ scales or the HADS-A were found across carriers or those at risk, indicating such issues to be also relevant to people who have decided not to be tested. These findings suggest a psychological model is a useful framework to address anxiety in those at-risk as well as those who tested positive for $\mathrm{HD}$. 
18

Presentation of Behavioral Symptoms Varies with Age of Disease Onset in Huntington Disease

\section{Contact:}

Allison Daley, MS, MPH

Research Genetic Counselor

allison.daley@osumc.edu

614-688-8672

Department of Neurology

2006 Kenny Road

Columbus, OH 43221

United States

M. Ranganathan, J.A. Race, D.C. Allain, S.K. Kostyk, A.M. Daley

The Ohio State University, Columbus, OH, USA (all authors)

Background: The Huntington disease (HD) phenotype is associated with changes in motor, behavioral, and cognitive function. The relationship between behavioral symptoms and age of onset (AOO) of clinical symptoms in HD has not been extensively explored.

Objective: This study aims to characterize the behavioral manifestations of $\mathrm{HD}$ in relationship to $\mathrm{AOO}$ of $\mathrm{HD}$ symptom presentation.

Methods: Data from 4469 individuals with manifest-HD were obtained from the Enroll-HD database. The odds of behavioral symptoms at disease presentation in individuals with earlier adult onset $(\mathrm{AOO}<30$ years; $n=479$ ), mid-adult-onset HD (AOO between 30 and 59 years; $n=3478$ ), and later adult-onset HD (AOO > 59 years; $n=512$ ) were compared. Problem behavior assessment results were used to gauge symptom frequency and severity. Descriptive statistics, $\chi^{2}$ tests, and multinomial logistic regression models were utilized for analysis.

Results: In the earlier adult onset cohort, 26\% $(n=126)$ of individuals had behavioral symptoms as the presenting symptom compared with $19 \%$ $(n=678)$ of individuals with mid-adult onset and $11 \%(n=56)$ of individuals with late-adult onset $(p<.0001)$. A one-year increase in AOO was associated with a $5.6 \%$ decrease in the odds of behavioral symptoms being the presenting symptom at disease onset $(p<0.0001)$. A 1 -year increase in $\mathrm{AOO}$ was associated with a $5.5 \%$ decrease in the odds of presentation with severe behavioral symptom of any type, particularly disorientation, delusions, and obsessive-compulsive disorder. There was no statistically significant relationship between $\mathrm{AOO}$ and risk of severe depression, suicidal ideation, or hallucinations.

Conclusions: Individuals with earlier onset HD may be more likely to present with behavioral symptoms, and particularly more severe behavioral symptoms than later-onset individuals. A better understanding of the relationship between $\mathrm{AOO}$ and behavioral phenotypes in HD will be helpful in the development of therapies to treat symptom-specific disease presentations. The observations in this study offer insight into future avenues of research.

\section{9}

Exploring the Relationship Between Brain Atrophy and Clinical Symptoms in Huntington Disease

\section{Contact:}

Gabriela Delevati Colpo

Postdoctoral research fellow

gabicolpo@gmail.com

713-321-0908

2221 W. Dallas St Apt 280

Houston, TX 77019

United States
Gabriela D. Colpo ${ }^{1}$, Natalia P. Rocha ${ }^{1,2}$, Leigh B. Latham ${ }^{2,3}$, Odelin Charron $^{3}$, George Papadimitropoulos ${ }^{3}$, Leorah Freeman ${ }^{3}$, Erin Furr Stimming $^{2,3}$, Antonio L. Teixeira ${ }^{1}$

${ }^{1}$ Department of Psychiatry and Behavioral Sciences, McGovern Medical School, The University of Texas Health Science Center at Houston (UTHealth), Houston, TX, USA; ${ }^{2}$ HDSA Center of Excellence at University of Texas Health Science Center at Houston, Houston, TX, USA; ${ }^{3}$ Department of Neurology, McGovern Medical School, The University of Texas Health Science Center at Houston, Houston, TX, USA

Rationale: Huntington disease (HD) is pathologically characterized by striatal atrophy. However, other regions, such as the globus pallidus, thalamus, and hippocampus also undergo degeneration. Less attention has been paid to these structures and their clinical relevance. Motor and nonmotor symptoms related to HD progression might be associated with atrophy of different brain regions.

Objective: This study aims to determine whether brain regional volumes are associated with HD clinical symptoms.

Methods: Eighteen patients with genetic diagnosis of HD (11 manifest $(48.6 \pm 10.9$ years; $3 \mathrm{M} / 8 \mathrm{~F})$, seven premanifest $\mathrm{HD}$ gene carriers $(47.3 \pm$ 9.4 years; $2 \mathrm{M} / 5 \mathrm{~F})$ ), and thirteen controls $(32.4 \pm 2.4$ years; $7 \mathrm{M} / 6 \mathrm{~F})$ underwent a brain magnetic resonance imaging (MRI) at $3 \mathrm{~T}$ with 3DMPRAGE for volumetric assessment. Segmentation of region of interest (ROIs) was performed using the Freesurfer v5.3.0. Symptoms were assessed using the Problem Behaviors Assessment (PBA), the Unified HD Rating Scale (UHDRS), the Symbol Digit Modalities Test (SDMT), the Verbal Fluency Test (VFT), and the Stroop interference test.

Results: Both premanifest HD gene carriers and manifest patients with HD presented a significant decrease in cortical $(p=0.001)$, gray matter $(p<0.001)$, and deep gray matter (DGM) $(p<0.001)$ volumes in comparison with controls. In premanifest HD gene carriers, lower DGM volumes, particularly in thalami and pallidi, were associated with higher CAP score (CAG age product - a proxy for cumulative disease burden) and worse scores in PBA depression and PBA irritability/aggression subscales. In manifest patients, atrophy of ROIs, such as putamen, pallidum, hippocampus and amygdala, was associated with worse motor symptoms as observed in the UHDRS and worse cognitive performance as evaluated by the SDMT, VFT, and Stroop.

Conclusions: Our preliminary results suggest that brain atrophy may be an early event in HD, consistent with previously published TRACK-HD findings. It is noteworthy that decreased volumes in subcortical structures are associated with behavioral symptoms in premanifest HD carriers and motor/cognitive symptoms in manifest patients with HD.

Funding: NPR is a Huntington's Disease Society of America (HDSA) fellowship recipient.

\section{0}

\section{Motor Speech Phenotypes in Huntington Disease}

Contact:

Sarah K. Diehl

Speech-language pathologist, doctoral student.

sarah.k.diehl@vanderbilt.edu

570-854-3371

2121 Fairfax Avenue

Unit \#16

Nashville, TN 37212

United States

Sarah K. Diehl, Antje Mefferd, Lin Ya-Chen, Michael de Riesthal, Daniel O. Claassen

Vanderbilt University Medical Center, Nashville, TN, USA (all authors) 
Patients with Huntington disease (HD) often experience dysarthria with disease progression. Motor speech deficits in HD have historically been described as hyperkinetic dysarthria due to chorea (Darley et al. 1969a), although this can vary tremendously, and more recent studies indicate that speakers with HD may not resemble one cohesive group but rather be grouped with speakers of other etiologies and dysarthria types (Lansford et al. 2014). This study aimed to identify distinct clusters of speech perceptual characteristics within a group of speakers with dysarthria secondary to Huntington disease.

A total of 48 participants with mild to moderate dysarthria due to HD completed routine motor speech screenings for this study. Six trained raters provided speech perceptual ratings for each speaker with HD. Salient features were examined for the group of 48 speakers as well as for clusters of speakers identified using an unsupervised k-means clustering approach. Subgroup relationship to clinical variables (e.g., disease duration, CAG repeats, medication).

Results indicate that speech perceptual characteristics did share some similarity with previous literature; however, notable differences were identified in speaking rate for the total group of speakers with HD. The cluster analysis revealed four subgroups of speakers, one of which was very mild, one with fast-speaking rate and a unique speech pattern, and two with slow-speaking rate and similar speech patterns that differed primarily in severity of dysarthria.

This study supports the existence of subgroups of speakers with varying salient features of dysarthria. The findings are discussed in relation to previous literature and clinical implications.

\section{1}

Small Molecule Modulator of Protein Disulfide Isomerase Attenuates Mutant Huntingtin Toxicity and Inhibits Endoplasmic Reticulum Stress in a Mouse Model of Huntington Disease

Contact:

Wenzhen Duan.

Associate Professor.

wduan2@jhmi.edu

410-502-2866

600 North Wolfe Street

CMSC 8-121

Baltimore, MD 21287

United States

Xiao Zhou ${ }^{1}$, Gang Li ${ }^{1,2}$, Anna Kaplan ${ }^{3}$, Michael M. Gaschler ${ }^{3}$, Xiaoyan Zhang $^{1}$, Zhipeng Hou ${ }^{1}$, Mali Jiang ${ }^{1}$, Roseann Zott ${ }^{4}$, Serge Cremers ${ }^{3,4}$, Brent R. Stockwell ${ }^{3}$, Wenzhen Duan

${ }^{1}$ Johns Hopkins University School of Medicine, Baltimore, MD, USA; ${ }^{2}$ Inner Mongolian Medical University, Huhehaote Shi, Neimenggu Zizhiqu, China; ${ }^{3}$ Columbia University, New York, NY, USA; ${ }^{4}$ Irving Institute for Clinical and Translational Research, Columbia University, New York, NY, USA

Huntington disease (HD) is caused by a cytosine-adenine-guanine trinucleotide repeat expansion in the huntingtin (HTT) gene encoding an elongated polyglutamine tract within the N-terminal of the huntingtin protein $(\mathrm{Htt})$ and leads to $\mathrm{Htt}$ misfolding, aberrant protein aggregation, and progressive appearance of disease symptoms. Chronic activation of endoplasmic reticulum (ER) stress by mutant $\mathrm{Htt}(\mathrm{mHtt})$ leads to cellular dysfunction and ultimately cell death. Protein disulfide isomerase (PDI) belongs to an ER chaperone family upregulated during ER stress that is responsible for the formation of disulfide bonds in proteins. Our previous studies demonstrated that $\mathrm{mHtt}$ caused PDI to accumulate at mitochondria-associated ER membranes and trigger cell death, and that modulating PDI activity or abundance using small molecules or RNAi protected cells again mHtt toxicity in cell and brain slice models of HD. In this study, we demonstrated that the PDI is upregulated in the HD human brain, in mouse models, and in cells expressing mHtt. Chronic administration of a reversible, brain-penetrable small-molecule PDI modulator, LOC14 $(20 \mathrm{mg} / \mathrm{kg})$, significantly improved motor function, attenuated brain atrophy, and extended survival in the N171-82Q HD mice. Moreover, LOC14 preserved medium spiny neuronal marker dopamine- and cyclic-AMP-regulated phosphoprotein of molecular weight 32,000 (DARPP32) levels in the striatum of HD mice. Mechanistic study revealed that LOC14 suppressed mHtt-induced ER stress, indicated by repressing the abnormally upregulated ER stress proteins in HD models. These findings suggest that LOC14 is promising to be further optimized for clinical trials of HD, and modulation of signaling pathways coping with ER stress may constitute an attractive approach to reduce $\mathrm{mHtt}$ toxicity and identify new therapeutic targets for treatment of HD.

\section{2}

MRI Reveals Brain Structural Atrophy, Metabolic Abnormality, and Disturbed Network Connectivity and the Correlation with Motor Impairment in Preclinical Study of Huntington Disease

\section{Contact:}

Wenzhen Duan

Associate Professor

wduan2@jhmi.edu

410-502-2866

600 North Wolfe Street

CMSC 8-121

Baltimore, MD 21287

United States

Jiangyang Zhang ${ }^{1,2}$, Susumu Mori ${ }^{1}$, Yihong Yang $^{3}$, Christopher A. Ross $^{4,5,6}$, Wenzhen Duan ${ }^{4,5,7}$

${ }^{1}$ Department of Radiology, Johns Hopkins University School of Medicine, Baltimore, MD; ${ }^{2}$ Department of Radiology, New York University School of Medicine, New York, NY, USA; ${ }^{3}$ Intramural Research Program, National Institute on Drug Abuse, Baltimore, MD, USA; ${ }^{4}$ Division of Neurobiology, Department of Psychiatry and Behavioral Sciences, Johns Hopkins University School of Medicine, Baltimore, MD, USA; ${ }^{5}$ Department of Neuroscience, Johns Hopkins University School of Medicine, Baltimore, MD, USA; ${ }^{6}$ Department of Pharmacology and Molecular Sciences, Johns Hopkins University School of Medicine, Baltimore, MD, USA; ${ }^{7}$ Program in Cellular and Molecular Medicine, Johns Hopkins University School of Medicine, Baltimore, MD, USA

Treatment of Huntington disease (HD) is likely to be most beneficial in the early, possibly premanifestation stage. The challenge is to determine the best time for intervention and evaluate putative neuroprotection in the absence of clinical symptoms. In this effort, several MRI modalities have been developing to monitor disease progression, including structural MRI, metabolic MRS, and functional MRI. We have characterized and validated longitudinal structural MRI measures as one of the most reliable and consistent objective measures in determining disease progression and treatment efficacy in HD mouse models. The brain metabolic changes were also detected significantly in HD mouse brain, reminiscent of some changes in human HD brain. Resting state functional MRI may represent an early biomarker in detecting neuronal dysfunction before degeneration. We recently examined whole-brain resting 
state fMRI connectivity and its correlation to impaired motor function in a mouse model of HD. HD mice demonstrated significantly disturbed functional connectivity, including decreased intra- and interstriatal connectivity, whereas increased connectivity between the striatum and anterior cingulate cortex compared with those in age-matched littermate control mice. Moreover, the reduced connectivity within striatum was associated with motor dysfunction. These findings demonstrated that multimodality MRI measures represent promising biomarkers not only to monitor disease onset and progression but also to evaluate the treatment efficacy in both presymptomatic and symptomatic trials of HD. Our results urge to consider employing MRI measures in clinical trials, particularly recently launched huntingtin lowering trials.

\section{3}

\section{Continuous Professional Development in HD: Learning to Support and Empower Families}

\section{Contact:}

John Eden

john.eden@hdscotland.org

07545582675

Business First

Linwood Point

Paisley PA1 2fb

United Kingdom

John Eden ${ }^{1}$, Louise McCabe ${ }^{2}$

${ }^{1}$ Scottish Huntington's Association, Linwood Point, Paisley PA1 2fb, UK; ${ }^{2}$ Stirling University, Stirling FK9 4LA, UK

In partnership, the Scottish Huntington's Association and Stirling University developed a Continuous Professional Development Module that delivers $200 \mathrm{~h}$ of blended learning in HD. The course takes the unique approach of integrating family stories and experiences with five models of care: person centered, family systems, biopsychosocial, personalization, and palliative care. The course focuses on the lived experience of HD and how these models can assist health and social care practitioners to support and enable families to enhance their quality of life. The module won the Scottish Council for Voluntary Organizations Award for best partnership in 2015, and we are currently working with Lancashire University to extend the course to practitioners in England and Wales.

\section{4}

\section{Centralized Sample Preparation and Analysis Considerations}

\section{Contact:}

Anka Ehrhardt

Director, Biofluids.

anka.ehrhardt@chdifoundation.org

609-945-9632

155 Village Boulevard

Suite 200

Princeton, NJ 08540

United States

Anka G. Ehrhardt; Cristina Sampaio

CHDI Foundation, 155 Village Boulevard, Suite200, Princeton, NJ 08540, USA (all authors)
Background and Aim: For select samples from Enroll-HD platform studies, standardized core services, including preparation of derived samples and commonly requested analysis, are being planned. The goal is to provide consistent quality, use the samples efficiently, and build a data collection providing larger context on the samples to create synergies, which will enable more efficient HD research from the samples.

Plans: The plans consist of two main parts: (1) Centralized and standardized core preparations when samples are requested (e.g., DNA and RNA isolation from PBMCs, cell-line generation (only when needed), etc.) and (2) discussions about an optimized core panel are ongoing (i.e., a set of analytes to be measured in all CSF samples, their matched plasma samples, and possibly additional samples). The panel is meant to cover commonly requested sample analysis (e.g., mHTT, HTT, NFL, tau, etc.).

Lay Summary: The analysis of biological samples from clinical studies yields essential data for studying HD. Centralized analysis of key samples can assure consistent high quality of data, as well as availability of these data to the entire HD research community. In this presentation, strategies for selecting the most important sample preparation and analysis for centralization are discussed.

\section{5}

Origin-HD: Genetic Modifiers of HTT CAG Intergenerational Repeat Instability in Male GDGECS

\section{Contact:}

Anka Ehrhardt

Director, Biofluids.

anka.ehrhardt@chdifoundation.org

609-945-9632

155 Village Boulevard

Suite 200

Princeton, NJ 08540

United States

Anka G. Ehrhardt ${ }^{1}$, Adam Ellenberger ${ }^{1}$, Ed Wild $^{2}$, Gail Owen ${ }^{2}$, Seung Kwak $^{1}$, Elena Pak ${ }^{1}$, Sherry Lifer ${ }^{1}$, Cristina Sampaio ${ }^{1}$

${ }^{1}$ CHDI Foundation, 155 Village Boulevard, Suite200, Princeton, NJ 08540, USA; ${ }^{2}$ UCL Institute of Neurology, Box 104, National Hospital for Neurology and Neurosurgery, Queen Square, London, WC1N 3BG, UK

Background: Earlier disease onset in subsequent generations, called anticipation, has been observed in HD families and is attributed to an increased cytosine-adenine-guanine (CAG)-repeat length in the huntingtin gene. CAG-repeat length mutations are referred to as "repeat instability," and genome-wide association studies suggest that genomic variants function as genetic modifiers of disease onset, for example, by affecting DNA repair mechanisms.

Aims: Origin-HD aims to investigate CAG-repeat instability in germline and somatic cells and evaluate for correlations with putative genetic modifiers. Understanding the mechanisms affecting repeat instability may yield testable targets for future interventions.

Study Design: Starting Q2 2019, over 1000 male HDGECs (ages 18-55) will be recruited from Enroll-HD participants over about 2 years, approximately balanced between premanifest and manifest disease. Allele and genotype frequency for each prespecified variant of interest will be assessed after about 500 participants have been recruited. If the predicted detectable effect size is $d>0.35$ in the final sample of 1000 participants, a recruitment-by-genotype recruitment approach can be adopted in parallel to the ongoing recruitment. The repeat instability in DNA from sperm and blood of study participants will be analyzed, and genetic modifier variants will be determined. 
26

\section{Biosample Access}

\section{Contact:}

Anka Ehrhardt

Director, Biofluids

anka.ehrhardt@chdifoundation.org

609-945-9632

155 Village Boulevard

Suite 200

Princeton, NJ 08540

United States

\section{Anka G. Ehrhardt, Dipinder Kaur}

CHDI Foundation, 155 Village Boulevard, Suite200, Princeton, NJ 08540, USA (all authors)

Background and Aim: Biosample collection is essential to developing therapeutics for Huntington disease. Biosample collections are intended to accelerate progress toward the development of therapeutics that will benefit HD-affected individuals.

Available Material and Access: Toward that goal, the longitudinal, code-protected clinical data, and renewable and nonrenewable biosamples collected from Enroll-HD, Registry-HD, Track-HD, TrackON, HDClarity participants are made available to any interested researcher working at a recognized research institution through a straightforward qualification process. The wide availability of these resources aims to energize HD research and encourage a broad variety of ideas and projects. Provided that participants have consented to biobanking, their biological samples collected at baseline and each follow-up visit are used to derive nonrenewable and renewable materials made available in aliquots.

\section{7}

\section{Enroll-HD Plasma Collection}

\section{Contact:}

Anka Ehrhardt

Director, Biofluids

anka.ehrhardt@chdifoundation.org

609-945-9632

155 Village Boulevard

Suite 200

Princeton, NJ 08540

United States

Mette Gilling ${ }^{1}$, Anka G. Ehrhardt ${ }^{2}$, Dipinder Kaur ${ }^{2}$, Jennifer Ware ${ }^{2}$, Olivia Handley ${ }^{1}$, Katrin Barth ${ }^{1}$, Shilpa Deshpande ${ }^{2}$, Sherry Lifer ${ }^{2}$, Selene Capodarca ${ }^{1}$, Ruth Fullam ${ }^{1}$, Travis Price ${ }^{2}$, Cristina Sampaio ${ }^{2}$, Eileen Neacy ${ }^{2}$, and the Core Enroll-HD PM Team

${ }^{1}$ The European Huntington's Disease Network, Enroll-HD Platform; ${ }^{2}$ CHDI Foundation Princeton, NJ 08540, USA

Increasing demand for high-quality plasma samples has led to a change in the Enroll-HD biosample collection. In addition to PBMCs from late 2018, EDTA plasma samples will be collected from select sites. The goal is to collect longitudinal EDTA plasma samples annually from 2000 to 3000 HDGECs at all disease stages, with a focus on premanifest and early-stage participants, plus another $10-15 \%$ of EDTA plasma samples from genenegative family members and family-control participants.

The current Enroll-HD study protocol and informed consent forms already include plasma collection, but a minor site agreement change will be necessary to allow for an additional extended procedure payment to sites. Enroll-HD sites will be identified according to eligibility criteria (including having trained staff to collect and process the samples, a $4{ }^{\circ} \mathrm{C}$ centrifuge, and $\mathrm{a}-80{ }^{\circ} \mathrm{C}$ freezer to store aliquoted samples until shipment) and invited to participate in the plasma collection.

\section{8}

\section{HD Research Data Access}

Contact:

Anka Ehrhardt

Director, Biofluids

anka.ehrhardt@chdifoundation.org

609-945-9632

155 Village Boulevard

Suite 200

Princeton, NJ 08540

United States

Mette Gilling ${ }^{1}$, Darren Freeman ${ }^{2}$, Eileen Neacy ${ }^{2}$

${ }^{1}$ The European Huntington's Disease Network, Enroll-HD Platform ${ }^{2}$ CHDI Foundation, Princeton, NJ 08540, USA

Background: During the era of large observational datasets like Registry, COHORT, and Enroll-HD, multiple additional Huntington disease (HD) studies have also contributed not only clinical data but also molecular profiles of muscle and fat tissue, genome-wide SNP associations, and different types of imaging datasets to the $\mathrm{HD}$ research community. In addition, many other studies are under way. The combined longitudinal or cross-sectional datasets offer a unique platform for further research studies to be conducted.

Aim: This study aims to make Huntington disease research data as easily accessible as possible within the limits of the informed consent and data use agreements.

Method: The purpose of this study is to create one application process across as many HD studies/datasets as possible.

\section{9}

\section{HDYO Feedback Project}

Contact:

Matt Ellison

Project Coordinator

matt@hdyo.org

07821799554

28 Millais Close

Bedworth CV12 8TH

United Kingdom

Matthew Ellison

HDYO, England.

HDYO intends to evaluate the impact of HDYO on young people and families, from the perspective and experiences of young people and families globally. HDYO is a charity that supports young people impacted by HD globally and has been around for 6 years, so we wanted to measure what impact we have had on young people by asking them to provide their feedback (positive and negative) on HDYO. This is being done through video interviews on Skype, and we are gathering all feedback from as many young people as possible. Feedback so far from young people I have interviewed has been $100 \%$ positive of their experiences of HDYO, with many emotional thank yous for helping them through tough times in their 
lives. Once all videos are done, we will produce one final video to highlight the impact of HDYO on young people and their families, as well as go through the qualitative data and review our impact, as we also hope to shape our decisions moving forward based partly on what young people think of us and what we currently do for them. The final video will be available to view at booths at $\mathrm{HD}$ events and on our YouTube channel.

30

Co-occurrence of Amyotrophic Lateral Sclerosis and Huntington Disease-a Systematic Review

\section{Contact:}

Wai Lun Alan Fung

Faculty Member, Dept of Psychiatry and Neuroscience Program, University of Toronto.

alan.fung.a@utoronto.ca

416-756-6322

4001 Leslie Street, 3SE

Genetics Program

Toronto, ON M2K 1E1

Canada

Wai Lun Alan Fung ${ }^{1,2}$, Harrison Mah ${ }^{1}$, Clare Gibbons ${ }^{1,3}$

${ }^{1}$ Multidisciplinary Huntington Disease Clinic, North York General Hospital, Toronto, Ontario, Canada; ${ }^{2}$ Department of Psychiatry, University of Toronto, Toronto, Ontario, Canada; ${ }^{3}$ Department of Molecular Genetics, University of Toronto, Toronto, Ontario, Canada

Background: Whereas Huntington disease (HD) and amyotrophic lateral sclerosis (ALS) are clinically distinct neurodegenerative disorders, there have been reports of the co-occurrence of the two conditions in single individuals - though the evidence to date has not been systematically summarized.

Aims: This study aims to (1) systematically review the literature on the co-occurrence of HD and ALS in single individuals; (2) to examine the common features among the cases of individuals with HD and ALS; and (3) to determine if the two disorders occurred together coincidentally or if they are causally related through an underlying mechanism.

Methods: A systematic review of all the literature on the cooccurrence of HD and ALS in single individuals - including case reports, observational studies, clinical trials, as well as mechanistic studies-until 20 July 2018 was performed, and descriptive analyses were conducted.

Results: Our searches have revealed 15 cases of individuals with confirmed diagnoses of both HD and ALS reported in the literature to date, and at least 5 other probable cases of HD and ALS. Among these 15 confirmed cases, the majority (13/15 cases) had an age of onset of HD symptoms between 40 and 72 years. These cases had 39-47 CAG repeats in the huntingtin gene. More than half of these cases (9/15) had a diagnosis for ALS ranging from several months to years after the diagnosis of HD. There have been no observational studies or clinical trials on the cooccurrence of HD and ALS to date. Two mechanistic studies on the cooccurrence of HD and ALS were identified.

Conclusion: The number of reported cases on the co-occurrence of HD and ALS remains very low, rendering it highly challenging to conduct observational studies or clinical trials. Nonetheless, there are some common features among the reported cases, and with some mechanisms of such co-occurrence proposed.

31

Nonpharmacological Interventions Targeting Psychiatric Manifestations in Huntington Disease Patients: a Scoping Review

\section{Contact:}

Wai Lun Alan Fung

Faculty Member, Dept of Psychiatry and Neuroscience Program, University of Toronto

alan.fung.a@utoronto.ca

416-756-6322

4001 Leslie Street, 3SE

Genetics Program

Toronto, ON M2K 1E1

Canada

Wai Lun Alan Fung ${ }^{1,2}$, Jeremy Lau ${ }^{3}$

${ }^{1}$ Multidisciplinary Huntington Disease Clinic, North York General Hospital, Toronto, Ontario, Canada; ${ }^{2}$ Department of Psychiatry, University of Toronto, Toronto, Ontario, Canada; ${ }^{3}$ Royal College of Surgeons in Ireland, Dublin, Ireland

Background: In addition to motor and cognitive symptoms, Huntington disease (HD) patients may also suffer from psychiatric symptoms such as depression, apathy, and irritability. Although various medications have been utilized, nonpharmacological interventions have also been used in treating these symptoms. However, little is known about their efficacy, and the evidence to date has not been systematically summarized.

Aims: This study aims to systematically review and evaluate the available literature regarding the efficacy and effectiveness of nonpharmacological interventions in treating the psychiatric manifestations associated with HD. Methods: A five-stage scoping review methodology according to Arksey and O'Malley (2005) was used to systematically identify and select relevant articles. These articles are summarized in narrative form.

Results: Our initial search yielded 4796 articles, of which 16 articles were selected and analyzed. A significant number of these articles were case studies $(n=5)$ and pilot studies $(n=3)$. The nonpharmacological treatments addressed by these studies included behavioral therapy, remotivation therapy, gardening, hypnosis, mindfulness-based cognitive therapy, music therapy, multisensory environment, psychosocial intervention, multimodality treatment, and exercise program.

Conclusions: Although nonpharmacological treatments were proposed to have positive effects, including reduction in anxiety, amelioration of depression, and increased quality of life, at present, the studies regarding this topic lack quality methodologies. Future studies should be done on a larger scale with psychiatric manifestations as primary outcomes and should explicitly account for the use of pharmacologic treatments within their methodology.

\section{2}

\section{Mutant Huntingtin Impairs Mitochondrial DNA Repair and Transcription}

\section{Contact:}

Rui Gao

Senior Research Associate

rugao@utmb.edu

409-774-4559

301 University Boulevard

Research Building 17, Room 4.500

Galveston, TX 77555

United States

Rui Gao ${ }^{1}$, Jeffrey Snowden ${ }^{1}$, Anirban Chakraborty ${ }^{2}$, Kara L. Gordon ${ }^{3}$, Nan Zhang ${ }^{4}$, Tetsuo Ashizawa ${ }^{4}$, Albert R. La Spada ${ }^{3}$, Tapas K. Hazra ${ }^{2}$, Partha S. Sarkar ${ }^{1}$ 
${ }^{1}$ Department of Neurology, Neuroscience, Cell Biology, and Anatomy, University of Texas Medical Branch, Galveston, TX, USA; ${ }^{2}$ Department of Internal Medicine, University of Texas Medical Branch, Galveston, TX, USA; ${ }^{3}$ Duke University School of Medicine, Departments of Biology, Duke University School of Medicine, USA; ${ }^{4}$ Department of Neurology, Houston Methodist Research Institute, Houston, TX, USA

The pathogenesis of Huntington disease (HD) can be attributed to the expansion of the polyQ tract in huntingtin (HTT). Compromised mitochondrial DNA (mtDNA) integrity and energetics are major pathogenic factors driving energy dyshomeostasis, neurotoxicity, and neurological decline in HD. However, the mechanism as to how polyQ expansions in HTT could cause mitochondrial dysfunction remains unknown. We report here that the wild-type HTT forms a mitochondrial transcription-coupled DNA repair (Mt-TCR) complex with mitochondrial RNA polymerase (POLRMT), mitochondrial transcription factors (TFAM, TFB1M, and TFM2M), and the DNA repair protein polynucleotide 5 '-kinase 3 '-phophatase (PNKP) and atxin-3 (ATXN3). We detected the presence of this complex through co-immunoprecipitation and confirmed its association with the mitochondrial genome via chromatin-immunoprecipitation. We have found that this novel complex plays a key role in maintaining mitochondrial genome integrity and function by repairing mitochondrial DNA during its transcription. The mutant HTT with expanded polyQ tracts, however, abrogated PNKP's 3'-phophatase activity, resulting in persistent accumulation of mitochondrial DNA strand breaks, thus leading to reduced transcript expression and impaired mitochondrial function. Overexpression of PNKP in the mutant cells dramatically improves mitochondrial integrity and physiology and reduces cell toxicity. Our findings establish a novel function for HTT, ATXN3, and PNKP in mitochondrial DNA maintenance and demonstrate how polyQ expansion in mHTT triggers signaling that degrades the structural and functional integrity of mitochondria, adversely affecting $\mathrm{mt}$ physiology and energetics in HD.

33

\section{Battling HD in Arabia}

\section{Contact:}

Hamda Al Hajri

al_hajriah@hotmail.com

96896150133

P.O 1842

P.C 114

Muscat 114

Oman

Hamda Al Hajri ${ }^{1}$, supported by Allison Gray Etheridge ${ }^{2}$

${ }^{1}$ Muscat, Oman ${ }^{2}$ Virginia, USA, but living in Oman

It has been a subject of shame talking about Huntington disease (HD) in my family and in Arabian society. It has been unrecognized and ignored. $\mathrm{HD}$, because of its nature, does not only affect the person diagnosed but everyone around them as well, family members and close friends. And because of the lack of recognition and support here, HD isolates those affected, forcing them to walk through it alone.

So, I would like to take this opportunity to put a spotlight on HD in my conservative Arabian society. I want to share how I have been learning to take sorrow and suffering and dance with them through this battle. I would like to share with you the story of HD in a place where illness is a source of shame and rejection, and suffering is not confronted, yet lived, in every day. I would like to share how I have been dancing through it all.
I am writing to you because I want a change. I am screaming for help. My dad and my sister passed away from HD. Two of my other sisters are diagnosed, and my brother is possibly showing symptoms. My aunts and my cousins as well are affected. More than 30 people in my family have been diagnosed, are showing symptoms, or at risk for developing HD. Family marriages are still continuing with no genetic checkups, and more and more kids are being born into the family.

It is time to bring awareness and break these habits. My dream is to be accepted into the HD global community and promote change and action in my society. My hope is that those who are affected by this disease may have a better quality of life here and the chance to have a voice. I want my family to know that we are not alone.

\section{4}

\section{The Visual System in Huntington Disease}

\section{Contact:}

Ali Hamedani

Physician

ali.hamedani@uphs.upenn.edu

919-426-3595

3400 Spruce St.

3 W. Gates Bldg.

Philadelphia, PA 19104

United States

Ali G. Hamedani, Tanya Bardakjian, and Pedro Gonzalez-Alegre

University of Pennsylvania, Philadelphia, PA, USA (all authors)

Introduction: Saccadic eye movement abnormalities are among the earliest manifestations of Huntington disease (HD) but are difficult to quantify at the bedside, and afferent visual pathway involvement in HD is poorly characterized. Here, we developed and evaluated a novel standardized protocol to assess eye movement and visual function in HD.

Methods: Participants with manifest HD $(n=15)$ and healthy controls $(n=13)$ performed the King-Devick test, a timed test of rapid-number naming that relies heavily on saccadic eye movement. Binocular highand low-contrast (2.5 and $1.25 \%$ ) acuity was measured using Sloan lowcontrast letter charts, and pupillometric recordings were made using a handheld NeurOptics PLR-3000 pupillometer. The NEI VFQ-25 questionnaire with ten-item neuro-ophthalmic supplement was also completed. United Huntington's Disease Rating Scale (UHDRS) motor score and other clinical and demographic variables were collected. Comparisons between manifest $\mathrm{HD}$ and controls were performed using linear regression adjusted for age and sex.

Results: Mean King-Devick reading time was $106.3 \mathrm{~s}$ in manifest HD and $51.3 \mathrm{~s}$ in controls $(p=0.00)$. Among manifest HD subjects, KingDevick time tended to be higher at higher UHDRS oculomotor, nonoculomotor, and total motor scores and at higher CAG repeat lengths, but these were not statistically significant. In unadjusted analyses, binocular high contrast acuity was seven letters (one Snellen line equivalent) lower in manifest HD than controls $(p=0.043)$, and this effect was similar at low-contrast acuity, but these associations were not statistically significant after adjusting for age. There were no differences in pupillary size, latency, velocity, or dilatation between manifest HD and controls. Individuals with manifest HD were reported to have poorer peripheral vision than controls $(p=0.011)$, but there were no differences in overall self-reported general health or vision.

Conclusion: HD is associated with abnormal performance on the King-Devick test of rapid number naming and poorer self-reported peripheral vision. Analysis of contrast acuity and other measures were limited by sample size and will be further assessed in this ongoing study. 
35

\section{Enroll-HD Lite}

\section{Contact:}

Olivia Handley, MD

olivia.handley@enroll-hd.org

07773469709

School of Biosciences

Cardiff CF10 3US

United Kingdom

Enroll-HD Platform

CHDI Foundation Inc., 350 Seventh Ave, Suite 200, New York, NY, USA

Enroll-HD Lite is a longitudinal, observational study of moderate-to-latestage HD participants. Participants will have previously participated in the Enroll-HD study. Enroll-HD Lite includes a lean assessment battery designed to continue to follow Enroll-HD study participants, offering a reduced visit burden while collecting critical milestones of disease progression. Baseline study assessments will take place at the study site. Annual follow-up visits may take place either at the study site or via a phone contact visit. There will be no biosample collection. Eligible participants will include moderate-tolate-stage HD gene expansion carriers and companion control participants. The study will follow a phased rollout plan, with the first wave expected to recruit between 500 and 800 participants from $\sim 15$ Enroll-HD sites. Once the initial phase has been completed, an interim analysis of the Enroll-HD Lite dataset will inform the overall scope of Enroll-HD Lite in terms of study population size and global reach.

\section{6}

\section{The (Scottish) National Care Framework for Huntington's Disease}

\section{Contact:}

Alistair Haw

National Care Framework Lead

alistair.haw@hdscotland.org

07736457247

Scottish Huntington's Association

Business First, Linwood Point

Paisley PA1 2FB

United Kingdom

\section{Alistair Haw}

Scottish Huntington's Association, Paisley, Renfrewshire, UK

Background: The Scottish Government commissioned the Scottish Huntington's Association to develop a National Care Framework for HD, followed by localized versions for each Scottish health region.

Aims: This study aims to develop a measurable, integrated health and social care model that guides providers and empowers HD families. The online format allows the framework to evolve over time to prevent it from becoming obsolete.

Method/Techniques: The framework was developed by an expert group representing families, psychiatry, psychology, neurology, neuropsychology, genetics, rehabilitation, dentistry, GPs, speech and language therapy, dietetics, physiotherapy, occupational therapy, care homes, palliative care, social work, and academia, an then subject to a full national consultation.

Results/Outcome: The National Care Framework has been backed by the Scottish Government and launched by the Health Secretary in the Scottish Parliament. Localized versions have been published in three health regions, with three more ready to be published shortly. Baseline surveys of staff and families have been conducted in each area, which will be repeated in 3 years' time to determine the impact of the frameworks.
Conclusions: The framework has been widely welcomed, both nationally and internationally, as an excellent model for HD and other conditions. Astri Arnesen, President of the European Huntington Association, says of the Framework: "The framework stands out as an invaluable resource on how to deal with HD. It is exactly what we need: not just information about $\mathrm{HD}$ but insight on how life with HD can be and how it can be managed. The framework manages to cover the immense complexity of the disease in a very structured and straightforward way. A wonderful tool-hereby warmly recommended. I hope it will be widely shared and used!" The Framework can be viewed at: care.hdscotland.org

\section{Cannabis Use in Huntington Disease}

\section{Contact:}

Trevor Hawkins, MD

Assistant Professor Neurology

trevor.hawkins@ucdenver.edu

440-554-7124

12631 East 17th Avenue Room 5113 Campus Box B185

Aurora, CO 80045

United States

Trevor Hawkins, Lauren Seeberger

University of Colorado Anschutz Medical Campus Aurora, CO, USA

Increasing numbers of patients anecdotally are using cannabis products to attempt to treat symptoms and different diseases. In particular, there has been a growing interest in treatment of neurological diseases. We sought to provide information about cannabis use among our Huntington disease (HD) clinic patient population. We used a self-administered anonymous survey on cannabis use completed by HD patients identified at the Huntington's Disease Center of Excellence at the University of Colorado Anschutz Medical Campus between 2017 and 2018. Overall, 22 patients responded to the survey. Of the responders, 8 out of the 22 responded saying that they used cannabis or marijuana. Half of our patients stated they used medical marijuana, whereas the other half used recreational. There was a roughly even distribution of the different methods/formulations with smoking the most common and edibles the least common. Eighty-six percent of patients who used cannabis used cannabidiol (CBD) products with a nearly even distribution of other subtypes or strains. They were asked which symptoms they are targeting with the treatment and the self-reported efficacy for the individual symptoms. The most common symptoms indicated for use for CBD were chorea, muscle spasms, and sleep. For the combined marijuana products, the most common indications were pain, depression, muscle spasms, and chorea. Overall, $80 \%$ of patients responded either somewhat or greatly improved for their symptoms, whereas two patients responded that their depression somewhat worsened. No significant side effects were reported. Our primary anonymous survey showed that a significant portion of HD patients in our clinic are using cannabis products to treat a myriad of symptoms. Overall, they report positive efficacy that may help to inform future clinical studies.

\section{8}

Platelets Contribute to Disease Pathology in Huntington Disease

Contact:

Denis Hélèna, MSc

PhD Student

helena14denis@gmail.com

$+15817778614$ 
833 Avenue Marguerite Bourgeoys

App. 301

Québec QC G1S3W7

Canada

Hélèna L. Denis ${ }^{1}$, Jérôme Lamontagne-Proulx ${ }^{1}$, Isabelle St-Amour ${ }^{1}$, Sarah L. Mason ${ }^{2}$, Jesse W. Rowley ${ }^{3}$, Nathalie Cloutier ${ }^{8}$, Marie-Ève Tremblay ${ }^{4}$, Antony T. Vincent ${ }^{5}$, Peter V. Gould ${ }^{6}$, Sylvain Chouinard ${ }^{7}$, Andrew S. Weyrich ${ }^{3}$, Matthew T. Rondina ${ }^{3}$, Roger A. Barker ${ }^{2}$, Eric Boilard $^{8}$, Francesca Cicchetti $^{1}$

${ }^{1}$ Centre de Recherche du CHU de Québec, Faculté de Médecine, Département de neurobiologie, Université Laval, Québec, QC, Canada; ${ }^{2}$ Cambridge Center for Brain Repair, University of Cambridge, Cambridge, UK; ${ }^{3}$ The Molecular Medicine Program and Department of Internal Medicine, University of Utah \& the George E. Wahlen VAMC GRECC, Salt Lake City, UT, USA; ${ }^{4}$ Centre de Recherche du CHU de Québec, Faculté de Médecine, Département de médecine moléculaire, Université Laval, Québec, QC, Canada; ${ }^{5}$ Institut de Biologie Intégrative et des Systèmes, Université Laval, Québec, QC, Canada; ${ }^{6}$ Service d'Anatomopathologie et de Cytologie du Centre Hospitalier Affilié Universitaire de Québec de l'Hôpital de l'Enfant-Jésus, Québec, QC, Canada; ${ }^{7}$ Centre Hospitalier Universitaire de Montréal-Hôtel Dieu, Movement Disorders Unit, CHU de Montréal, Québec, QC, Canada; ${ }^{8}$ Centre de Recherche du CHU de Québec, Faculté de Médecine, Département de microbiologie et immunologie, Université Laval, Québec, QC, Canada

Abstract: Huntington disease (HD) is caused by a mutation in the huntingtin gene $(\mathrm{Htt})$, which results in the production of an abnormal protein, mutant huntingtin $(\mathrm{mHtt})$, which is ubiquitously expressed and known to confer toxicity to multiple cell types. We have recently reported that HD brains are also characterized by vascular abnormalities, which include changes in blood vessel density/diameter as well as increased blood-brain barrier (BBB) leakage. Seeking to elucidate the origin of these vascular and BBB anomalies, we studied platelets that are known to play a role in maintaining the integrity of the vasculature and thrombotic pathways linked to this, given they surprisingly contain the highest concentration of $\mathrm{mHtt}$ of all blood cells. We assessed the functional status of platelets by performing ELISA, Western blot, and RNA-sequencing in a cohort of 71 patients with HD and 68 age- and sex-matched controls. We further performed hemostasis and platelet depletion tests in the R6/2 HD mouse model. Taken together, our findings indicate a modified transcriptome in HD, that the platelets in HD are dysfunctional with respect to the release of angiogenic factors, and in normal platelet functions including thrombosis, angiogenesis and vascular hemostasis. As such, platelets may play a critical hitherto unknown role in the pathogenesis of HD.

\section{9}

\section{Relationships Between Disease Characteristics and Caregiver Burden in Huntington Disease}

\section{Contact:}

Danielle C. Hergert

Neuropsychology Fellow

dblinkoff@mail.usf.edu

718-755-5042

7308 Wild Olive Ave. NE

Albuquerque, NM 87113

United States

Danielle C. Hergert ${ }^{1,2}$, Juan Sanchez-Ramos ${ }^{1}$, Cynthia R. Cimino ${ }^{1}$

${ }^{1}$ University of South Florida, Tampa, FL, USA ${ }^{2}$ New Mexico VA Health Care System, Albuquerque, NM, USA
Objective: Huntington disease (HD) is a genetic neurodegenerative condition that is characterized by cognitive, motor, and psychiatric symptoms. The purpose of this study was to explore which of these disease characteristics influence caregiver burden for HD caregivers.

Methods: Fifty patients diagnosed with manifest HD and 50 caregivers participated in the study at the University of South Florida. Average patient age was $52.3($ mean \pm S.D. $=11.3)$ and caregiver age was 52.2 (mean \pm S.D. $=14.4) ; 66 \%$ of caregivers were significant others, $12 \%$ parents, $6 \%$ children, and $12 \%$ were another type of relationship. Patients completed the Montreal Cognitive Assessment (MoCA) and were administered the Unified Huntington's Disease Rating Scale (UHDRS) motor exam and Apathy Evaluation Scale-Clinician version (AES-C). Caregivers completed the Caregiving Appraisal Scale.

Results: There were significant correlations between caregiver burden and caregiver age $(r=-0.4 ; p<0.01)$, MoCA score $(r=-0.378 ; p<0.01)$, UHDRS Motor score $(r=0.3 ; p<0.05)$, and clinician-rated apathy $(r=$ $0.43 ; p<0.01)$. There were no relationships between caregiver burden and patient age or disease duration. The significant variables were entered into a regression model. The model explained $46 \%$ of the variance, $R^{2}=$ $0.46, F(4,45)=9.75(p<0.001)$. The significant predictors were caregiver age $(\beta=-0.41 ; p<0.01)$, MoCA score $(\beta=-0.32 ; p<.05)$, and clinicianrated apathy $(\beta=2.3 ; p<0.05)$. Motor scores were no longer related to caregiver burden $(p=0.32)$.

Conclusions: There were significant relationships between caregiver burden, cognitive functioning, and clinician-rated apathy, but not motor scores. This suggests that possible interventions for caregivers may include providing education to caregivers on how to cope with patients' apathy and cognitive functioning. Caregiver age was associated with burden, with younger age being associated with increased burden when controlling for symptom severity. This has implications for this population in that HD typically has a younger age of onset than other neurodegenerative diseases. Therefore, caregivers may be younger and may be particularly at risk for experiencing caregiver burden.

\section{0}

Design of a Prospective, Longitudinal, Natural History Study in Huntington Disease

\section{Contact:}

Matt Gooding

matt.gooding@meditechmedia.com

+442073980557

Admiral House

76-78 Old Street

London EC1V 9AZ

United Kingdom

\section{Presenter:}

Gregory Hooper, MD

greg.hooper@roche.com

+442073980557

Roche Products Ltd.

Welwyn Garden City AL7 1TW

United Kingdom

Gregory Hooper ${ }^{1}$, Dylan Trundell ${ }^{1}$, Giuseppe Palermo ${ }^{2}$, Thomas $\mathrm{Kremer}^{2}$, Elsbeth Frick ${ }^{2}$, Lauren Boak ${ }^{2}$, Rachelle Doody ${ }^{2,3}$, Scott Schobel ${ }^{2}$

${ }^{1}$ Roche Products Ltd., Welwyn Garden City, UK; ${ }^{2}$ F. Hoffmann-La Roche Ltd., Basel, Switzerland; ${ }^{3}$ Genentech Inc., South San Francisco, CA, USA

Background: With the advent of huntingtin protein-lowering therapies and the ability to measure mutant huntingtin protein (mHTT) in 
cerebrospinal fluid (CSF), there is a need to better understand the potential of CSF mHTT as a biomarker for predicting and measuring Huntington disease (HD) progression. Observational studies, including HD-Clarity and UCL-CSF, aim to fill this knowledge gap, but CSF is only collected once every 1 or 2 years, respectively, leaving the shorterterm time course of CSF mHTT and associated change in biological and clinical outcome measures unexplored.

Aim: We are conducting a natural history study with a primary objective to determine the relationship between CSF levels of mHTT, clinical measures of HD progression, markers of neuronal injury, and brain atrophy over 15 months.

Methods/Results: This prospective, longitudinal, multisite natural history study will follow-up patients with early manifest HD (stage I or II). Clinical outcome measures will include composite Unified Huntington's Disease Rating Scale (cUHDRS), Total Functional Capacity (TFC), Total Motor Score (TMS), Symbol Digit Modalities Test (SDMT), and Stroop Word Reading (SWR) test. Other assessments include the measurement of CSF levels of mHTT, blood biomarkers, and brain imaging. The utility of clinical outcome measures collected via sensors in smartphones and wrist-worn wearables will be assessed. CSF will be collected at four time points, namely baseline, 3,9 , and 15 months.

Conclusion: This study will provide valuable information on the relationship between putative biomarkers, including mHTT, and clinical outcomes in HD. It is expected to aid interpretation of future clinical trials.

Funding Information: This study was funded by F. Hoffmann-La Roche.

Lay Summary: mHTT is a protein thought to cause HD and can be measured in collected spinal fluid. This study will provide valuable information on the relationship between levels of mHTT and a wide range of assessments that measure HD-related symptoms and their impact on daily function. Spinal fluid will be collected when patients begin the study and at 3, 9, and 15 months. Progression of the disease will be followed closely over 15 months and the results are expected to aid interpretation of clinical trials.

\section{1}

Predictive Testing for Huntington Disease Through Telemedicine: a Pilot Study

Contact:

Emily Housto

Study Coordinator

emily.houston@uvm.edu

802-656-8974

UVM Medical Center

1 South Prospect Street, Old Hall 2130

Burlington, VT 05401

United States

Emily Houston; James Boyd

The University of Vermont Medical Center, Burlington, VT, USA (all authors)

In North America, estimates indicate that nearly 30,000 individuals display clinical signs of Huntington disease (HD), and an additional 150,000 individuals are at risk of carrying the huntingtin gene mutation. While the age of requested predictive testing is trending lower, from an average of 40.4 to 36.4 years, the rate of testing utilization remains low. Multiple barriers may impact one's decision to pursue predictive testing, including travel distance, cost, and inflexibility of counseling requirements. The goal of this pilot project is to develop a telemedicine protocol that is inline with the recommended components of predictive testing put forth by the HDSA, and to determine the feasibility of this method of testing and counseling. The initial counseling session (genetic and supportive), disclosure of HD genetic test results, and the follow-up session will all be conducted via telemedicine in the participants' homes and will be compared with the standard in-person protocol in this nonrandomized, prospective cohort study. In-person and in-home telemedicine encounters will be compared by measures of satisfaction, feasibility, and psychological outcomes. We hypothesize that predictive testing through telemedicine could be an acceptable method of providing genetic results to those at risk of carrying the gene mutation for HD and that patients will be as satisfied with the telemedicine process as they are with the standard inperson process.

\section{2}

Impaired Oxygen Metabolism in the Brain During Visual Stimulation in Premanifest Huntington Disease Patients Detected by 3D-TRIP MRI at $7 \mathrm{~T}$

\section{Contact:}

Jun Hua

Assistant Professor

jhua@mri.jhu.edu

443-923-3848

Kirby Center, KKI, 707N Broadway

Baltimore, MD 21205

United States

Jun Hua ${ }^{1,2}$, Peter Klinkmueller ${ }^{1,2,3}$, Martin Kronenbuerger ${ }^{4,5}$, Xinyuan Miao $^{1,2}$, Russell L. Margolis ${ }^{4,5}$, Peter C. M. van Zijl ${ }^{1,2}$, Christopher A. $\operatorname{Ross}^{4,5,6}$

${ }^{1}$ F.M. Kirby Research Center for Functional Brain Imaging, Kennedy Krieger Institute, Baltimore, MD, USA; ${ }^{2}$ Neurosection, Division of MRI Research, Dept. of Radiology, Johns Hopkins University School of Medicine, Baltimore, MD, USA; ${ }^{3}$ Department of Electrical and Computer Engineering, Johns Hopkins University, Baltimore, MD, USA; ${ }^{4}$ Department of Neurology, Johns Hopkins University School of Medicine, Baltimore, MD, USA; ${ }^{5}$ Division of Neurobiology, Department of Psychiatry, Johns Hopkins University School of Medicine, Baltimore, MD, USA; ${ }^{6}$ Departments of Neuroscience and Pharmacology, Johns Hopkins University School of Medicine, Baltimore, MD, USA

Abstract: Metabolic and neurovascular abnormalities have been implicated in the pathophysiology of HD. Previously, we have shown altered baseline cerebral perfusion, as indicated by increased arteriolar-cerebralblood-volume (CBVa) in premanifest HD. We now examine dynamic responses in microvascular and metabolic parameters during functional stimulation in premanifest and manifest HD. The recently developed 3Dtriple-acquisition-after-inversion-preparation (3D-TRIP) MRI allows the measurement of signal responses in BOLD, cerebral-blood-flow (CBF), and cerebral-blood-volume (CBV) during functional stimulation in one single scan, from which the cerebral-metabolic-rate-of-oxygen $\left(\mathrm{CMRO}_{2}\right)$ response can be calculated. We studied $23 \mathrm{HD}$ subjects ( 15 premanifest, 8 early manifest) and 16 healthy controls. fMRI experiments were performed on a $7 \mathrm{~T}$ Philips scanner using 3D-TRIP during a flashing checkerboard paradigm compared wiht no stimulation (baseline). There was strikingly reduced $\mathrm{CMRO}_{2}$ response (" $\triangle \mathrm{CMRO}_{2}$ ") to visual stimulation in HD overall compared with control $(\mathrm{HD}, 6 \pm 4 \%$; control, $16 \pm 10 \%$; $p=0.001)$. Significant correlations were detected between estimated years-to-onset (YTO) and $\Delta \mathrm{CMRO}_{2}\left(R^{2}=0.49 ; p=0.001\right)$. All HD patients showed lower $\triangle \mathrm{CMRO}_{2}$ than the average control value, including three patients with $\mathrm{YTO}>20$ years. Some HD subjects showed completely absent $\triangle \mathrm{CMRO}_{2}$ response. The impaired $\triangle \mathrm{CMRO}_{2}$ response in HD may be linked to mitochondrial or other defects in cellular metabolism in HD. Metabolic deficits $\left(\Delta \mathrm{CMRO}_{2}\right)$ may occur earlier and present greater 
effect sizes than other fMRI changes, such as microvascular abnormalities $(\triangle \mathrm{CBV})$. Visual stimulation was used as a simple and robust paradigm to test our methodology. The detection of significant abnormality in $\triangle \mathrm{CMRO}_{2}$ in visual cortex in premanifest $\mathrm{HD}$ patients suggests that such effects may be present in other HD brain regions, tested with other sensory stimuli. These measures may help elucidate the metabolic defects in $\mathrm{HD}$ brain. $\mathrm{CMRO}_{2}$ measurement may prove a useful biomarker to track disease progression in manifest and premanifest HD, and we speculate that it may be responsive to therapeutic interventions.

Lay Summary: Progressive atrophy of striatum is an imaging hallmark for HD. However, brain structural atrophy changes slowly and may not be rapidly responsive to therapeutic interventions. We are therefore measuring functional brain changes using novel measures. Such abnormalities include perturbations in brain microvasculature and brain metabolic disturbances. Here, dynamic responses in metabolic rate of consumption of oxygen during functional stimulation was measured using an advanced MRI approach in premanifest HD subjects and healthy controls. Decreased oxygen consumption rate was observed in HD subjects compared with controls, which correlated with genetic status. The results elucidate the pathophysiology in HD in terms of metabolic deficiency and suggest the potential value of cerebral oxygen consumption as a biomarker for HD clinical trials.

\section{3}

\section{The Steps Tool: an Easy-to-Use Assessment Measure that Highlights Impairments Impacting Stair Safety}

\section{Contact:}

Deb Kegelmeyer

Clinical Professor

deb.kegelmeyer@osumc.edu

614-306-9899

453 West 10th Ave

Atwell Hall rm. 516

Columbus, $\mathrm{OH} 43210$

United States

Deb Kegelmeyer, Sandra Kostyk, David Kline, Katherine Ambrogi, Anne Kloos

The Ohio State University, Columbus, Ohio, USA (all authors)

Individuals with HD have progressive motor, cognitive, and behavioral impairments that contribute to difficulty with stair negotiation leading to falls on stairs. To assess stair safety, we developed a tool called the Step Test Evaluation of Performance on Stairs (STEPS) that allows systematic observation of movement and analysis of stair performance. The purpose of this study was to examine (1) the agreement of STEPS ratings between healthcare professionals who were experienced versus novice in movement analysis and (2) STEPS items with the lowest ratings, indicating areas to target fall prevention interventions.

Methods: Forty ambulatory individuals with a diagnosis of HD (mean age, 50.35) participated. Three observers (two experienced physical therapists (PTs) and one nurse with minimal experience in movement analysis) concurrently rated live performances of the STEPS.

Results: The nurse's average total STEPS ratings did not significantly differ from the experienced PTs' ratings with a difference of $-0.05(\mathrm{CI}=-0.53,0.43 ; p=0.83)$. Out of 16 items, the agreement between all raters was less than $75 \%$ for continuity of ascent $(69 \%)$ and descent $(72 \%)$, foot placement during ascent (74\%), and eccentric control of the knee during descent $(74 \%)$.
Individuals with HD were most likely to have difficulty during ascent/descent of stairs with foot placement and trunk stability. Additionally, many clients exhibited deficits in balance and eccentric control of the knee during descent.

Conclusions: Observation for continuity of movement has greater inter-rater variability than other STEPS items. Principal impairments that may negatively impact stair performance in HD are foot placement and control of descent along with trunk stability. Healthcare providers other than PTs can quickly be trained to use the STEPS tool reliably. Future areas to study would be whether therapy aimed at improving foot placement, trunk control, and eccentric quadriceps strength would improve safety on stairs.

\section{4}

Intravenous Transplantation of Human Dental Pulp Stem Cells Promotes AC Neurogenesis Recovery and Behavior Improvement in 3-Nitropropionic Acid Huntington Disease Rat Model

\section{Contact:}

Irina Kerkis

irina.kerkis@butantan.gov.br

+5511985446354

av. Vital Brasil, 1500

Sao Paulo 05503-900

Brazil

Cristiane Valverde Wenceslau ${ }^{1}$, Gustavo Sabino Lisboa ${ }^{1}$, Joyce Macedo da Silva ${ }^{2}$, Nicole Caroline Mambelli-Lisboa ${ }^{1}$, Monica Haddad ${ }^{3}$, Irina Kerkis ${ }^{4}$

${ }^{1}$ CellAvita Ltd., Valinhos, SP, Brazil; ${ }^{2}$ Azidus Brasil, Valinhos, SP, Brazil; ${ }^{3}$ Hospital das Clínicas da Faculdade de Medicina da Universidade de São Paulo, Sao Paulo, Brazil; ${ }^{4}$ Laboratório de Genética, Instituto Butantan, Sao Paulo, SP, Brazil

Mesenchymal stem cells (MSC) hold a great opportunity for neurodegenerative diseases acting through the expression of paracrine factors, thus providing neuroprotective therapy. These cells are isolated from postnatal tissues, such as bone marrow, adipose tissue, and umbilical cord. They were already employed in preclinical studies to treat Huntington's disease (HD). Human dental pulp stem cells (hDPSC) and neuronal cells share the same neural crest embryonic origin and express higher levels of neurotrophic factors than other MSC. This suggests they are more effective in the protection and repair of neural cells and tissues. To test the capacity of hDPSC to provide clinical benefits in HD, we reproduced a previously established 3-nitroporopionic acid acute treatment. Lewis rats $(n=124)$ were injected with 3-NP via intraperitoneal injection at a single dose of $20 \mathrm{mg} \mathrm{kg}^{-1}$ day $^{-1}$ for 4 days. The next day $(24 \mathrm{~h}$ after the 4 th $3-\mathrm{NP}$ injection), a single intravenous dose or three consecutive doses, one per month of $1 \times 10^{6}$ or $1 \times 10^{7} \mathrm{hDPSC}$ (approximately equivalent to $3.5 \times$ $10^{6}$ and $3.5 \times 10^{7} \mathrm{hDPSC} \mathrm{kg}^{-1}$, respectively), were administered. For the first time, we demonstrate that hDPSC derived from exfoliated deciduous teeth were able to pass through the blood-brain barrier (BBB) and to graft in the brain of 3-NP-treated rats following intravenous transplantation. The cells were safe after one versus three consecutive transplantations of hDPSC. Additionally, we analyzed the effect of these cells on neurogenesis and on functional recovery of the rats affected by HD. High endogenous BDNF, DARPP32, and D2R expression and neuronal regeneration were observed in the cortex, striatum, and caudate nucleus, 4 and 30 days after hDPSC transplantation, when compared with the placebo group. The group of animals that received hDPSC demonstrated accelerated recovery in motor function, which was already observed 4 days after cell transplantation. 
45

Equivalent Efficacy Versus Equal Effect Sizes in HD Clinical Trials

Contact:

Douglas Langben, $\mathrm{MD}, \mathrm{PhD}$

Professor

douglas-langbehn@uiowa.edu

319-384-4455

1-290 MEB

500 Newton Road

Iowa City, IA 52242

United States

Douglas R Langbehn, MD, $\mathrm{PhD}$

University of Iowa, Iowa City, Iowa, IA, USA

The potential for testing a putative Huntington disease (HD) treatment is clearly related to the cost and, therefore, the sample size of the required clinical trials. When we compare the hypothetical sample sizes needed for HD trials involving various potential outcomes (e.g., brain imaging, motor scores, cognitive scores, total functional capacity, CSF assays), we often, uncritically, make strong, qualitative assumptions about the relationship between outcome measures, underlying illness, and the effect of a therapy. Regulators, with good reason, may be less willing to take these assumptions for granted, thus they deserve explicit examination.

1. All candidate outcomes, if measured ideally, are equally appropriate indicators of illness severity.

2. The association between the outcome and underlying illness severity will not be disrupted by the therapy.

3. All candidate measures will show an equivalent response to therapy. Clinical trial sample sizes are determined by a hypothesized signal-tonoise ratio (effect size), in which the measured average response to treatment is the "signal" and other unpredictable variation in the outcome's change during the treatment trial is the "noise." The three above assumptions are equivalent to assuming that the treatment response signals for all measures would be both equal and equivalent indicators of efficacy. Comparisons are to be based only on the relative noisiness of these treatment signals. These assumptions become especially questionable when evaluations of competing outcomes require different statistical models. We illustrate with a comparison of motor score change versus diagnosis prevention in preHD trials.

It is not difficult to imagine scenarios in which these assumptions are untenable. Direct surgical manipulation of the brain may dissociate the relationship between efficacy and imaging. Another example is when a symptomatic treatment targets limited aspects of the disease. We urge explicit acknowledgment and discussion of these assumptions when HD experts and statisticians collaborate to compare trial design choices.

\section{6}

Partial Lowering of Total Huntingtin Levels to Treat Adults with Huntington Disease: Potential Benefits and Theoretical Risks from Human Studies and Animal Models

Contact:

Matt Gooding

Matt.Gooding@meditechmedia.com

+442073980557

Admiral House

76-78 Old Street

London EC1V 9AZ

United Kingdom
Presenter:

Blair R. Leavitt

bleavitt@cmmt.ubc.ca

+442073980557

Centre for Molecular Medicine and Therapeutics, Department of Medical

Genetics

University of British Columbia

Vancouver V6T 1Z4

Canada

Blair R. Leavitt ${ }^{1}$, Scott A. Schobel ${ }^{2}$, Holly Kordasiewicz ${ }^{3}$

${ }^{1}$ Centre for Molecular Medicine and Therapeutics, Department of Medical Genetics, University of British Columbia, Vancouver, BC, Canada; ${ }^{2}$ F. Hoffmann-La Roche Ltd., Basel, Switzerland; ${ }^{3}$ Ionis Pharmaceuticals Inc., Carlsbad, CA, USA

Background: RG6042 (formerly IONIS-HTTRx) is an antisense oligonucleotide (ASO) in development for Huntington disease (HD), which partially lowers levels of both mutant and wild-type huntingtin (mHTT and wtHTT). In a phase 1/2a study in adults with HD, RG6042 lowered levels of mHTT in cerebrospinal fluid with no safety signals. Nevertheless, theoretical risks of lowering wtHTT remain.

Aim: This study aims to review published clinical and in vivo studies involving reducing wtHTT levels.

Methods: A comprehensive review and summary of relevant articles from PubMed.

Results: People with reduced wtHTT levels due to a rare disruption in one HTT copy did not have HD or other abnormalities (one study). People with two mutant HTT copies (i.e., no wtHTT) developed normally until HD onset at a similar age to heterozygotes, with similar symptoms (four studies). No detrimental effects of partial wtHTT lowering were reported in normal adult animals (rodents, five studies; nonhuman primates, four studies) or HD rodent models (four studies). ASO-mediated mHTT lowering ameliorated disease in three HD mouse models, with similar benefits for total HTT (wtHTT plus mHTT) lowering (one study). In HD mouse models, wtHTT knockouts (1 study) and mice with $10 \%$ of normal wtHTT levels from conception (one study) had worse HD-like phenotypes than those with normal levels, but wtHTT overexpression did not alleviate phenotypes (two studies). Although HTT is essential for embryogenesis (four studies) and development (two studies), nearcomplete genetic ablation of wtHTT expression in adulthood had little/ no reported neurologic effects in mice (two studies).

Conclusion: Studies indicate that theoretical risks of partially lowering wtHTT in adults are low. Unlike gene silencing/editing, lowering with ASOs is transient and reversible, and the dose is titratable, allowing risk mitigation. As RG6042 is not mutation specific, it has potential to lower HTT levels in all adults with HD.

Funding Information: This study was funded by F. Hoffmann-La Roche.

Lay Summary: A new drug in development for HD, RG6042, works by lowering levels of a toxic version of a protein called huntingtin, but may also lower levels of the normal version of this protein. A search of existing scientific literature has shown that the safety risks of lowering normal huntingtin are minimal.

\section{7}

Digital, High-Frequency, Long-Term Monitoring of Motor and Nonmotor Symptoms in Huntington Disease Patients

Matt Gooding

Matt.Gooding@meditechmedia.com

+442073980557

Admiral House, 76-78 Old Street, London EC1V 9AZ, UK 


\section{Presenter:}

Florian Lipsmeier

florian.lipsmeier@roche.com

+442073980557

Roche Pharmaceutical Research and Early Development

Roche Innovation Center Basel

Basel 4070

Switzerland

Florian Lipsmeier ${ }^{1}$, Wei-Yi Cheng ${ }^{1}$, Detlef Wolf ${ }^{1}$, Yan-Ping Zhang ${ }^{1}$, Timothy Kilchenmann ${ }^{1}$, Atieh Bamdadian ${ }^{1}$, Anne Smith ${ }^{2}$, Edward Wild ${ }^{3}$, Scott Schobel ${ }^{4}$, Christian Czech $^{1}$, Christian Gossens ${ }^{1}$, Michael Lindemann ${ }^{1}$

${ }^{1}$ Roche Pharmaceutical Research and Early Development, Roche Innovation Center Basel, Basel, Switzerland; ${ }^{2}$ Ionis Pharmaceuticals Inc., Carlsbad, CA, USA; ${ }^{3}$ UCL Huntington's Disease Centre, Institute of Neurology, National Hospital for Neurology \& Neurosurgery, Queen Square, London, UK; ${ }^{4}$ F. Hoffmann-La Roche Ltd., Basel, Switzerland

Background: Smartphones, wearables, and other consumer technology have high-quality sensors that enable the objective, remote, continuous, and longitudinal measurement of disease symptoms. This approach has the potential to be used in future drug development and clinical practice. Aim: In this study, we are exploring the feasibility of using smartphones and wrist-worn wearables for high-frequency monitoring of motor and nonmotor Huntington's disease (HD) symptoms.

Method: Patients with HD in the ongoing ISIS 443139-CS2 clinical study (planned enrollment, $n=46$ ) receive a smartphone and wrist-worn wearable during screening. Using these tools, patients are asked to complete daily "active" tests at home that have been designed to measure a range of motor and nonmotor symptoms. Motor symptoms that are being tested include chorea and gait problems; nonmotor symptoms that are in focus include cognitive processing speed. In addition, patients are asked to carry the devices with them as they go about their daily activities (i.e., "passive monitoring"). The sensor data are then securely transferred via WIFI in real-time to Roche, where they are processed and analyzed for adherence and clinically meaningful sensor data features.

Results: Preliminary data uploaded during the first 8-week observational period in the study show that adherence is good: $\sim 85 \%$ of subjects completed the entire active test suite on three or more days each week.

Conclusion: It is feasible to measure motor and nonmotor function in patients with $\mathrm{HD}$ using smartphone-based assessments performed at home. This technology, including real-time data transfer and advanced analytics, provides an innovative way to learn more about patients' daily symptoms in the clinical trial setting. Data collection in additional patient cohorts will be an important next step to broaden experience with this approach.

Funding Information: This study is funded by Ionis Pharmaceuticals Inc. and F. Hoffmann-La Roche.

Lay Summary: A study is underway to determine if smartphones and other wrist-worn wearable technology can be used to measure Huntington disease symptoms in daily life using at-home tests and continuous movement tracking. The first results from the study show that most patients consistently completed the tests, and that this may be a useful way of monitoring patients' symptoms, supplementing clinic visits.

\section{8}

Enrichment Strategy in Early-to-Moderate Manifest Huntington Disease Based on CAG/Age Product (CAP) $>400$ Threshold

Contact:

Matt Gooding

Matt.Gooding@meditechmedia.com

+442073980557

Admiral House
76-78 Old Street

London EC1V 9AZ

United Kingdom

Presenter:

Jeffrey D. Long

jeffrey-long@uiowa.edu

+442073980557

Department of Psychiatry

Carver College of Medicine, University of Iowa

Iowa City, IA 52242

United States

Jeffrey D Long ${ }^{1,2}$, Bernhard Landwehrmeyer ${ }^{3}$, Giuseppe Palermo ${ }^{4}$, Scott Schobel $^{5}$, Sarah J. Tabrizi ${ }^{6}$

${ }^{1}$ Department of Psychiatry, Carver College of Medicine, University of Iowa, Iowa City, IA, USA; ${ }^{2}$ Department of Biostatistics, College of Public Health, University of Iowa, Iowa City, IA, USA; ${ }^{3}$ Department of Neurology, University of Ulm, Oberer Eselsberg 45, 89081, Ulm, Germany; ${ }^{4}$ Roche Pharma Research and Early Development, Roche Innovation Center Basel, Basel, Switzerland; ${ }^{5}$ F. Hoffman-La Roche Ltd., Basel, Switzerland; ${ }^{6}$ Huntington's Disease Research Centre, UCL Institute of Neurology, London, UK

Background: Huntington disease (HD) is a fatal, monogenic, neurodegenerative disease, which results in relentless progression of motor, cognitive, and behavioral domains, leading to overall functional decline. Clinical staging in HD is based on the Total Functional Capacity (TFC) scale, a subjective clinician-rated outcome, which does not uniformly predict the rate of disease progression. The CAG/Age Product (CAP) is an objective measure that combines an individual's CAG repeat length with age, providing an index of how long an individual has lived with his or her mutation burden. CAP has several forms and here we consider CAP = (age at study entry $) \times(\mathrm{CAG}-33.66)$ based upon the Iowa method.

Aim: This study aims to demonstrate the value of applying a CAP threshold as an inclusion criterion in a hypothetical clinical trial of early-tomoderate HD patients.

Method: Based on multiple datasets (TRACK-HD, ENROLL-HD, and REGISTRY), we applied generalized least squares (GLS) regression to model the change in the composite Unified Huntington's Disease Rating Scale (cUHDRS) and TFC over time and derived trajectories of decline in CAP subgroups, as well as signal-to-noise in a full versus a CAP-enriched population. Results: Use of a CAP $>400$ threshold results in a more reliable pattern of clinical progression on key endpoints such as functional loss as measured by the TFC scale or cUHDRS, while still including over $90 \%$ of the early HD population.

Conclusion: Selection of patients based on CAP $>400$ threshold is expected to result in a larger signal-to-noise ratio on clinical endpoints like TFC or cUHDRS and thus translate into a more efficient clinical trial requiring fewer participants.

Funding Information: This study was funded by F. Hoffmann-La Roche. Lay Summary: Testing a new investigational drug for a slowing of clinical progression in $\mathrm{HD}$ requires a patient population that will experience clinical progression by the end of the trial. Measuring disease progression with the Total Functional Capacity scale alone results in a variable rate of clinical progression. The CAG/Age Product (CAP) method, which uses a combination of CAG repeat length and age, to select patients, was found to be a more reliable way to predict a person's rate of clinical progression.

HD-POP: a Population-Based Study of Huntington Disease

Contact:

Clement Loy, MD 
Associate Professor

clement.loy@sydney.edu.au

+612-98456793

Hawkesbury Road

Westmead

Sydney NSW 2145

Australia

\section{C.T. Loy $^{1,2}$, S. Graham ${ }^{1}$, R. Trent ${ }^{2,3}$, E. McCusker ${ }^{1,2}$}

${ }^{1}$ Huntington Disease Service, Westmead Hospital, Sydney, Australia; ${ }^{2}$ The University of Sydney, Sydney Australia; ${ }^{3}$ Royal Prince Alfred Hospital, Sydney, Australia

Background/Aims: Observational studies in Huntington disease (HD) to date have been instrumental for clinical trial design. However, these volunteer-based studies may not reflect $\mathrm{HD}$ in the community and have sparse data on advanced HD. We aimed to assess the prevalence, clinical and social impact, and survival rate of $\mathrm{HD}$, through a population-based study. Methods: 1) Prevalence was measured using a case-finding survey of all neurologists, clinical geneticists, and psychiatrists at chronic psychiatric hospitals (census date, 1 Jan 2012), in the State of New South Wales (population, 7.4 million). Methodology was identical to our 1996 prevalence study. 2) Among people in the 2012 prevalence study living in Greater Sydney, 93\% were known to the Westmead HD Service (WHDS), forming the Greater Sydney Cohort (GSC, $N=270$ ). Clinical and social impact of HD at census were assessed through retrospective chart review. 3) Survival rate was calculated from follow-up of people with HD living in Greater Sydney from our 1996 study, who were known to WHDS (81\%).

Results: (1) Ninety-four percent of the 256 clinicians surveyed responded. Prevalence increased from 6.29 (1996) to 7.56/100,000 in $2012(p=0.012)$. Twenty-six percent of people were first diagnosed with $\mathrm{HD}$ at age $>=60$, and we will present age-specific prevalence. (2) For the population-based GSC cohort, we will present the distribution of CAGrepeat lengths, presenting symptoms and socioeconomic status. Sixtyeight percent of the cohort lived at home, and we will present the distribution of UHDRS motor scores, and prevalence of cognitive impairment (data available for $>=76 \%$ ). Thirty-two percent were in residential care. We will present age of admission and care needs in terms of mobility, feeding, activities of daily living, and communication (data available for $>=78 \%$ ). (3) Median survival was 15 years (IQR10-21).

Conclusion: We present a population-based "snapshot" of HD. With emerging disease-modifying therapies, this study will help inform health economics, service planning, and advocacy.

\section{0}

\section{Demonstration of Prion-Like Properties of Mutant Huntingtin Fibrils in In Vitro Paradigms}

\section{Contact:}

Maria Masnata

$\mathrm{PhD}$ student

marmasnata@gmail.com

$1(581) 991-2771$

804 rue Beauregard \#3

Quebec City, QC G1V 2 Y2

Canada

Maria Masnata ${ }^{1,2}$, Giacomo Sciacca ${ }^{1,2}$, Alexander Maxan ${ }^{1,2}$, Florian Lauruol $^{1,2}$, Hélena Denis ${ }^{1,2}$, Linda David ${ }^{1,2}$, Martine Saint-Pierre ${ }^{1,2}$, Jeffrey Kordower ${ }^{4}$, Luc Bousset ${ }^{3}$, Ronald Melki ${ }^{3}$, Melanie Alpaugh ${ }^{1,2}$, Francesca Cicchetti ${ }^{1,2}$

${ }^{1}$ Centre de Recherche du CHU de Québec (CHUQ), Ax Neurosciences, 2705 Boulevard Laurier, Québec, QC, G1V 4G2 Canada; ${ }^{2}$ Département de Psychiatrie \& Neurosciences, Université Laval, Québec, QC, G1K 0A6 Canada; ${ }^{3}$ Paris-Saclay Institute of Neuroscience, CNRS, Gif-surYvette, France; ${ }^{4}$ Department of Neurological Sciences, Rush University Medical Centre, Chicago, IL 60612, USA

In recent years, evidence has accumulated suggesting that mutant huntingtin protein $(\mathrm{mHtt})$ can spread into normal healthy tissue in a prion-like fashion. This theory, however, has remained controversial. To evaluate the capacity of $\mathrm{mHtt}$ to seed pathology in vitro, multiple cellular models were treated with exogenous human fibrillar huntingtin (Htt) (Q25) and $\mathrm{mHtt}(\mathrm{Q} 48)$ fragments. Human neuronal cells (induced pluripotent stem cell-derived GABA neurons (iGABA) and SH-SY5Y)), as well as human THP-1-derived macrophages, were incubated with synthetic $\mathrm{mHtt}$ fibrils for 2 to 5 days; in parallel, SHSY5Y cells alone were treated for 7 days in combination with Q19 Httcontaining conditioned media. Both synthetic fibrils were incorporated by all cell types, revealing a predominance of $\mathrm{mHtt}$ detected inside the cells in comparison with Htt in SH-SY5Y and THP1 macrophages. Once uptaken, $\mathrm{mHtt}$ fibrils induced an increase in cell death and dysfunction. In iGABA cells, despite no difference in $\mathrm{mHtt} / \mathrm{Htt}$ uptake ratio, $\mathrm{mHtt}$ fibril administration promoted toxicity measured as a change in neuronal morphology, specifically a shortening of primary neurites and a reduction of the number of neurons with secondary projection. In SH-SY5Y, an increase in the number of cleaved caspase $3^{+}$cells was detected when incubated with $\mathrm{mHtt}$. However, increased apoptotic THP-1 cells were detected just within the population of cells containing Q48 fibrils, suggesting different vulnerability to $\mathrm{mHtt}$ exposure between neuronal and non-neuronal cell lines. In association with the changes in cell vitality, differences in the staining pattern of wild-type endogenous Htt was also observed after incubation with fibrils in both THP-1 and SH-SY5Y cells. Furthermore, in SH-SY5Y cells, a difference in the pattern of exogenously administrated Q19 Htt, provoked by fibrillar $\mathrm{mHtt}$ coincubation, was as well established. These findings indicate that exogenous $\mathrm{mHtt}$ fibrils are toxic to multiple cell types and are capable of inducing prion-like changes in $\mathrm{Htt}$.

\section{1}

\section{Adeno-Associated Virus Mediated Delivery of Mutant Huntingtin in Mice and Primates to Model Huntington Disease}

\section{Contact:}

Alexander Maxan

alexander.maxan.1@ulaval.ca

418-262-9277

3062 Rue des Chatelets

apt 10

Québec QC G1V $3 Z 2$

Canada

A. Maxan ${ }^{1}$, G. Sciacca ${ }^{1}$, L. Breger ${ }^{2}$, B. Dehay ${ }^{2}$, Zhu Tao ${ }^{3}$, Zhang Ling ${ }^{3}$, Chuan Qin ${ }^{3}$, A. Oueslati ${ }^{1,4}$, E. Bezard ${ }^{2}$, F. Cicchetti ${ }^{1,5}$

${ }^{1}$ Centre de Recherche du CHU de Québec, Ax Neurosciences, Québec, QC G1V 4G2, Canada; ${ }^{2}$ Institut des maladies neurodégénératives, CNRS UMR 5293, Université de Bordeaux, Bordeaux, France; ${ }^{3}$ Institute of Laboratory Animal Sciences, China Academy of Medical Sciences, Beijing, China; ${ }^{4}$ Département de Médicine Moléculaire, Université Laval, Québec, QC G1K 0A6, Canada; ${ }^{5}$ Département de Psychiatrie \& Neurosciences, Université Laval, Québec, QC G1K 0A6, Canada

Objective: In recent years, publications have reported experimental evidence that part of the pathogenic process in a number of neurodegenerative disorders involves the spreading of abnormal proteins. However, in Huntington disease (HD), the classical dogma purports that the abnormal protein, mutant huntingtin ( $\mathrm{mHtt})$, causes cellular dysfunction through cell-autonomous processes. We have recently discovered $\mathrm{mHtt}$ 
aggregates within fetal striatal transplants in HD patients years after they were grafted, providing evidence of $\mathrm{mHtt}$ spread. The study presented here was designed to study routes of protein spread and attempt to model various aspects of the disease in mice and primates.

Methods: We performed three distinct protocols of adeno-associated virus (AAV) expressing green fluorescent protein (GFP) injections alone or coupled to Htt-Exon1 (19Q, 67Q, or 103Q) to deliver mHtt to the CNS of: (1) adult wild-type (WT) mice receiving a single injection intracortically $(n=40)$; (2) adult WT mice receiving multiple injections intracortically and intrastriatally $(n=36)$; and (3) adult WT primates receiving multiple injections intrastriatally $(n=5)$. All animals were subjected to motor behavioral testing and postmortem analyses. In single-injected mice, 2-photon microscopy was also conducted.

Results: Statistically significant changes were observed in motor behavior of mice and monkeys receiving multiple injections (open field, cylinder, clasping, and pole tests in mice, locomotion and object retrieval in primates), whereas those receiving a single injection showed statistically significant differences in early time points of some motor tests (pole test). Twophoton intravital imaging revealed uptake of GFP-tagged Exon1-103Q by blood vessels and $\mathrm{GFAP}^{+}$cells, supported by postmortem analysis. $\mathrm{GFP}^{+}$ aggregates were also seen in structures distant from the injection site.

Conclusion: Our preliminary results indicate that GFP-tagged mutant huntingtin protein can propagate intercellularly, providing a suitable model to help us to understand how this protein spreads and how we can target it for the development of efficient therapies.

52

\section{D-Serine Levels in Huntington Disease: a Cross-Sectional Exploratory Study}

\section{Contact:}

Andrew McGarry, MD

Associate Professor of Neurology

mcgarry-andrew@cooperhealth.edu

856-342-2445

3 Cooper Plaza

Suite 320

Camden, NJ 08103

United States

Andrew McGarry ${ }^{1}$, Basant Pradhan ${ }^{1}$, Trisha Niblack ${ }^{1}$, Cory Hackmyer ${ }^{1}$, Hamza Shaikh ${ }^{1}$, Irving Wainer ${ }^{2}$

${ }^{1}$ Cooper University Healthcare at Rowan University, Camden, NJ, USA; ${ }^{2}$ Mitchell Woods Pharmaceuticals, Shelton, CT, USA

Background: D-Serine (DSR), the most biologically active D-amino acid in the mammalian brain, plays a significant role in glutamate signaling, dendritic development, synaptic plasticity, and neuronal migration. It is a co-agonist of the NMDA receptor (NMDAR) by binding potently to the NMDA-associated glycine modulatory site (GMS). DSR is highly expressed in brain regions enriched with NMDAR, such as cortex, hippocampus, thalamus, hypothalamus, and amygdala. It is active at synaptic NMDAR, where the most common forms of plasticity, long-term potentiation, and depression are mediated. Strict regulation of NMDAR activity is also thought to be relevant to neurotoxicity. NMDA hyperactivity has been implicated in cell death for several neurodegenerative disorders; conversely, NMDAR hypoactivity promotes apoptosis and contributes to mild cognitive impairment (MCI) and psychosis/cognitive impairment in schizophrenia. Little is known about DSR biology in HD. It is not clear if DSR helps potentiate HD toxicity, can serve as a biomarker of onset or progression, or facilitates an adaptive response at synaptic NMDAR receptors that may be amenable to therapeutic amplification.

Methods: In this pilot study, we quantify DSR levels in plasma and CSF and correlate data to clinical severity at a single time point from different stages (HD1, HD2, HD3).
Results: As of 21 July 2018, 12 subjects of varying severity have successfully participated, with collection of clinicometric data, plasma, and CSF. Subjects 13 and 14 will participate in August 2018. Data will be analyzed in September 2018 for presentation.

Discussion: In a context in which no specific treatment exists for cognitive decline in HD, it is intriguing to consider that DSR may influence synaptic plasticity toward improved cognitive performance. Data will guide development of several projects, including a larger prospective study tracking DSR over longer periods and pilot projects aimed at testing DSR, alone or in combination with other molecules, as a therapeutic agent in HD.

\section{3}

Risk Factors for Suicidality in Huntington Disease in the Context of a Randomized Clinical Trial: an Analysis of the 2CARE Study

Contact:

Andrew McGarry, MD

Associate Professor of Neurology

mcgarry-andrew@ cooperhealth.edu

856-342-2445

3 Cooper Plaza

Suite 320

Camden, NJ 08103

United States

Andrew McGarry ${ }^{1}$, Michael P. McDermott ${ }^{2}$, Karl Kieburtz ${ }^{2}$, Wai Lun Alan Fung ${ }^{3}$, Elizabeth McCusker ${ }^{4}$, Elisabeth A. de Blieck ${ }^{2}$, Merit Cudkowicz ${ }^{5}$

${ }^{1}$ Cooper University Healthcare at Rowan University, Camden, NJ, USA; ${ }^{2}$ University of Rochester, Rochester, NY, USA; ${ }^{3}$ North York General Hospital 2, Toronto, ON, CA; ${ }^{4}$ Westmead Hospital, Westmead, NSW Australia; ${ }^{5}$ Harvard Medical School, Boston, MA, USA

Background: Huntington disease carries substantial psychiatric burden, including heightened risk for suicidal behaviors. Most suicidality literature in $\mathrm{HD}$ is based on natural history studies or retrospective reviews, but reports on risk factors from clinical trials are limited.

Methods: We analyzed data from 2CARE, a randomized, doubleblind, placebo-controlled clinical trial with up to 5 years of follow-up, for risk factors related to suicidality. The primary outcome variable was the time from randomization until the first occurrence of either suicidal ideation or attempt. We also considered time from randomization until the first suicide attempt as a secondary outcome variable.

Results: Depression, anxiety, bipolar disorder, antidepressant or anxiolytic use, and prior suicide attempt at baseline were associated with time to ideation or attempt. Baseline employment status, marital status, CAG repeat length, tetrabenazine use, and treatment assignment (coenzyme $\mathrm{Q}_{10}$ or placebo) were not associated with suicidality. Time-dependent variables from the UHDRS Behavioral Assessment were associated with time to suicidal ideation or attempt, driven mainly by items related to depressed mood, low self-esteem/guilt, anxiety, suicidal thoughts, irritability, and compulsions. Variables associated with time to suicide attempt alone were generally similar.

Conclusion: These data suggest psychiatric comorbidities in HD are predictive of suicidal behavior while participating in clinical trials, reinforcing the importance of clinical surveillance and treatment toward lessening risk during participation and perhaps beyond. Designing a composite algorithm for early prediction of suicide attempts in HD may be of value, particularly given anticipated trials aimed at disease modification are likely to be long-term. 
54

\section{Enroll-HD Platform Services}

\section{Contact:}

Tim McLean

Joint Leader Enroll-HD Platform

tim.mclean@enroll-hd.org

447738433165

7 Hillside Gardensn

Dolphinton

West Linton EH46 7AE

United Kingdom

The Enroll-HD Platform Team

CHDI

Enroll-HD is a global research platform. Key elements of the platform's infrastructure include global study management and governance, standardized informed consent forms (ICFs) and site contracts, uniform clinical site training, an integrated EDC and study database, and a userfriendly comprehensive web portal. This infrastructure supports the Enroll-HD study, a prospective, observational, longitudinal registry study of $\mathrm{HD}$, currently with $>18,000$ participants who have performed standardized clinical assessments and biosample collections from annual visits at over 165 clinical sites in 19 countries.

Resources and services of the platform that are made available to the HD research community include easily accessible periodic clinical datasets and associated biosamples. Consequent research output may be used to support future clinical studies, including protocol design.

A team of subject matter experts (SMEs) with experience in activities such as ICF development and translation, site agreements and financial payments, insurance, assessment scale licensing, IRB/ethics submissions, site staff training and certification via the platform training portal, database construction, and data monitoring is available to assist in the implementation of clinical studies.

Long-term working relationships with the participating clinical sites allow for well-informed clinical trial site selection and efficient feasibility as well as ongoing support for issue resolution and trial facilitation. The Enroll-HD platform's substantial registry of participants allows for powerful in silico screening to support participant recruitment.

A toolkit of platform resources, procedures, and template documents is in preparation. The provision of platform services, including the SME support, is managed by a team of Project Resource and Service Managers (PRSM Team). This team also facilitates the interface between clinical study and trial project teams or researchers and the Enroll-HD Platform.

\section{5}

\section{Identification and Characterization of circHTT, a Circular RNA from the HTT Locus}

\section{Contact:}

Alan Monziani

Master's Student

alan.monziani@studenti.unitn.it

+393387841327

Via Max Valier 7/

39055, San Giacomo di Laives, BZ

Italy

Correspondence to: marta.biagioli@unitn.it

A. Monziani ${ }^{1}$, Takshashila Tripathi ${ }^{1}$, Francesca di Leva ${ }^{1}$, E. Kerschbamer $^{1}$, V. Mattis ${ }^{2}$, C. Dieterich ${ }^{3}$, Erik Dassi ${ }^{4}$, Hana Hansíková $^{5}$, Ferdinando Squitieri ${ }^{6}$, M. Biagioli ${ }^{1}$
${ }^{1}$ NeuroEpigenetics Laboratory, Centre for Integrative Biology-CIBio, University of Trento, Trento, Italy; ${ }^{2}$ The Board of Governors Regenerative Medicine Institute, Cedars-Sinai Medical Center, Los Angeles, CA, USA; ${ }^{3}$ Section of Bioinformatics and Systems Cardiology, University Hospital Heidelberg, Heidelberg, Germany; ${ }^{4}$ Computational Biology Laboratory, Centre for Integrative Biology-CIBio, University of Trento, Trento, Italy; ${ }^{5}$ Laboratory for Mitochondrial Disorders, Department of Pediatrics, Charles University, Prague, Czech Republic; ${ }^{6}$ Casa Sollievo della Sofferenza Research Hospital in San Giovanni Rotondo and in CSSMendel Institute of Human Genetics, Rome, Italy

Huntington disease (HD) is a hereditary, fatal neurodegenerative disorder caused by a CAG trinucleotide repeat expansion within exon 1 of the HTT gene. Recent findings revealed that the process of alternative splicing (AS) might be compromised in HD. AS regulation is crucial not only to the establishment of a repertoire of protein coding isoforms extremely relevant for the proper physiological characteristics of the nervous system but also to the biogenesis of circular RNAs (circRNAs), unusually stable, highly expressed noncoding RNAs produced by the circularization of exons which seems to have important roles during neuronal development and functioning. In this study, we identified the first ever reported circular RNA originating from the HTT locus (circHTT, Ex 2-6, chr4:30886653109150). This circRNA is expressed in the whole body but predominantly in the CNS (whole brain, cerebellum, spinal cord). We, then, demonstrated that circHTT is expressed in iPS-derived neuronal cell lines (neural progenitors and cortical neurons) where, significantly, circHTT expression seems to be upregulated by CAG expansion. Since circHTT was detected in whole blood, peripheral blood mononuclear cells, and red blood cells of healthy and HD individuals, we are currently evaluating circHTT itself as potential HD biomarker in blood and lymphocytes. On the other hand, because RNA circles have been reported to regulate the transcription and/or translation of their linear counterparts, we are testing whether overexpression of circHTT in mouse striatal cell lines (Q7/7, Q7/ 111, Q111/111) will affect WT and mut $H t t$ expression or modify wellknown phenotypes (ATP/ADP ratio, sensibility to oxidative stress) described in this well-established HD experimental model. These observations will potentially identify a new tool - a circular RNA molecule - to modulate huntingtin expression and, eventually, some cellular phenotypes associated with the expression of the mutant allele, thus possibly paving the way to new trials of therapeutic intervention.

56

Educating Patients and Caregivers on the Neuropsychiatric Symptoms of HD

Contact:

Sarah Moroz

sarah.moroz@vumc.org

(978) $837-1211$

389 Monroe St.

Nashville, TN 37208

United States

Sarah Moroz, David Isaacs, Jessie Sellers, Daniel Claassen

Vanderbilt University Medical Center Movement Disorders Clinic Nashville, TN, USA (all authors)

Background: As many as $87-99 \%$ of patients with Huntington disease (HD) suffer from at least one neuropsychiatric symptom during the course of their disease. Such symptoms include depression, anxiety, irritability, apathy, impulsivity, perseveration, and psychosis. Despite the prevalence and negative impact on patient and caregiver quality of life, these neuropsychiatric symptoms remain under-recognized and undertreated. We 
postulate that lack of patient and caregiver education hampers identification and communication to providers.

Methods: We have developed several informational handouts on neuropsychiatric manifestations of $\mathrm{HD}$ and distributed them in our HD Center of Excellence Clinic. Three handouts in particular were selected for this study: one a general overview of neuropsychiatric symptoms and two others discussing, respectively, depression, and anxiety in HD. Handouts were distributed to patients and their caregivers in clinic, and participants were asked to review the information at home. Before receiving the handouts and 1 week following their clinic visit, we conducted a short interview to assess participants' knowledge on some of the symptoms discussed. During the follow-up interview, we also evaluated how useful and/or helpful participants found the handouts.

Results: Patients and caregiver scores improved on the follow-up knowledge assessments. Similarly, the handouts were well received and regarded as accessible and informative. Qualitative feedback was collected from participants and will be utilized to further optimize the handouts. Conclusion: Using the data collected, we hope to conduct a more comprehensive study in the future, involving more participants and more handouts. So far, the results have been promising.

\section{7}

Yeliva (ABC294640), an Inhibitor of Sphingosine Kinase 2, Protects Against Neurotoxicity in Huntington Disease Models

\section{Contact:}

Jose Felix Moruno-Manchon

Postdoctoral Fellow

jose.felix.morunomanchon@uth.tmc.edu

832-680-9806

6431 Fannin Street

Houston, TX 77030

United States

Jose F. Moruno-Manchon ${ }^{1}$, Erin E. Furr-Stimming ${ }^{2}$, Louise D. McCullough $^{2,3}$, Andrey S. Tsvetkov ${ }^{1,3,4}$

${ }^{1}$ Department of Neurobiology and Anatomy, the University of Texas McGovern Medical School, Houston, TX, USA; ${ }^{2}$ Department of Neurology, the University of Texas McGovern Medical School, Houston, TX, USA; ${ }^{3}$ The University of Texas Graduate School of Biomedical Sciences, Houston, TX, USA; ${ }^{4}$ UTHealth Consortium on Aging, the University of Texas McGovern Medical School, Houston, TX, USA

The polyQ expansion confers toxic functions to huntingtin (Htt), and identifying modifiers of mutant $\mathrm{Htt}$ ( $\mathrm{mHtt}$ )-mediated neurotoxicity has been proposed as a therapeutic strategy for Huntington disease (HD). Sphingosine kinases 1 (SK1) and 2 (SK2) synthesize sphingosine-1-phosphate (S1P), a bioactive lipid messenger critically involved in many vital cellular processes such as autophagy and transcription. In non-neuronal cells, the SK2/S1P complex functions as a transcriptional repressor. We found that SK2 is nuclear in primary neurons and overexpressed SK2 is neurotoxic in a dose-dependent manner. SK2 promotes DNA double-strand breaks in cultured primary neurons. We also found that SK2 is hyperphosphorylated in the brain samples from a model of $\mathrm{HD}$, the BACHD mice. These data suggest that the SK2 pathway may be a part of a pathogenic pathway in HD. An inhibitor of SK2 (Yeliva (ABC294640)) reduces DNA damage in neurons and increases survival in two neuron models of HD. We also discovered that SK2 coimmunoprecipitates with wild-type Htt. We hypothesize that the polyQ expansion in the Htt protein disrupts the SK2-Htt complex, freeing SK2. As a result, SK2, not bound to Htt, over-represses transcription, including transcription of DNA repairing genes. These questions are currently being pursued in our laboratory. SK2 might be a promising target for therapeutic development in HD and other disorders, in which DNA damage is present. Support: Hereditary Disease Foundation
58

Huntington Disease Regulatory Science Consortium (HD-RSC): Accelerating HD Therapies Through Collaboration, Regulatory Science, and Innovation

Contact:

Ariana Mullin

Assistant Director, Huntington's Disease Regulatory Science Consortium amullin@c-path.org

520-382-9045

1730 East River Road

Tucson, AZ 85718

United States

Ariana P. Mullin ${ }^{1}$, Diane Stephenson ${ }^{1}$, Jackson Burton ${ }^{1}$, Klaus Romero ${ }^{1}$, Emily Gantman ${ }^{2}$, Cristina Sampaio ${ }^{2}$

${ }^{1}$ Critical Path Institute, Tucson, AZ, USA; ${ }^{2}$ CHDI Management/CHDI Foundation, Princeton, NJ, USA

Objective: The Huntington Disease Regulatory Science Consortium (HD-RSC) was established to build regulatory pathways to accelerate the approval of HD therapeutics by advancing therapeutic area data standards and drug development tools.

Background: Recent scientific advancements and a greater understanding of HD pathophysiology have led to an emerging HD therapeutic landscape, yet drug developers still face high risks and many challenges remain. Critical Path Institute (C-Path) and CHDI Foundation have formed the HD-RSC to develop a regulatory science strategy and address the urgent needs in HD drug development.

Methods: Building on C-Path's successes in fostering global publicprivate partnerships, the HD-RSC will act as a neutral third party in bringing together representatives from industry, academia, regulatory agencies, and patient advocacy organizations to share data, learnings, and expertise. The HD-RSC will integrate patient-level data from HD observational studies and clinical trials to build an integrated database of standardized HD patient level data. Through interrogation of this database, the HD-RSC will develop and advance drug development toolsincluding models, biomarkers, and clinical outcome measures - for use in clinical trials.

Results: HD-RSC was launched on 28 March 2018 by C-Path in collaboration with CHDI Foundation with 13 founding industry members. In collaboration with the Clinical Data Interchange Standards Consortium (CDISC), the HD-RSC has developed the first consensus HD-specific data standards for clinical trial use. Use of clinical data standards by trial sponsors is now required by regulatory agencies to aid the regulatory review of new drug submissions. Concepts outlined in this HD therapeutic area user guide (HDTAUG) include biomarkers, genetics, and clinical measures.

Conclusions: Precompetitive data sharing and data integration within the HD-RSC will accelerate drug development by leading to the identification of appropriate clinical trial endpoints, design, and overall innovation in HD regulatory science.

\section{9}

\section{Real-World Experience with Novel VMAT2 Inhibitors}

\section{Contact:}

Nicki Niemann, MD

niemann@bcm.edu

713-798-8513

7200 Cambridge St Suite 9A

Houston, TX 77098

United States 
Nicki Niemann, Joseph Jankovic

Parkinson's Disease Center and Movement Disorders Clinic, Department of Neurology, Baylor College of Medicine, Houston, TX, USA (both authors)

Objective: This study aims to review real-world experience with novel vesicular monoamine transporter 2 (VMAT2) inhibitors, including tetrabenazine (Xenazine, and generic), deutetrabenazine (Austedo), and valbenazine (Ingrezza), in the treatment of various hyperkinetic movement disorders including chorea in Huntington disease.

Background: VMAT2 inhibitors have received FDA approval for the treatment of chorea in Huntington disease (tetrabenazine, 2008; deutetrabenazine, 2017) and tardive dyskinesia (deutetrabenazine, 2017; valbenazine, 2017) in recent years (Bashir and Jankovic, Expert Rev. Nerother. 18:51-63, 2018; Niemann and Jankovic, Drugs 78:525-541, 2018). Access to these therapies, however, is often limited by insurance denial, especially for "off-label use," high cost, lack of clinical efficacy, and emergence of adverse effects. Our short- and long-term experiences with tetrabenazine for the treatment of hyperkinetic movement disorders has been summarized in prior publications (Palao et al., Mov Disord. Suppl 2:S259, 2011; Kenney et al., Mov Disord. 22:193-197, 2007; Jankovic and Beach, Neurology 48:358-362, 1997; Kenney et al., Mov Disord. 22:10-13, 2007).

Methods: We developed a questionnaire to assess the patient's experience with any VMAT2 inhibitor prescribed for a movement disorder in our clinic between 30th August 2017 and 30th August 2018. The questionnaires will be submitted to the patients starting 30th August 2018, which marks 1 year since deutetrabenazine received FDA approval for tardive dyskinesia.

Results: Our prior report of tetrabenazine in 185 patients with chorea in Huntington's disease, revealed that tetrabenazine was well tolerated, efficacious, and accessible to the vast majority of patients ${ }^{3}$. Since the beginning of the study period, deutetrabenazine and valbenazine were prescribed to a total of 89 patients with an average age of 41.6 years (range, 4-85) and average initial target dose of 24.8 and $75.6 \mathrm{mg} /$ day, respectively. Of these, $51.7 \%$ received insurance approval, $36.0 \%$ were denied, and the status of $12.4 \%$ is pending or unknown. The most common indications were Tourette syndrome (46.1\%), tardive dyskinesia (29.2\%), and chorea in Huntington's disease (15.7\%).

Conclusion: Although patients seem pleased with the overall experience of VMAT2 inhibitors for various hyperkinetic movement disorders, many have not been able to access for "off-label" use.

1. Bashir H, Jankovic J. Treatment options for chorea. Expert Rev. Neurother. 2018;18:51-63.

2. Niemann N, Jankovic J. Treatment of tardive dyskinesia: A general overview with focus on the vesicular monoamine transporter 2 inhibitors. Drugs. 2018;78:525-541.

3. Palao A, Hunter C, Jankovic J. Patients' perspective of tetrabenazine

2 years after FDA approval. Mov Disord. Epub 2011.:Suppl 2:S259.

4. Kenney C, Hunter C, Jankovic J. Long-term tolerability of tetrabenazine in the treatment of hyperkinetic movement disorders. Mov Disord. 2007;22:193-197.

5. Jankovic J, Beach J. Long-term effects of tetrabenazine in hyperkinetic movement disorders. Neurology. 1997;48:358-362.

6. Kenney C, Hunter C, Davidson A, Jankovic J. Short-term effects of tetrabenazine on chorea associated with Huntington's disease. Mov Disord. 2007;22:10-13.

60

Individual Perception of Severity of Positive Genetic Test Results and the Use of Social Media

\section{Contact:}

Virginia Norris

Genetic Counselor/Research Coordinator

virginia.norris@vcuhealth.org
804-627-1398

PO Box 980539

Richmond, VA 23298-0539

United States

Kaitlyn E. Riley ${ }^{1}$, Virginia W. Norris ${ }^{2}$, Virginia Pallante ${ }^{1}$, John Quillin ${ }^{1}$, Kathleen S. Arnos ${ }^{3}$

${ }^{1}$ Department of Human and Molecular Genetics, Virginia Commonwealth University, Richmond, VA, USA; ${ }^{2}$ VCU Parkinson's and Movement Disorders Center, Virginia Commonwealth University, Richmond, VA, USA; ${ }^{3}$ Department of Biology, Gallaudet University, Washington, DC, USA

With a dramatic increase in the use of social media and genetic testing, more people are posting their genetic test results on social media. Social media is useful for connecting individuals who are geographically isolated, but there is a lack of research on motivations for posting online and the possible benefits or repercussions. In this study, individuals who have tested positive for the Huntington disease (HD) or BRCA1/2 mutations were surveyed to determine perceived severity of their test results, if they posted online, and motivations for posting or not-posting. One hundred five individuals (94\% female; average age range, 41-45) completed the survey, mostly recruited through the Huntington Disease Society of America, Huntington Disease Youth Organization, and Facing Our Risk of Cancer Empowered social media pages. Perceived severity was assessed using a modified scale based on the Health Belief Model (Cronbach alpha $=0.89$ ), with possible scores from 13 (low severity) to 65 (high severity). Perceived severity was higher for HD participants ( $p=$ 0.012 ). HD participants were less likely to have posted their genetic test results on social media (34 versus $73 \% ; p<0.001$ ). Perceived severity appeared to be inversely associated with posting, but this did not reach statistical significance $(p=0.112)$. Reasons individuals chose to post their results included to spread awareness of their condition, as well as to provide and receive support. Over $76 \%$ of people who posted reported benefitting, with most reporting they felt supported and less alone. Approximately $14 \%$ reported experiencing negative repercussions, specifically feeling a loss of privacy and being treated differently by others. Data from this study demonstrated that genetic counselors and other healthcare providers should be prepared to discuss the benefits and risks of posting genetic information online, as well providing information about online support groups in addition to in-person support groups.

\section{1}

\section{Huntington Disease and Amyotrophic Lateral Sclerosis: a Case Study}

\section{Contact:}

Gillian Owens

Genetic Counselor

gillian.blaber@nygh.on.ca

416-756-6787

4001 Leslie Street

3SE

Toronto ON M2K1E1

Canada

Gillian Owens, Sherali Esmail, Harrison Mah, Wai Lun Alan Fung, Clare Gibbons

North York General Hospital, Toronto, Ontario, Canada-University of Toronto, Toronto, Ontario, Canada (all authors)

Background: Huntington disease (HD) and amyotrophic lateral sclerosis (ALS) are clinically distinct neurodegenerative disorders; however, there 
are reports of the coexistence of the two conditions. This has led to a debate about whether these cases are a coincidental occurrence of both diseases or whether an expanded HD allele could be a risk factor for developing ALS in a rare subset of HD patients.

Case History: We report a male patient with onset of HD motor symptoms at 64 years of age who then developed ALS at 74 years of age. The patient has multiple family members with HD including his father who developed symptoms in his early $70 \mathrm{~s}$. The patient had predictive testing for HD in 1998, which revealed an expanded HTT allele of 39 CAG repeats. He has no family history of ALS. The patient reported HD symptoms began about 8 years ago and recently some mild cognitive decline. A motor exam in 2014 found mild chorea in all extremities, head, and neck. Swallowing difficulties and worsening chorea and balance were noted in 2016. By November 2017, the patient presented with rapidly progressing proximal weakness in the arms and legs, as well as, distal weakness and atrophy in the right hand intrinsics and weakness at both ankle dorsiflexors. The degree of weakness and wasting was higher than typically seen in HD. Further examination found fasciculation in the distal hands. Needle EMG examination showed mild denervation in the right C5/C6 myotome and severe denervation in the right tibialis anterior. In addition, there was evidence of wide spread denervation in the thoracic paraspinal muscles in keeping with active and chronic motor neuron disease.

Conclusion: We present a patient with early manifest HD who developed ALS to contribute to the literature in this area.

\section{2}

\section{Natural Language Processing (NLP) of Verbatim Consequence Patient-Reported Outcomes Highlight Functional Ability as Important to Patients}

\section{Contact:}

Jennifer Purks

Medical Student

jlp92@georgetown.edu

781-275-9838

3911 Benton Street NW

Washington, DC 20007

United States

Jennifer L. Purks ${ }^{1}$, Michael Harris ${ }^{1}$, Karen E. Anderson ${ }^{1}$, Ira Shoulson ${ }^{1,2}$

${ }^{1}$ Georgetown University, Washington, DC, USA; ${ }^{2}$ University of Rochester, Rochester, NY, USA Funding Support; Prana Biotechnology Ltd., Melbourne, Australia

Financial Disclosures: Ira Shoulson serves as nonexecutive director of Prana Biotechnology Ltd. Karen Anderson is a paid consultant to Prana Biotechnology Ltd.

Objective: This study aims to apply the Natural Language Processing (NLP) tools of word clouds (visual single-word count frequency depiction) and tri-grams (frequency of three words reported sequentially together) to analyze verbatim consequence of patient-reported outcomes (PROs) among Huntington disease (HD) research participants in the phase 2 REACH2HD randomized-controlled trial examining safety and cognitive benefits of PBT2.

Background: FDA and other regulatory agencies value PROs, but analytic approaches are lacking. The Huntington Disease Patient-Reported Outcome of Problems (HD-PROP) was developed to capture verbatim reports of patients' most bothersome problems and functional consequences due to HD. The HD-PROP was administered at baseline (BL) and 26 weeks (W26) of the REACH2HD trial to the 109 research participants (randomly assigned 1:1:1 to PBT2 $250 \mathrm{mg}$ /day, PBT2 $100 \mathrm{mg}$ /day, or placebo).
Methods: Using NLP to extract relationships and meaning, word clouds and tri-grams of verbatim consequences associated with verbatim bothersome problems were analyzed at BL and W26 for each treatment group. Results: Across all time and treatment groups, consequences word clouds displayed "things" 111 counts, "get" 68 counts, and "like" 42 counts. The most frequently reported tri-grams at baseline for all three groups reported decline from prior ability, such as "it takes me longer" and "I used to." At week 26, all three groups continued to report decline in prior function. Conclusion: NLP can quantify functional patterns in the REACH2HD trial verbatim HD-PROP dataset. The tri-gram is clinically more meaningful compared with a single word count because it provides context for consequences reported. Most commonly reported tri-grams demonstrated that PROs in this dataset prioritize both ability and temporal changes in functioning and offer examples of how clinical trial patients verbalize accommodation, decline, or improvement in functioning associated with HD. More advanced NLP methods could further refine analysis of PROs in HD and other movement disorders.

\section{3}

Legato-HD Study: a Phase 2 Study Assessing the Efficacy and Safety of Laquinimod as a Treatment for Huntington Disease

Contact:

Pippa Loupe

Director, Academic Affairs and Networks

pippa.loupe@tevapharm.com

785-218-7049

Teva Pharmaceuticals

2205 Riviera Drive

Lawrence, KS 66047

United States

\section{Presenter:}

Ralf Reilmann

Founding Director George-Huntington-Institute

ralf.reilmann@ghi-muenster.de

+49-251-788-788-0 (coordinator)

George-Huntington-Institute Technology-Park Muenster

Johann-Krane Weg 27

Muenster 48149

Germany

Ralf Reilmann ${ }^{1}$, Mark Forrest Gordon ${ }^{2}$, Karen E. Anderson ${ }^{3}$, Andrew Feigin $^{4}$, Sarah J. Tabrizi ${ }^{5}$, Blair R. Leavitt ${ }^{6}$, Julie C. Stout ${ }^{7}$, Paola Piccini $^{8}$, Gail Rynkowski ${ }^{2}$, Rita Volkinshtein ${ }^{2}$, Juha Savola ${ }^{2}$

${ }^{1}$ George-Huntington-Institute Muenster and Department of Neurodegenerative Diseases and Hertie Institute for Clinical Brain Research University of Tübingen, Tübingen, Germany; ${ }^{2}$ Research and Development, Teva Pharmaceuticals Ltd.; ${ }^{3}$ MedStar Georgetown University Hospital and Georgetown University Medical Center, Washington, DC, USA; ${ }^{4} \mathrm{NYU}$ Langone Health New York, NY; ${ }^{5}$ UCL Institute of Neurology London; ${ }^{6}$ Centre for Molecular Medicine and Therapeutics University of British Columbia, Vancouver, BC, Canada; ${ }^{7}$ Monash University, Melbourne, Australia; ${ }^{8}$ Imperial College London, London, UK

Objective: This study aims to evaluate the efficacy and safety of laquinimod in patients with HD.

Methods: The LEGATO-HD study originally included three dose arms, $0.5 \mathrm{mg}, 1.0 \mathrm{mg}$, and $1.5 \mathrm{mg}$ versus placebo, in a 12 -month multicenter double-blind phase 2 study in patients with HD. Cardiovascular safety concerns were observed in multiple sclerosis studies with laquinimod doses of 1.2 and $1.5 \mathrm{mg}$. Although no similar concern was identified in LEGATO-HD, Teva discontinued the $1.5 \mathrm{mg}$ arm in January 2016 as a 
precautionary safety measure and continued to evaluate the efficacy and safety of the 0.5 and $1.0 \mathrm{mg}$ doses. Efficacy assessments include the primary endpoint, change from baseline in Unified Huntington's Disease Rating Scale Total Motor Score (UHDRS-TMS) at month 12, and the secondary endpoint, percent change in caudate volume at month 12. Exploratory endpoints include changes in MRI volume measures and changes in Q-Motor, CIBIC-Plus, UHDRS-Total Functional Capacity (TFC) and UHDRS-Functional Assessment (FA) scores. Safety measures include adverse event reporting, clinical laboratory tests, vital signs, ECGs, physical examinations, and suicidality (C-SSRS).

Results: Three hundred fifty-two patients were randomized. The last patient visit occurred in June 2018. Baseline mean \pm S.D. pooled demographics of enrolled patients include females $n=172 \pm 49.1 \%$, males $n=178 \pm 50.9 \%$, age $44.4 \pm 7.6$ years, UHDRS-TMS $24.3 \pm 13.1$, UHDRS-TFC $11.1 \pm 1.7$, and UHDRS-FA $22.7 \pm 2.4$. Results of primary, secondary, and exploratory efficacy endpoints, along with safety findings, will be presented at the conference.

Conclusion: There is a significant unmet medical need to ameliorate symptoms and the progressive neurodegeneration in HD. LEGATO-HD provides valuable information toward understanding the efficacy and safety of laquinimod as a potential treatment for patients with HD.

Funding: The LEGATO-HD Study is sponsored by Teva Pharmaceuticals Ltd. in collaboration with HSG and EHDN and is registered as NCT02215616-clinicaltrials.gov and 2014-000418-75-EudraCT.

\section{4}

\section{Blood: a Transmission Route of Mutant Huntingtin Protein}

\section{Contact:}

Marie Rieux, MSc

$\mathrm{PhD}$ Student

marie.rieux.1@ulaval.ca

+1418-271-3979

714 rue d'Aiguillon

Appartement 2

Quebec QC G1R 1M7

Canada

Marie Rieux, Martine Saint-Pierre, Melanie Alpaugh, Abid Oueslati, Steve Lacroix, Francesca Cicchetti

Centre de Recherche du CHU de Québec-Université Laval, Québec, Québec, Canada (all authors)

In recent years, we have published data indicating that mutant huntingtin (mHtt) is capable of spreading within the nervous system, inducing a Huntington-like behavior phenotype in mice lacking the genetic huntingtin mutation. A recent study using parabiosis, which connected transgenic mice for Alzheimer's disease to wild-type (WT) mice, demonstrated that blood-derived $A \beta$ protein can cross the blood-brain barrier and spread within the brain of WT mice; data suggesting the implications of blood in the transport of pathological proteins.

We investigated whether $\mathrm{mHtt}$ can also spread via the blood to peripheral organs or from the periphery to the central nervous system, and if this can further seed pathology and accelerate disease phenotype in the context of Huntington disease.

To do this, we used the parabiosis model to join zQ175 HD mice to wildtype (WT) mice. Blood content and peripheral organs were analyzed at several time points $(3,6,9$, and 12 months after surgery) by immunoblotting and immunohistochemistry. We observed the presence of $\mathrm{mHtt}$ in various blood components (platelets, erythrocytes, and leucocytes) as early as 5 days after surgery and during the entire protocol, i.e., up to 12 months following the parabiotic surgery. Significant quantities of free forms of soluble $\mathrm{mHtt}$ were also detected in the plasma of WT parabionts. To date, immunohistochemical analyses suggest that as early as 3 months after surgery, $\mathrm{mHtt}$ aggregates are present within the muscle, liver, and kidney of WT mice paired to ZQ175.

The use of parabiosis will allow us to confirm the contribution of the circulatory system to $\mathrm{mHtt}$ dissemination, as demonstrated by our preliminary data. This technique will further allow us to determine if rejuvenating blood factors from the healthy WT parabiont can alleviate HD-related pathology/phenotype in transgenic animals.

\section{5}

\section{Decreased Cerebral Blood Flow in the Striatum Is an Early Event in Huntington Disease Pathology}

\section{Contact:}

Natalia P. Rocha, PhD

natalia.pessoarocha@uth.tmc.edu

713-486-2622

1941 East Road

Suite 3270

Houston, TX 77054

United States

Natalia P. Rocha ${ }^{1,2}$, Leigh B. Latham ${ }^{2,3}$, Odelin Charron ${ }^{3}$, George Papadimitropoulos $^{3}$, Gabriela D. Colpo ${ }^{1}$, Leorah Freeman ${ }^{3}$, Erin Furr Stimming $^{2,3}$, Antonio L. Teixeira ${ }^{1}$

${ }^{1}$ Department of Psychiatry and Behavioral Sciences, McGovern Medical School, The University of Texas Health Science Center at Houston (UTHealth), Houston, TX, USA

${ }^{2}$ HDSA Center of Excellence at University of Texas Health Science Center at Houston, Houston, TX, USA; ${ }^{3}$ Department of Neurology, McGovern Medical School, The University of Texas Health Science Center at Houston, Houston, TX, USA

Rationale: The ultimate cause of neuronal death in Huntington disease (HD) is still uncertain. Apart from impairment in systems for handling abnormal proteins, other mechanisms, such as inflammation, might contribute to neurodegeneration and progression of HD. Cerebral hypoperfusion has been described in neurodegenerative disorders such as Alzheimer's disease, and in traumatic brain injury, and may play a role in HD.

Objective: This study was designed to investigate cerebral blood flow $(\mathrm{CBF})$ changes in HD patients and premanifest HD gene carriers.

Methods: Eighteen patients with genetic diagnosis of HD (11 manifest patients $(48.6 \pm 10.9$ years; $3 \mathrm{M} / 8 \mathrm{~F}), 7$ premanifest HD gene carriers $(47.3 \pm 9.4$ years; $2 \mathrm{M} / 5 \mathrm{~F}))$, and 13 controls $(32.4 \pm 2.4$ years; $7 \mathrm{M} / 6 \mathrm{~F})$ underwent brain magnetic resonance imaging (MRI) at $3 \mathrm{~T}$ with pseudo-continuous arterial spin labeling (pCASL) to quantify $\mathrm{CBF}$, and 3D-MPRAGE for volumetric segmentation. pCASL images were analyzed with FMRIB Software Library (FSL) and FreeSurfer v5.3.0. Region of interest (ROI) analyses were performed to compare $\mathrm{CBF}$ in controls versus premanifest HD gene carriers versus manifest patients with HD (Kruskal-Wallis test followed by Dunn's multiple comparisons test).

Results: Both premanifest HD gene carriers and manifest patients had a significant decrease in CBF in deep gray matter (DGM) in comparison with controls $(p=0.037)$. There was a significant decrease in CBF in bilateral caudate and putamen of manifest patients with HD in comparison with controls. The same changes were observed in premanifest HD gene carriers with a mean of 8 years before the predicted onset of clinical symptoms ( $p=0.035$ and $p=0.003$ for left and right caudate, respectively; and $p=0.029$ and $p=0.012$ for left and right putamen, respectively). Conclusions: Our preliminary results suggest that hypoperfusion may be an early event in HD and precedes motor symptom onset. Decreased CBF 
and oxygen supply could emerge as a link between neuroinflammation and degeneration in HD.

Funding: NPR is a Huntington's Disease Society of America (HDSA) fellowship recipient.

67

Neuroprotective Effects of Zaprinast Against Quinolinic AcidEvoked Neurotoxicity in Experimental Model of Huntington Disease

\section{Contact:}

Priyanka Saroj

Research Scholar

priyanka1960@gmail.com

+91-9456989656

Girls Hostel No-3

Panjab University, Chandigarh

Chandigarh ID 160014

India

Priyanka Saroj, Yashika Bansal, Raghunath Singh, Sangeeta P. Sah, Anurag Kuhad

Pharmacology Lab No-13, University Institute of Pharmaceutical Sciences Panjab University, Chandigarh 160014, India (all authors)

Huntington disease (HD) is an autosomal, fatal, progressive neurodegenerative disorder that causes clinical manifestations such as progressive choreiformic movements, cognitive abnormality, and psychiatric deterioration. In HD, aggregates of mutant huntingtin (mhtt) found in neurons, astrocytes, and microglia result in increased production of proinflammatory cytokines viz. IL-1 $\beta$, IL-2, IL-6, IL-8, and tumor necrosis factor (TNF- ) but decreased production of anti-inflammatory cytokines viz., IL-3, IL-4, IL-5, and IL-10. In HD patients, an impaired cyclic nucleotide signaling and its downstream cAMP response element-binding protein (CREB) transcriptional pathways have also been reported. Phosphodiesterase- 5 has been implicated in various neurological diseases like $\mathrm{HD}, \mathrm{PD}$, and $\mathrm{AD}$ as a secondary messenger to cAMP signaling. In our study, administration of quinilonic acid (QA) $(200 \mathrm{nmol} / 2 \mu \mathrm{l}$ saline) caused neurobehavioral (locomotor, motor coordination, and muscle grip strength) and biochemical alterations that mimic Huntington disease in humans. Treatment with zaprinast significantly ameliorated the QA-induced oxidative/nitrosative stress and neuroinflammation in brain striatum and cortex. Treatment of zaprinast reversed the mitochondrial dysfunction and neurochemical/ neurotrophic (BDNF) changes in brain. In conclusion, these results revealed the neurotherapeutic potential of zaprinast in Huntington disease.

68

Phenotype of Patients with Intermediate Length CAG Repeats in the Huntingtin Gene

Contact:

Daniel Savitt, DO

daniel.savitt@bcm.edu

713-798-2273

7200 Cambridge

9th Floor

Houston, TX 77030

United States

Daniel Savitt, Joseph Jankovic

Baylor College of Medicine Houston, TX, USA (all authors)
Objective: This study aims to describe the phenotype of patients with intermediate length CAG repeats (IA) in the huntingtin (HTT) gene seen at the Parkinson's Disease Center and Movement Disorders Clinic (PDCMDC) at Baylor College of Medicine (BCM).

Background: Huntington disease (HD) is caused by a mutation in the HTT gene leading to 40 or more CAG trinucleotide repeats. Since our original case report of pathologically proven HD with 29 CAG repeats (Kenney et al., Mov. Disord. 22(1):127-130, 2007), a growing body of evidence has accumulated supporting the observation that individuals with IA may exhibit clinical, imaging, and pathologic manifestations of HD. About $6 \%$ of the general population have IAs in the 27 to 35 range in at least one allele in the HTT gene, and the HD new mutation rate is highest in populations with the highest frequency of IAs (Kay et al., Am J Med Genet B Neuropsychiatr Genet 177(3):346-357, 2018). The presence of IA presents a challenge for genetic counseling.

Designs/Methods: Medical records of patients with IAs seen at the PDCMDC at BCM from January 2008 to the present were reviewed to assess age at symptom onset, dominant clinical features, and presence of psychiatric and cognitive symptoms.

Results: Five male and four female individuals were evaluated and found to have IAs (range, 27-32). The age at onset of clinically significant symptoms ranged from 27 to 78 years. Six patients had chorea, three had gait disturbances, two had stereotypies, and one patient had multiple motor tics. All nine patients had psychiatric symptoms, with depression being the most common and two patients had functional (psychogenic) movement disorders.

Conclusion: Our series of patients with IA in the HTT gene exhibit a variety of motor and nonmotor features that overlap with the HD phenotype. These individuals and their offspring should be considered at risk for development of progressive HD.

1. Kenney C, Powell S, Jankovic J. Autopsy-proven Huntington's disease with 29 trinucleotide repeats. Mov. Disord. 2007;22(1):127-130.

2. Kay C, Collins JA, Wright GEB, et al. The molecular epidemiology of Huntington disease is related to intermediate allele frequency and haplotype in the general population. Am J Med Genet B Neuropsychiatr Genet. 2018;177(3):346-357.

\section{9}

\section{Mortality in the Cooperative Huntington Observational Research Trial (COHORT)}

\section{Contact:}

Ruth Schneider

Assistant Professor of Neurology

ruth_schneider@urmc.rochester.edu

585-273-1856

265 Crittenden Blvd.

Box MIND

Rochester, NY 14642

United States

\section{R.B. Schneider, P. Auinger}

University of Rochester, Rochester, NY, USA (all authors)

Background: Huntington disease (HD) is associated with a reduced life expectancy. Heart disease has been consistently identified as a leading cause of death in HD, and both autonomic nervous system dysfunction and cardiac electrical remodeling have been identified in HD. However, the incidence of heart disease-related death is not greater than that of the general population.

Methods: This retrospective analysis examined medical events, number of deaths, causes of deaths, and time to death (Kaplan-Meier) in HD in the Cooperative Huntington Observational Research Trial (COHORT), a 
multicenter, prospective observational study of 1514 individuals with clinically manifest HD.

Results: Of the clinically manifest HD participants, $52.6 \%$ were female and $93.0 \%$ were Caucasian. The mean \pm S.D. age was $50.8 \pm 12.4$, time since clinical diagnosis was $4.2 \pm 4.1$ years, CAG length was $43.7 \pm 5.5$, Unified Huntington's Disease Rating Scale total motor score was $36.1 \pm$ 19.5, and Total Functional Capacity score was $8.5 \pm 3.4$. The mean duration of follow-up was $2.7 \pm 1.4$ years. Over the course of the study, 61 deaths $(4.0 \%)$ occurred and the mean \pm S.D. age of death was $58.1 \pm$ 16.9 years. The rate of death at 1 year was 9.5 per 1000 individuals. The most commonly identified cause of death was pneumonia (18\%). Cardiac arrest or thromboembolism accounted for $8 \%$ of deaths. Eighty-three $(5.5 \%)$ individuals reported a history of coronary heart disease, cerebrovascular disease, peripheral artery disease, venous thromboembolism, or thrombophilia.

Discussion: In COHORT, the overall incidence of cardiovascular and cerebrovascular disease was low among individuals with HD and accounted for only a small percentage of the deaths that occurred. In comparison, in the USA in $2015,23.4 \%$ of deaths were attributed to cardiovascular disease and $5.2 \%$ to cerebrovascular disease. More research is needed to explore the presence and significance of cardiac dysfunction in HD.

Funding Information: This research was supported by Nuredis, Inc.

\section{0}

\section{The Autonomic Nervous System as a Potential Therapeutic Target in Huntington Disease}

\section{Contact:}

Jordan Schultz, PharmD

Clinical Pharmacy Specialist

jordan-schultz@uiowa.edu

309-798-9084

200 Hawkins Drive

$\mathrm{CC} 101 \mathrm{GH}$

Iowa City, IA 52242

United States

Jordan L. Schultz, John A. Kamholz, Annie Killoran, Alexander Tereshchenko, Joel Bruss, Allison Bernard, Timothy A. Koscik, and Peg C. Nopoulos

University of Iowa, Iowa City, IA, USA (all authors)

Objective: This study (1) investigated the presence and severity of autonomic nervous system (ANS) dysfunction in patients with Huntington disease (HD) and (2) determined if pharmacologic manipulation of the ANS could modify the progression of HD.

Methods: Using a unique data set of children at-risk for HD (the KidsHD study), markers of autonomic function (resting heart rate (rHR), blood pressure, and core body temperature) were compared between presymptomatic, gene-expanded children (psGE), and healthy developing children using mixed-model analyses controlling for sex and age. Using the Enroll-HD database, propensity score-adjusted, Cox regression analyses investigated the effects of ANS-modulating medications on the timing of motor diagnosis of presymptomatic, adult patients with HD.

Results: Compared with healthy controls, the psGE participants had significantly $(p<0.05)$ higher rHR and core body temperatures (elevated by $3.23 \mathrm{bpm}$ and $0.15^{\circ} \mathrm{C}$, respectively, in the psGE group). Participants from Enroll-HD who were using sympathomimetic medications (serotonin/ norepinephrine reuptake inhibitors or stimulants) had a significantly $(p<0.05)$ higher annualized risk of motor diagnosis compared with participants not using these medications. Furthermore, participants who were using a beta-blocker prior to motor diagnosis $(n=78)$ demonstrated a significantly lower annualized risk of motor diagnosis $(\mathrm{HR}=0.57$ (95\% CI, 0.38-0.96)l $p=0.031$ ), compared with other participants with HD $(n=2289)$.

Discussion: Sympathetic nervous system activity is elevated in patients with HD, and sympathomimetic medications seem to hasten motor onset of HD. Furthermore, modulation of the sympathetic nervous system with $\beta$-blockers significantly lowers the annualized risk of motor diagnosis of HD.

71

Comparing Risperidone and Olanzapine to Tetrabenazine for the Management of Chorea in Huntington Disease

\section{Contact:}

Jordan Schultz, PharmD

Clinical Pharmacy Specialist

jordan-schultz@uiowa.edu

309-798-9084

200 Hawkins Drive

$\mathrm{CC} 101 \mathrm{GH}$

Iowa City, IA 52242

United States

Jordan L. Schultz, John A. Kamholz, Peg C. Nopoulos, Annie Killoran

The University of Iowa, Iowa City, IA, USA (all authors)

Introduction: Little evidence supports the use of neuroleptic medications for Huntington's chorea (HC). This study aimed to perform a real-world comparison of the efficacy of risperidone and olanzapine to tetrabenazine (TBZ) for HC

Methods: The Enroll-HD database was used to perform a propensity score-matched comparison of risperidone and olanzapine to TBZ, in regard to their efficacy in controlling chorea. Participants with motor manifest Huntington disease (HD) were grouped according to their use of risperidone, olanzapine, or TBZ. Participants who were using TBZ were first matched with risperidone users and subsequently with olanzapine users with two independent propensity score matches. A third propensity score match compared the risperidone and olanzapine users. Participants were matched based on their baseline total functional score, baseline total motor score, disease burden score, CAG repeat length, baseline age, region, sex, and body mass index. After each match, the differences in the annual rate of change of the total motor score (TMS) between the baseline visit and second available visit between the groups was calculated using independent samples $t$ tests.

Results: The risperidone group $(n=72)$ had an annualized increase (worsening) in TMS of 1.47 points compared with 5.70 points in the matched TBZ group $(n=72 ; p=0.019)$. The olanzapine group $(n=77)$ had an annualized increase in TMS of 3.20 compared with 5.70 points in the matched TBZ group $(n=77 ; p=0.143)$.

Conclusions: These results demonstrate that neuroleptics have comparable efficacy with TBZ for the treatment of chorea, but these results must be confirmed in a randomized controlled trial.

\section{2}

Tetrabenazine and Acceleration of Huntington Disease

\section{Contact:}

Jordan Schultz, PharmD

Clinical Pharmacy Specialist

jordan-schultz@uiowa.edu

309-798-9084 
200 Hawkins Drive

CC101 GH

Iowa City, IA 52242

United States

Jordan L. Schultz, John A. Kamholz, David Moser, Annie Killoran, Matthew McIlrath, Jonathan Doorn, and Peg C. Nopoulos

University of Iowa, Iowa City, IA, USA (all authors)

Objective: Tetrabenazine (TBZ) is a vesicular monoamine transport-2 (VMAT-2) inhibitor used to manage chorea in Huntington disease (HD). It increases intracellular dopamine and increases the production of toxic metabolites within dopaminergic neurons. This study investigated if TBZ use was associated with hastened clinical progression in HD. Methods: This was a retrospective analysis of the Enroll-HD database. Participants were included if they had a CAG repeat length of at least 36, at least two documented Enroll-HD visits, and a diagnostic confidence level of four at their baseline Enroll-HD visit. Participants were divided according to their TBZ use. Propensity score matching was employed to decrease heterogeneity between groups. Comparisons were made between the TBZ users and the TBZ nonusers in regard to the annual rates of change in the total motor score (TMS), the total function capacity (TFC), the symbol digit modalities test (SDMT), and the Stroop Word Reading test (SWRT) scores.

Results: The TBZ users had faster annual rates of decline for all outcome measures compared with the TBZ nonusers. The difference in the rates of decline between groups was 4.26 points per year (ppy) for the TMS ( $p=$ $0.001), 0.58$ ppy for the TFC ( $p=0.008), 4.11$ ppy for the SWRT ( $p=$ $0.007)$, and 1.64 ppy for the SDMT $(p=0.009)$.

Discussion: Participants with HD who were taking TBZ demonstrated significantly faster rates of clinical progression of HD compared with matched participants who were not taking TBZ. We hypothesize that this accelerated decline is secondary to enhanced production of toxic dopamine metabolites within the dopaminergic neurons.

73

\section{Machine Learning Approach in Analysis of Enroll-HD Data for} Suicidality Prediction in Huntington Disease

Contact:

Yury Seliverstov

doctor.goody@gmail.com

$+79167992228$

Volokolamskoe shosse, 80

Moscow

Russian Federation 125367

Yury Seliverstov $^{1}$, Artyom Borzov ${ }^{2}$, Erik van Duijn ${ }^{3}$, Bernhard Landwehrmeyer $^{4}$, Mikhail Belyaev ${ }^{5}$

${ }^{1}$ Research Center of Neurology, Moscow, Russia; ${ }^{2}$ Institute for Information Transmission Problems, Moscow, Russia; ${ }^{3}$ Leiden University Medical Centre, Leiden, Netherlands; ${ }^{4}$ Ulm University Hospital, Ulm, Germany; ${ }^{5}$ Skolkovo Institute of Science and Technology, Moscow, Russia

Background: Suicidal ideation and suicidal behavior are frequently reported, severe features in Huntington disease gene expansion carriers (HDGECs), but it is difficult to predict who are at increased risk.

Objective: This study aims to develop a model for prediction of suicidal ideation/behavior in HDGEC based on Enroll-HD data using machinelearning approach (MLA).

Design/Methods: We have developed a prediction model based on MLA using the third Enroll-HD study periodic dataset (PDS3). Suicidal ideation/behavior was measured with the Columbia-Suicide Severity Rating Scale (C-SSRS). HDGECs with no or "passive" suicidal ideations (state 1) at their first visit, who at the follow-up (FUP) either stayed in state 1 or worsened to active suicidal ideations and/or suicidal behavior (state 2) were analyzed. The PBAs scale was used to assess presence of behavioral symptoms. Prediction algorithm was based on Boosted Trees (implementation from XGBoost Library for Python) and contained 48 variables from the PDS3.

Results: For 377 HDGECs (114 premanifest; 161 males; median age, 50 $(20 ; 78)$; median $\mathrm{nCAG}=43(38 ; 65))$, C-SSRS data of two consecutive visits were available. At the FUP, 316 remained in state 1 and 61 HDGECs had worsened to state 2. Sixty-four percent of the HDGECs who remained in state 1 at FUP were accurately classified (probability as having state $2<30 \%$ ). HDGECs who worsened to state 2 were correctly predicted in $38 \%$ cases (probability of being classified as having state $2>$ $60 \%$ ). We then compared the poorly (probability $<30 \% ; 31$ subjects) with the well (probability $>60 \% ; 23$ subjects) classified groups in state 2 at FUP and found significant differences in the PBA total scores for depression, anxiety, aggression, and apathy (more severe baseline scores in the well-classified HDGECs).

Conclusions: Our model showed moderate accuracy. Further research is needed to understand risk of suicidal ideation/behavior in HDGECs with mild behavioral symptoms.

\section{4}

\section{Neuropsychiatric Symptoms and Functional Capacity in Huntington's Disease}

\section{Contact:}

Jessie Sellers

Assistant in Neurology

jessie.sellers@vumc.org

615-343-4744

116121 st Avenue S.

A-0118 Medical Center North

Nashville, TN 37232

United States

J. Sellers, S. Ridner, D. Claassen

Vanderbilt University, Nashville, TN, USA

Objective: This study aims to review existing literature on the relationship of neuropsychiatric symptoms with functional capacity in HD.

Background: Neuropsychiatric symptoms are known to increase caregiver burden and decrease patient quality of life in HD. Functional capacity is an outcome commonly used in HD clinical trials to quantify disease progression or intervention response. Some studies have examined the relationship between neuropsychiatric symptoms and functional capacity, but this evidence has not been synthesized. We sought to review existing evidence on the association between neuropsychiatric symptoms and functional capacity in HD.

Methods: We completed a systematic review on Medline using search terms "Huntington's disease" AND ((psychiatric OR neuropsychiatric OR behavioral OR behavioral) AND symptom*) AND (function OR (functional AND (capacity OR status OR ability OR performance))). Articles were included if they described primary research in humans with $\mathrm{HD}$, measured one or more neuropsychiatric symptoms and functional capacity, and reported statistical methods to look for associations between the two concepts. Articles were excluded if TFC was used only to classify disease stage and not specifically as a measure of functional capacity. Additional eligible articles were identified through reference mining and review of other relevant literature. 
Results: We identified 14 eligible articles. Neuropsychiatric symptoms were measured individually, in clusters (i.e., depression, anxiety, and suicide items contributing to a depression cluster score) or with an overall score. Significant associations with decreased functional capacity were found with depression $(n=7$; median $r=0.545)$ and apathy $(n=3$, median $r=0.21$ ) most commonly. Other neuropsychiatric symptoms, clusters, and overall scores were each associated with functional capacity in two or fewer studies.

Conclusions: There is some evidence that depression and apathy are associated with decreased functional capacity in HD. Other neuropsychiatric symptoms have been infrequently examined. Further knowledge of the relationships between neuropsychiatric symptoms and functional capacity will identify areas for intervention and improvement of outcomes in HD patients.

75

Atypical Antipsychotics for Neuropsychiatric Symptoms and choREa in HD (ANSWER-HD): Protocol and Preliminary Data

\section{Contact:}

Jessie Sellers

Assistant in Neurology

jessie.sellers@vumc.org

615-343-4744

116121 st Avenue S

A-0118 Medical Center North

Nashville, TN 37232

United States

\section{J. Sellers, D.O. Claassen}

Vanderbilt University Medical Center, Nashville, TN, USA (all authors)

Objective: This study aims to present a protocol and preliminary data for a phase-III, multicenter, double-blind randomized controlled $2 \times 2$ crossover trial evaluating atypical antipsychotics for neuropsychiatric symptoms in Huntington disease (HD)

Background: Neuropsychiatric symptoms are common in HD, contributing to decreased patient quality of life and increased caregiver burden. Evidence for treatment of these symptoms in HD is poor and limited to expert opinion and noncontrolled trials. Antipsychotics (APs) are used by HD providers worldwide for neuropsychiatric symptoms and chorea, despite lack of strong evidence. Risperidone, olanzapine, and aripiprazole, particularly, are reported as useful for neuropsychiatric and motor symptoms in case studies and open-label trials, with fewer adverse effects than typical APs and sedating atypicals like quetiapine and clozapine. We propose to evaluate the efficacy of these agents for neuropsychiatric symptoms in a randomized, placebo-controlled trial. We will also include chorea reduction as an exploratory aim.

Methods: Our crossover trial will involve two arms: HD patients taking VMAT-2 inhibitors and those not taking VMAT-2 inhibitors. Participants in each arm will be randomized to begin the trial with an 8-week period of limited physician-choice AP (risperidone, olanzapine, or aripiprazole) or placebo. After a 2-week washout, participants will cross over to the other arm (placebo or AP). Site physicians will consider patient comorbidities and known medication side-effects when choosing each participant's AP. Participants and assessors will be blinded to 1) study condition in each 8week period and 2) which AP was chosen for the subject's AP period. Primary endpoint will be reduction of total Problem Behaviors Assessment shot form (PBA-s) score, whereas reduction in Total Maximal Chorea Score (TMC) will be a secondary, exploratory endpoint. Conclusion: We will present detailed information on study design and preliminary data gathered, using HSG seed funds, to support our hypothesis and guide sample size estimations.

\section{6}

In Vitro Validation of Antibody-Based Therapy to Neutralize Mutant Huntingtin Protein

Contact:

Alberto Siddu

PhD student

asiddu90@gmail.com

+1418997 7475

Rue Samuel-King 941 apt 903

Quebec City

QC G1V 0G1

Canada

Siddu Alberto ${ }^{1,3}$, David Linda Suzanne ${ }^{1,3}$, Smzka Oskar $^{4}$, Bartl Stefan ${ }^{4}$, Queslati Abid ${ }^{2,3}$, Cicchetti Francesca ${ }^{1,3}$

${ }^{1}$ Département de Psychiatrie \& Neurosciences, Faculté de Médecine, Université Laval, Quebec City, QC, Canada; ${ }^{2}$ Département de Médecine Moléculaire, Université Laval, Quebec City, QC, Canada; ${ }^{3}$ Centre de Recherche du CHU de Québec-Université Laval, CHUL, Quebec City, QC, Canada; ${ }^{4}$ AFFiRiS AG, Vienna, Austria

We believe that three important facts have been largely ignored when it comes to the development of novel therapies for Huntington disease (HD): (1) mutant huntingtin protein $(\mathrm{mHtt})$ is often present extracellularly, notably in the cerebrospinal fluid, plasma, and the matrix, (2) $\mathrm{mHtt}$ is expressed outside the CNS leading to the manifestation of clinical symptoms associated to peripheral organs, and (3) mHtt can spread between cells in a prion-like fashion. These observations open the door for the development of antibody-based therapies that can target free/extracellular $\mathrm{mHtt}$ and potentially arrest the spread of pathogenic $\mathrm{mHtt}$. To test this possibility, we designed an in vitro paradigm in which an experimental antibody targeting the $\mathrm{N}$-terminal of $\mathrm{Htt} / \mathrm{mHtt}$ (C6-17; AFFiRiS) was introduced to bind and neutralize the extracellular mHtt present in conditioned media, thereby stopping its uptake by healthy cells in culture. We overexpressed constructs of the N-terminal of Htt or mHtt in Hek293T (donor cells); the conditioned media containing these proteins was harvested and then exposed to HeLa (acceptor cells) in the presence of C6-17 or control antibodies (IgG2a) to evaluate the ability of C6-17 to block uptake of $\mathrm{mHtt}$. We evaluated the outcome of our study in five individual sets of replicates using Western blot; we not only observed the presence of various forms of $\mathrm{Htt} / \mathrm{mHtt}$ in the conditioned media, but that C6-17 is able to bind/neutralize and block the uptake of $\mathrm{mHtt}$ from the milieu by healthy acceptor cells. These results were further confirmed by the quantification of intracellular $\mathrm{mHtt}$ aggregates after immunofluorescence in HeLa cells. Our results demonstrate that antibodies can be employed to stop the spread of $\mathrm{mHtt}$ between cells, supporting the development of immunotherapy.

\section{7}

Handwriting Movement Abnormalities as an Early Biomarker of Transition to Manifest Huntington's Disease

Jody Corey-Bloom

Professor of Neurosciences

jcoreybloom@ucsd.edu

858-249-0574

Department of Neurosciences

9500 Gilman Drive MC0948

La Jolla, CA 92093-0948

United States 
Chase Snell ${ }^{1}$, Jody Corey-Bloom ${ }^{1}$, Sungmee Park ${ }^{1}$, Michael Caligiuri ${ }^{2}$ ${ }^{1}$ Neurosciences, UC San Diego, La Jolla, CA, 92093 USA; ${ }^{2}$ Psychiatry, UC San Diego, La Jolla, CA, 92093 USA

Objective: The objective of the current study was to examine whether handwriting movement abnormalities are present prior to clinically manifest chorea in Huntington disease (HD) and may serve as an early biomarker of transition to manifest disease.

Background: Prior research has demonstrated that measures of handwriting kinematics are sensitive to mild preclinical motor abnormalities stemming from other basal ganglia disorders. The presence of a biomarker early in the transition period in HD may help efforts to pharmacologically manage or delay onset.

Design/Methods: Twenty-five gene-positive premanifest (PM), 35 symptomatic HD, and 15 healthy control (HC) subjects completed handwriting tasks consisting of circles, loops, sentences, and spirals with a non-inking pen on a standard digitizing tablet. Multiple measures of pen stroke kinematics and pressure were extracted from these trials. The clinical status of each participant was characterized using the UHDRS and several disease burden scores, including Burden of Pathology (BOP) and CAG/age product (CAP) scores.

Results: HD subjects exhibited significantly longer and more variable stroke durations, decreased smoothness, and increased more variable pen pressures compared with the $\mathrm{HC}$ subjects over multiple handwriting tasks. Among the more robust correlations between handwriting kinematics and clinical variables, we found significant positive associations between BOP and stroke duration, CAP and stroke duration, and UHDRS Total Motor Score and pen pressure variability. With regard to predictive modeling, results from a discriminant function analysis revealed an 8factor model that distinguished PM from $\mathrm{HC}$ subjects with $87 \%$ accuracy $(F=8.13 ; p<0.0001)$. Factors in the model included greater variability in stroke amplitude, velocity, and pen pressure, higher levels of pen pressure, longer stroke durations, and lower velocities for combinations of handwritten circles, sentences, and spirals.

Conclusions: These findings support the clinical utility of dynamic measures of handwriting kinematics as a potential early biomarker in HD.

77

\section{Mutant Huntingtin Preferentially Induces DNA Damages in the Actively Transcribing Genes by Inactivating DNA Strand Break Repair Enzyme PNKP}

\section{Contact:}

Jeffrey Snowden

jesnowde@utmb.edu

972-533-4240

117 Tarpon Ave.

Galveston, TX 77550

United States

Jeffrey Snowden ${ }^{1}$, Rui Gao ${ }^{1}$, Anirban Chakraborty ${ }^{2}$, Kara L. Gordon ${ }^{3}$, Tetsuo Ashizawa ${ }^{4}$, Albert R. La Spada ${ }^{3}$, Tapas K. Hazra ${ }^{2}$, Partha S. Sarkar ${ }^{1}$

\footnotetext{
${ }^{1}$ Department of Neurology and Neuroscience and Cell Biology; ${ }^{2}$ Department of Internal Medicine, University of Texas Medical Branch, Galveston, TX, USA; ${ }^{3}$ Departments of Neurology, Duke University School of Medicine, Durham, NC, USA; ${ }^{4}$ Department of Neurology, Houston Methodist Research Institute, Houston, TX, USA
}

The pathological mutation in Huntington disease (HD) is the expansion of a polyQ tract at the N-terminus of huntingtin due to the expansion of a CAG triplet repeat in the mutant huntingtin ( $m H T T$ ) gene, which leads to progressive deterioration of cognitive and motor functions. However, the mechanism by which expanded polyQ sequences in mHTT cause neuronal dysfunction and neurodegeneration remains unknown. We report that wild-type (WT) HTT forms a multiprotein transcription-coupled DNA repair (TCR) complex with DNA strand break repair enzyme polynucleotide kinase (PNKP), deubiquitinase ataxin 3 (ATXN3), RNA polymerase II subunit A (POLR2A), cyclic AMP response element-binding (CREB) protein (CBP), and several transcription factors. This novel TCR complex identifies lesions in the template DNA strand and mediates their repair during transcriptional elongation. By contrast, the polyQ expansion in mHTT abrogates PNKP and ATXN3 activities, disrupting the functional integrity of the TCR complex, adversely impacting both TCR and transcription. Impaired PNKP activity leads to reduced DNA repair and accumulation of DNA lesions, predominantly in transcriptionally active regions, resulting in aberrant activation of ATM-dependent pro-apoptotic p53 signaling. Increasing PNKP activity in the mutant cells substantially decreased DNA lesions, blocked aberrant ATM $\rightarrow$ p53 pathway activation, and dramatically improved cell survival. Moreover, our findings demonstrate that mHTT-mediated abrogation of ATXN3 activity increases ubiquitination and degradation of CBP, negatively influencing CREB-dependent transcription. Collectively, these findings provide new insights into how mHTT-mediated abrogation of PNKP and ATXN3 activities impairs TCR, simultaneously disrupting transcription, inducing DNA strand breaks, and activating the pro-apoptotic DDR$\mathrm{ATM} \rightarrow \mathrm{p} 53$ signaling pathway to trigger neurotoxicity. Our results also suggest potential targets for developing therapeutic modalities to combat neurodegeneration and neuronal dysfunction in HD.

\section{9}

\section{A Novel Objective Measure for Measuring Chorea in Huntington Disease}

Contact:

Christopher Snyder

Clinical Research Coordinator

chris.snyder@chet.rochester.edu

585-815-3749

University of Rochester Center for Health and Technology

265 Crittenden Blvd CU 420694

Rochester, NY 14692

United States

Karthik Dinesh ${ }^{3}$, Christopher Snyder ${ }^{2}$, Mulin Xiong ${ }^{2}$, Christopher G. Tarolli $^{1,2}$, Saloni Sharma ${ }^{2}$, Ray Dorsey ${ }^{1,2}$, Gaurav Sharma ${ }^{3,4}$, Jamie L. Adams ${ }^{1,2}$

${ }^{1}$ Department of Neurology, University of Rochester Medical Center, Rochester, NY, USA; ${ }^{2}$ Center for Health + Technology, University of Rochester Medical Center, Rochester, NY, USA; ${ }^{3}$ Department of Electrical and Computer Engineering, University of Rochester Medical Center, Rochester, NY, USA; ${ }^{4}$ Department of Computer Science, University of Rochester, Rochester, NY, USA

Current measures for quantifying Huntington's chorea such as the Unified Huntington Disease Rating Scale (UHDRS) are subjective and categorical and generally limited to episodic in-person visits. These limited-duration, subjective assessments might fail to capture actual chorea severity, which would complicate the clinical management of chorea and evaluation of drug efficacy. Few studies have employed objective measures to quantify the motor symptoms of Huntington disease. We report on a novel objective measure for chorea using a wearable sensor. Wearable sensors, which can enable objective, frequent, remote, and longitudinal data collection, can enhance monitoring of chorea and other symptoms.

Using the accelerometer data obtained from sensors worn in clinic and at home, we developed a biomarker called the "Chorea Index," which quantifies truncal chorea in individuals with Huntington disease. Fourteen individuals with Huntington disease and nineteen controls were included 
in the analysis. We collected sensor data using over the course of a 1-h inclinic visit and a 2-day at-home monitoring period. With the Chorea Index, we can track the variation of chorea both in clinic and at home. The index differentiates between voluntary motion and involuntary motion, and the score represents the total amount of involuntary motion. At this stage, the index provides the most reliable measurement of total chorea while the individual is seated. The chorea value generated by this algorithm correlates well with the trunk chorea score returned from the clinician-rated UHDRS maximal truncal chorea score $(r=0.86 ; p<0.01)$. Although the in-clinic and at-home chorea assessments show a similar trend, we were able to detect intrascore chorea symptom variability among those with Huntington disease between the two settings. This Chorea Index could be instrumental in determining drug efficacy and tracking the progression of Huntington disease over time.

80

Huntington's Disease in the Arabic Peninsula: a Report from the Sultanate of Oman

\section{Contact:}

Ferdinando Squitieri

ferdinandosquitieri@yahoo.it

+393396425285

CSS-Mendel Institute (IRCCS Casa Sollievo della Sofferenza Research Hospital)-Viale Regina Margherita, 261

00198 Rome

Italy

Ferdinando Squitieri ${ }^{1,3}$, Hamda Al-Hajri ${ }^{2}$, Mohammed Al-Hajri ${ }^{2}$, Barbara D'Alessio ${ }^{3}$

${ }^{1}$ IRCCS Casa Sollievo della Sofferenza Research Hospital (Rome CSSMendel), San Giovanni Rotondo, Italy; ${ }^{2}$ Oman HD Association, Muscat, Sultanate of Oman; ${ }^{3}$ Lega Italiana Ricerca Huntington e malattie correlate (LIRH) Foundation, Rome, Italy

Background: Some geographical areas are poorly studied and would need more conceivable clinical genetic analyses. One example is the Arabic Peninsula, where HD cases have only been sporadically described.

Methods: Since August 2013, LIRH Foundation has been working to raise awareness of HD among patients, physicians, and institutions in response to a request from several members of a large HD kindred with six generations from Oman. Because Arabic people are used to marrying within the same family, the mutation risk is reiterating in offspring. A Neurogenetic Centre with outpatient and molecular diagnostic services focused on HD was launched by the Ministry of Health at Royal Hospital, Oman, in February 2014. The Oman HD Association of families was also set up to increase awareness and improve clinical assistance for patients. Findings: In this six-generation family, we visited 23 symptomatic subjects of 42 reported by genealogical retrospective analysis and several $50 \%(n=84), 25 \%(n=86)$ and $>50 \%(n=18)$ at-risk individuals due to the many intermarriages. DNA testing was provided, after informed consent, with the support of genetic counseling, and clinical data were collected by the Unified HD Rating Scale. Age at onset ranged between 2 and 79 years (CAG) and between 39 (oldest onset) and 174 (earliest onset) repeats. Such families showed a mean age of onset that is younger than European ancestries of Italian origin, faster disease progression, and higher JHD frequency (i.e., 14/23, 17\%). A profound feeling of isolation among the many young at-risk people in this area was clearly registered. Conclusion: This is a cross-sectional and longitudinal analysis of a middle-eastern HD population. Our analysis will contribute to understanding additional, yet unsolved, clinical (i.e., JHD), genetic (i.e., where the HD mutation is originating from), and social (i.e., how Arab population is dealing with HD) traits of HD.
81

Effect of Adipose-Derived Mesenchymal Stem Cell on Neuroinflammation in the Preymptomatic Stage of Huntington Disease

\section{Contact:}

Amit K. Srivastava

Assistant Professor

amit.k.srivastava@uth.tmc.edu

713-500-7253

6431 Fannin Street

Rm 5.252

Houston, TX 77030

United States

Amit K. Srivastava ${ }^{1}$, Naama E. Toledano Furman ${ }^{1}$, Akshita Kumar ${ }^{1}$, Alex Villarreal $^{1}$, Karthik S. Prabhakara ${ }^{1}$, Molly Leitner ${ }^{1}$, Gabriela D. Colpo ${ }^{2}$, Andrey S. Tsvetkov ${ }^{3}$, Antonio L. Teixeira Jr. ${ }^{2}$, Erin Furr-Stimming ${ }^{4}$, Scott D. Olson ${ }^{1}$

${ }^{1}$ Department of Pediatric Surgery, University of Texas Health Science Center at Houston, McGovern Medical School, Houston, TX, USA; ${ }^{2}$ Department of Psychiatry \& Behavioral Sciences The University of Texas, Houston Medical School, Houston, TX, USA; ${ }^{3}$ Department of Neurobiology and Anatomy, The University of Texas, Houston Medical School, Houston, TX, USA; ${ }^{4}$ Department of Neurology, The University of Texas Health Science Center at Houston, Houston, TX, USA

Huntington disease (HD) is a fatal, neurodegenerative disease characterized by progressive motor dysfunction, cognitive decline, and psychiatric symptoms. Developing relevant therapeutic interventions for $\mathrm{HD}$ has proven to be challenging, particularly due to the insidious, neurodegenerative course of the disease. Pathophysiology of HD is complex and although HD is characterized by a predominant loss of neurons in the striatum, previous studies point to a critical role of neuroinflammation in disease progression. Several studies have presented strong evidence that microglia activation, a hallmark of neuroinflammation, is detected in the brains of presymptomatic HD carriers to postmortem HD patients. The microglial activation in HD also correlates with disease severity and the loss of vast majority of striatal neurons. In this study, we examined the therapeutic effect of a commercial preparation of adipose-derived mesenchymal stem cells (HB-AdMSCs) on neuroinflammation in transgenic HD mice in the presymptomatic stage. The cells were obtained from Hope Biosciences (Sugar Land, TX) and intravenously infused either in B6CBA-R6/2 transgenic HD mice or wild-type mice. No immunosuppressive drug was given to any animal. The animals were euthanized 7 days after the cell injection to harvest the brains, and microglia were analyzed using multiparametric flow cytometry to profile their accumulation, activation, and polarization. We observed that HB-AdMSCs were able to reduce neuroinflammation by reducing microglial activation in HD mice compared with controls. The results of this study may be used to develop HB-AdMSC-based therapy for neuroinflammation associated with other neurological disorders as well.

\section{2}

\section{HD Clinical Trial Site Certification-the Enroll-HD Platform}

\section{Contact:}

Jenny Townhill, MD

Jenny.Townhill@enroll-hd.org

$+447791985313$

47 Newton Nottage Road.

Porthcawl CF36 5RR

United Kingdom

Enroll-HD Platform

CHDI Foundation 
The certification scheme aims to maximize the number of high-quality, trial-ready sites; improve efficiency; and expedite the site selection process for clinical trials and studies. It provides an opportunity for sites to register their interest and ability to participate in trials and increases the visibility of potential trial sites for sponsors and clinical study teams. The scheme reduces the administrative burden for sites and sponsors during trial set-up by centrally storing information about site feasibility, training, qualifications, and experience and provides these details to approved sponsors. Guidance and support is provided for less-experienced sites to become trial ready.

Certification requires that sites meet a specific set of criteria designed to assess clinical trial readiness, including general site information, clinical trial infrastructure, patient cohort, training, and education. The scheme is open to Enroll-HD and non-Enroll-HD sites in Australia, Europe, Latin America, North America, New Zealand, Nordic regions, and Russia. The core information for the certification application is collected using the HD Global Site Investigator Database (GSID-HD) and requires the submission of a standard set of documents and SOPs for review. For further information, contact hdsite@euro-hd.net.

83

Validity, Reliability, and Ability to Detect Change and Meaningful Within-Patient Change of the cUHDRS

\section{Contact:}

Matt Gooding

Matt.Gooding@meditechmedia.com

+442073980557

Admiral House

76-78 Old Street

London EC1V 9AZ

United Kingdom

\section{Presenter:}

Dylan Trundell, MD

dylan.trundell@roche.com

+442073980557

Roche Products Ltd.

Welwyn Garden City AL7 1TW

United Kingdom

Dylan Trundell ${ }^{1}$, Giuseppe Palermo ${ }^{2}$, Scott $_{\text {Schobel }}{ }^{3}$, Jeffery D. Long ${ }^{4,5}$, Blair R. Leavitt ${ }^{6}$, Sarah J. Tabrizi ${ }^{7}$

${ }^{1}$ Roche Products Ltd., Welwyn Garden City, UK; ${ }^{2}$ Roche Pharma Research and Early Development, Roche Innovation Center Basel, Basel, Switzerland; ${ }^{3}$ F. Hoffman-La Roche Ltd., Basel, Switzerland; ${ }^{4}$ Department of Psychiatry, Carver College of Medicine, University of Iowa, Iowa City, IA, USA; ${ }^{5}$ Department of Biostatistics, College of Public Health, University of Iowa, Iowa City, IA, USA; ${ }^{6}$ Centre for Molecular Medicine and Therapeutics, Department of Medical Genetics, University of British Columbia, Vancouver, BC, Canada; ${ }^{7}$ Huntington's Disease Research Centre, UCL Institute of Neurology, London, UK

Background: Composite Unified Huntington's Disease Ratings Scale (cUHDRS) is a combined score of measures of motor function (TMS), cognition (SDMT and SWR), and overall functional capacity (TFC). cUHDRS was developed to assess multidomain clinical progression in Huntington disease (HD) and was shown to be sensitive, reliable, and valid. To support its use in clinical studies, further evidence is required. This includes estimates of clinically meaningful change of the cUHDRS and its individual measures.

Aims: This study aims to assess the reliability, validity, and ability to detect change of the cUHDRS and that of its individual measures and to estimate minimal clinically meaningful within-patient cUHDRS change.

Methods: Data from an early manifest HD population (TFC $\geq 5$ ) from two multinational registries (ENROLL-HD and REGISTRY) were used. Test-retest reliability was assessed by calculating the intraclass correlation coefficient in a subset of patients with no change in Clinical Global Impression of Severity (CGI-S) score. Convergent validity was assessed by Spearman rank-order correlations. Known-group validity was assessed by analysis of covariance (ANCOVA) between groups defined by CGI-S. Ability to detect change was assessed by ANCOVA comparing groups based on CGI-S score change. Regression analyses were conducted to estimate meaningful change, using CGI-S and Independence Scale (IS) as anchors. Finally, an alternative scoring algorithm of the cUHDRS based upon progressor definitions of its individual component measures was derived, and the performance of cUHDRS confirmed.

Results: Strong evidence of test-retest reliability, known-group validity, and ability to detect change were demonstrated. Convergent validity was supported by stronger correlations with measures that are more similar. Meaningful within-patient change was estimated.

Conclusions: cUHDRS is valid, reliable, and able to detect change in patients with HD stages 1-2. Analyses anchored against CGI-S and IS and progressor definitions support that a decline on cUHDRS is clinically meaningful.

Funding Information: This study was funded by F. Hoffmann-La Roche.

Lay Summary: HD has a range of symptom domains that affect day-today life, including movement, cognitive function, behavior, and functional capacity. Existing assessment scales used in HD clinical studies typically measure one symptom domain. The cUHDRS scoring system combines existing measurement scales to holistically measure patient experience. Using registry data, this study found that using cUHDRS in clinical studies is a reliable way to measure meaningful changes in disease progression and the potential effects of investigational drugs.

84

The Use of a Single-Focus Musical External Cue to Improve Functional Mobility in Individuals with Huntington Disease: a Pilot Study

Contact:

Elizabeth Ulanowski

Assistant Professor

eulanowski@bellarmine.edu

502-523-3145

4107 Saratoga Woods Drive

Louisville, KY 40299

United States

Elizabeth Ulanowski, Megan Danzl, Alyssa Brothers, Alexandra James, Lisa Schwartz, and Kaitlyn Starr

Bellarmine University, Louisville, KY, USA (all authors)

Purpose/Hypothesis: To date, there is no research exploring the best type of external cue for individuals with HD to improve movement patterns and safety. The purpose of this research report is to examine mobility in individuals with HD with and without a single-focus musical external cue provided through a drum by a music therapist (MT). It was hypothesized in this pilot study that the external cue would improve mobility.

Number of Subjects: Ten subjects living in a long-term care facility were initially recruited. Eight were ambulatory and able to participate in outcome measure assessments. The mean age was 49 years (range, 36-65), and all but one subject demonstrated chorea.

Materials/Methods: Subjects were evaluated by a physical therapist (PT) using the Timed Get Up and Go (TUG) and the 10-Meter Walk Test 
(10MWT) outcome measures. The MT observed the assessments to gain further insight into subject needs. The within-session data collection included outcome measures performed under two separate conditions: PT alone, followed by PT with MT and a drum. All outcomes were video recorded to complete a visual observational analysis of each subject's movement patterns. Results: Functional movement patterns notably improved in terms of movement control and diminished chorea with the use of the drumming cue in comparison with the PT-only administered test. Seven of eight participants increased their 10MWT times, and five of eight increased their TUG time when adding the single-focus external drumming cues.

Conclusions: The 10MWT and TUG results all indicated an increase in time with the drumming cue compared with without, which could suggest a decline in movement and safety. As subjects slowed and improved movement control with the drum cue, their test times increased. HD is the unusual case in which PTs may not aim to improve speed. These preliminary findings support consideration by a PT to integrate a single-focus external cue.

\section{5}

The Role of Music on Movement in Huntington Disease: a Physical and Music Therapy Partnership

\section{Contact:}

Elizabeth Ulanowski

Assistant Professor

eulanowski@bellarmine.edu

502-523-3145

4107 Saratoga Woods Drive

Louisville, KY 40299

United States

Elizabeth Ulanowski, Megan Danzl, Alyssa Brothers, Alexandra James, Lisa Schwartz, and Kaitlyn Starr

\section{Bellarmine University, Louisville KY, USA (all authors)}

Purpose: The implementation of music therapy in healthcare improves patient mood, movement patterns, and overall quality of life. To date, there is no research exploring the use of music therapy as a cotreatment with physical therapy in individuals with Huntington disease (HD). The purpose of this report is to describe a novel physical and music therapy approach to working with individuals with late-stage HD and to explore the role of music on functional movements.

Description: Ten participants with HD living in a long-term care facility were evaluated by a physical therapist (PT) including observation of basic functional movements. From this data, a selection of movements was chosen to trial with music. The treatment session included three functional movement performance conditions: physical therapy alone, physical and music therapy with a drum, and then a guitar. Observations of both sessions were collected in-person and through video analysis along with observational movement analyses from video review. After intervention, an interview was conducted with the PT and MT about their perceptions of the experience, which were then coded for meaning.

Conclusions/Summary of Use: Preliminary findings suggest the MT/PT cotreatment was beneficial using a simple auditory cue such as a drum from the MT during functional tasks directed by the PT. The drum allowed the MT to quickly evolve the rhythm to match the needs and variability of the pattern based on the chorea and movements. The cues provided allowed participants to demonstrate improved control over the hyperkinetic movements. The drum appears to be more effective than the guitar with participants demonstrating improved attention and responsiveness to the drum cue but additional research is warranted. Further study is needed to determine the effects and differences between uses of the auditory musical cue as a background cue versus a cognitively mediated approach.
86

Abnormal Brain Development in PreHD Children and Adolescents

\section{Contact:}

Peggy Nopoulos

Professor

peggy-nopoulos@uiowa.edu

319-384-9264

W278 GH

200 Hawkinds Drive

Iowa City, IA 52242

\section{Presenter:}

Ellen van der Plas

Professor

Ellen van der Plas, Vincent Magnotta, Sasha Tereshchenko, Jeff Long, Jim Mills, Peg Nopoulos

University of Iowa, Iowa City, IA, USA (all authors)

Huntington disease (HD) is considered a neurodegenerative disease, yet recent evidence suggests that neurodevelopmental factors play a role in disease. HTT is crucial for brain development. Developmentally compromised neurons (from $m H T T$ ) could maintain a mutant steady state throughout childhood and adolescence, before giving way to neurodegeneration.

The KidsHD program is a unique study of children at risk for HD. Participants are 6-25 years of age and either have a parent with HD, or are from the local community with no family history of HD. All children are genotyped and divided into those who are gene expanded (GE) or gene nonexpanded (GNE). GE children without symptoms represent those who are, on average, 30 years from onset of disease.

Brain MRI scans are obtained on $72 \mathrm{GE}$ children and compared with 235 GNE to examine if: (1) brain morphology is different between groups and (2) if the size of the cytosine-adenine-guanine (CAG) repeat expansion

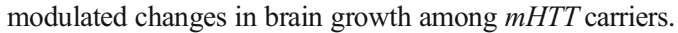

Analysis focused on the global measures of intracranial volume (ICV) and the nodes within the striatal-cerebellar circuit (caudate, putamen, globus pallidus, thalamus, cerebellum). Linear mixed effects regression (LMER) was used to groups and developmental trajectories. There were no group effects for ICV or the cerebellum. For the caudate, putamen, globus pallidus, and thalamus, there were significant group differences in growth trajectory based on CAG repeat length; those with the GE had steeper slopes of change between age 6 and 25, and in the GE group, the higher the CAG repeat, the steeper the slope.

mHTT affects development of the striatum and other subcortical structures in a CAG-dependent fashion with maturational processes having an accelerated change in the GE group compared with the control group, underscoring the role of abnormal development in HD.

\section{7}

\section{Model-Based Methods for Quantifying and Predicting Huntington's Disease Progression}

\section{Contact:}

Charles Venuto, Pharm.D

Assistant Professor

charles.venuto@chet.rochester.edu

585-276-5622

265 Crittenden Blvd., CU 420694, Suite 2406

Rochester, NY 14642-0694

United States 
Charles S. Venuto ${ }^{1,2}$, Shiyang $\mathrm{Ma}^{3}$, Robert L. Strawderman ${ }^{3}$, Karl Kieburtz $^{1,2}$, E. Ray Dorsey ${ }^{1,2}$, Christopher Snyder ${ }^{1}$, Monica Javidnia ${ }^{1,2}$, Peggy Auinger ${ }^{1,2}$

${ }^{1}$ Center for Health and Technology, University of Rochester, Rochester, NY, USA; ${ }^{2}$ Department of Neurology, University of Rochester, Rochester, NY, USA; ${ }^{3}$ Department of Biostatistics and Computational Biology, University of Rochester, Rochester, NY, USA

Objective: This study aims to understand the long-term natural history of early disease progression in Huntington disease (HD) and to reliably predict future disease severity scores as measured by either the Total Functional Capacity (TFC) scale or the composite Unified Huntington's Disease Rating Scale (cUHDRS) scores.

Methods: Participant-level data from three clinical trials (CARE-HD, 2CARE, and CREST-E) and one observational study (COHORT) were used to develop mixed-effects models for predicting longitudinal TFC and cUHDRS scores. A "classified mixed model prediction" was used for predicting future TFC and cUHDRS scores for an individual based on his or her baseline data by matching the individual to a particular "class" of similar HD individuals from the training dataset. Thus, the predictions are based on both fixed effects and random group effect.

Results: The dataset comprises 9536 TFC scores and cUHDRS scores each, from 2440 participants with early HD (TFC $>7$ ) for up to 60 months of follow-up. Baseline Total Motor Score, Body Mass Index, disease burden score (calculated as $(\mathrm{CAG}-35.5) \times$ age), and time since HD diagnosis were statistically significant covariates in the models for predicting both future TFC and cUHDRS scores $(p<0.05)$. Baseline age and CAG length of the participant were not statistically significant covariates. The accuracy and predictive capabilities of the models continue to be evaluated.

Conclusions: The proposed models are able to estimate future TFC and cUHDRS scores in early manifest HD patients using commonly collected clinical data. The utility of these models will be tested in clinical trial simulations that take advantage of being able to identify HD patients with slow and rapid disease progression trajectories.

\section{8}

\section{Pelvic Organ Function in Huntington Disease}

Contact:

Brenda Vicars

Research Registered Nurse

bvicars@uw.edu

206-598-8753

1959 NE Pacific St.

Boxes 356510

Seattle, WA 98195

United States

Brenda Vicars, Alice Liu, Suman Jayadev, Thomas Bird, Claire Yang

University of Washington School of Medicine Seattle, WA, USA (all authors)

Background: Previous understanding of HD identifies little association between HD and the development of lower urinary, bowel, and/or sexual dysfunction. Although complex and incompletely characterized, the neuroanatomy and innervation of the bladder, bowel, and genitalia are interrelated and normal functioning is dependent upon an intact central autonomic regulation, which is compromised in $\mathrm{HD}$.

In our University of Washington COE clinical experience, we have noted consistent patient reports of frequency, urgency, incontinence (both urinary and fecal), constipation, and sexual dysfunction among patients with
HD; however, association between symptoms and HD has not been closely evaluated. We report on the prevalence of pelvic organ symptoms in our cohort of patients with HD.

Methods: Fifty-six clinically symptomatic patients with HD were enrolled during a regularly scheduled clinic visit. All subjects were asked to complete questionnaires regarding bladder, bowel, and sexual function. Our cohort comprised 33 males, 23 females, age range 25-77 (average, 51), CAG range 40-61 (average, 44), TFC range 0-4 (average, 2.6), 29 married, 22 single, and 5 cohabitating. Fifty-six subjects completed the bladder and bowel questionnaires; 48 subjects completed the sexual function questionnaire.

Results:

\begin{tabular}{ll}
\hline Bladder function symptoms $n=56$ & $n(\%)$ \\
Frequency & $40(71)$ \\
Nocturia $(\geq 2 \times)$ & $27(48)$ \\
Urgency & $32(57)$ \\
Leakage & $31(55)$ \\
Bowel function symptoms $n=56$ & $n(\%)$ \\
A bowel problems & $32(57)$ \\
Constipation & $20(36)$ \\
Diarrhea & $16(29)$ \\
Bowel leakage & $12(21)$ \\
Sexual function $n=48$ & $n(\%)$ \\
Interest in sex & $40(83)$ \\
Has sex partner & $33(69)$ \\
Sexually active in last 6 months & $23(48)$ \\
Importance of sexual activity-very important & $14(21)$
\end{tabular}

Conclusions: There is a significant prevalence of pelvic organ dysfunction in patients with HD. Many pelvic organ symptoms are treatable and doing so can greatly enhance patients' quality of life.

89

Feasibility of Objectively Evaluating Huntington Disease's Motor Effects Via Smartphone Application

Contact:

Emma Waddell

Clinical Research Coordinator

emma.waddell@chet.rochester.edu

585-276-6825

265 Crittenden Blvd CU 420694

Rochester, NY 14642

United States

\section{Presenter:}

Alistair Glidden

Clinical Research Coordinator

alistair.glidden@chet.rochester.edu

585-275-2039

265 Crittenden Blvd CU 420694

Rochester, NY 14642

United States

Kelsey L. Spear, Christopher G. Tarolli, Emma M. Waddell, Molly J. Elson, Alistair M. Glidden, Tyler S. Geery, Michael J. Curtis, David J. Mitten, E. Ray Dorsey, Jamie Adams

Unless otherwise specified, all authors are: University of Rochester, Rochester, NY, USA; Molly J. Elson, Emory University, Atlanta, GA, USA 
Objective: This study aims to assess the feasibility of using an Android smartphone application (app), GEORGE®, to quantitatively measure factors of Huntington disease (i.e., gait, chorea, voice, balance, dexterity, bodily motion, mobility, and socialization).

Background: As technology becomes increasingly inexpensive, ubiquitous, and advanced, smartphones present a unique opportunity to measure disease. Previous studies of applications have demonstrated that smartphones effectively measure key characteristics of Parkinson disease, sensitively recognize daily variability and response to medication, and strongly correlate with the Unified Parkinson Disease Rating Scale.

Methods: GEORGE® has the potential to quantify motor symptoms, distinguish between individuals with and without $\mathrm{HD}$, and assess the response to drug treatment through more frequent monitoring. GEORGE® uses the smartphone's sensors to assess chorea and balance, the screen to assess tapping speed, the microphone to assess voice, and the GPS to assess socialization. Through faster data collection, this technology could expedite research studies and the development of novel therapeutics for HD and advance exploratory clinical trials to assess HD. Remote data collection will also ease the time and travel burden for research participants and caregivers.

Preliminary Results: This observational study of 40 participants, 20-25 with clinically diagnosed $\mathrm{HD}, 5-10$ with prodromal $\mathrm{HD}$, and 10 controls without a movement disorder, includes two in-clinic assessments and daily tasks for 1 month. To date, 14 subjects have been enrolled, 10 of whom have completed the study. Participants are 57\% women, $25 \%$ college educated, $42 \%$ employed, and $33 \%$ married. Fifty percent of participants have manifest HD. Preliminary data analysis is ongoing, and early results suggest that the accuracy of the chorea activity could be improved, but that use of the smartphone application is feasible for those with HD.

Future Directions: We will further refine the activities and user interface of GEORGE® based on participant feedback and data and lay the foundation for its use in evaluating the efficacy of current and future therapies for HD

90

Subtle Verbal Memory Deficits Are Detectable in Premanifest Huntington Disease Using the California Verbal Learning Test

Contact:

McKenna Williams

Graduate Student

mckennaewilliams@gmail.com

661-414-4836

3923 Centre Street, Unit H

San Diego, CA 92103

United States

McKenna E. Williams ${ }^{1}$, Heather M. Holden ${ }^{2}$, Savanna M. Tierney ${ }^{3}$, Lisa V. Graves ${ }^{1,2}$, Dean C. Delis ${ }^{2,4}$, Jody Corey-Bloom ${ }^{2,5}$, Paul E. Gilbert ${ }^{1,2}$

${ }^{1}$ Department of Psychology, San Diego State University, San Diego, CA, USA; ${ }^{2}$ Joint Doctoral Program in Clinical Psychology, San Diego State University/University of California San Diego, San Diego/La Jolla, CA, USA; ${ }^{3}$ Department of Psychology, University of Houston, Houston, TX, USA; ${ }^{4}$ Department of Psychiatry, University of California San Diego School of Medicine, La Jolla, CA, USA; ${ }^{5}$ Department of Neurosciences, University of California San Diego, La Jolla, CA, USA

Verbal memory impairment is well documented in Huntington disease (HD), although studies examining memory processes in premanifest HD have yielded mixed results. Many studies have utilized a comparison group comprising individuals who are children of a parent diagnosed with HD but do not carry the expanded CAG repeat themselves. Cognitive performance in this group may be complicated by a host of psychiatric symptoms, including anxiety, depression, survivor's guilt, and caregiver burden. The present study aimed to characterize verbal learning and memory performance in premanifest HD (PreHD) using a large sample of premanifest gene-carrying individuals $(n=46)$ and a demographically similar comparison group of healthy adults with no family history or genetic risk of $\mathrm{HD}(n=60)$. Eleven variables from the California Verbal Learning Test-II (CVLT-II) that assess immediate and delayed recall, recognition, learning characteristics, errors, and retention were selected for analysis. CVLT-II recall and recognition discriminability measures also were examined. PreHD participants demonstrated worse performance on learning trials, short and long delayed recall, recognition, and intrusions, suggesting that subtle declines in these memory processes may be evident prior to clinical diagnosis of HD. Additionally, the PreHD group performed worse than healthy adults on most recall discriminability measures, as well as Novel Recognition Discriminability. The current study supports the utility of the CVLT-II in detecting subtle episodic memory changes during the premanifest stage of HD.

\section{1}

Gait and Balance Dual-Task Performance in Individuals with Huntington Disease

Contact:

Gregory Youdan

$\mathrm{PhD}$ Student

gay2104@tc.columbia.edu

646-339-4497

201 West 83rd St. Apt 1D

New York, NY 10024

United States

Gregory Youdan Jr. ${ }^{1}$, Nora Fritz ${ }^{2}$, Lisa Muratori ${ }^{3}$, Monica Busse ${ }^{4}$, Lori Quinn ${ }^{1}$

${ }^{1}$ Teachers College, Columbia University, New York, NY, USA; ${ }^{2}$ Wayne State University, Detroit, MI, USA; ${ }^{3}$ Stony Brook University, Stony Brook, NY, USA; ${ }^{4}$ Cardiff University, Cardiff, Wales, UK

Background: Huntington disease (HD) is an inherited, neurodegenerative disorder resulting in motor and cognitive impairments. Minimal research exists on the performance effects of dual tasks in HD, especially examining prioritization strategies.

Aim: The aim is to characterize complex dual-task performance during standing and walking conditions and to examine prioritization strategies used by individuals with HD.

Methods: This cross-sectional, multicenter observational study included 37 participants with early-to-mid-stage $\mathrm{HD}$ (mean $\pm \mathrm{SD}$ age, $53.5 \pm 10.5$; 20M/17F; TFC, $10.8 \pm 2.0$ ) and 15 healthy controls (age, 52.4 \pm 13.1 ; $9 \mathrm{M} / 6 \mathrm{~F})$. While wearing inertial sensor participants performed standing balance tasks (feet apart, feet together, and standing on foam) and walking tasks (Timed Up and Go and $7 \mathrm{~m}$ Walk) under both single and dual-task conditions (Stroop Test was administered during standing tasks; reciting every letter and every other letter of the alphabet was administered during walking tasks). Total sway area (standing tasks), gait speed (walking tasks), and total correct responses (cognitive tasks) were used to calculate dual-task costs and effects. One-way ANOVAs were used to examine differences between groups.

Results: Preliminary results showed significant between-group differences $(p<0.03)$ for total sway area, gait speed, and correct response rate under both single and dual-task conditions. There was a significant interaction effect for standing feet apart and TUG conditions, where performance on dual-task conditions were more impaired for HD participants $(p<0.04)$. The majority of HD participants exhibited mutual interference 
with cognitive and motor decline in dual-task conditions, but a few HD participants showed strong cognitive prioritization.

Conclusions: Preliminary results suggest that TUG time and postural sway during standing feet apart appear to be the most sensitive measures to address dual-task impairments in this population. Evaluation of individual dual task effects may provide additional insights into the nature of motor and cognitive phenotypes in HD.

\section{2}

Exploring Emotion Regulation and Emotion Recognition in People with Presymptomatic Huntington Disease: the Role of Emotional Awareness

\section{Contact:}

Nicolò Zarotti, MD

n.zarotti@lancaster.ac.uk

+4407922330167

233 St. Pauls City Lofts

Sheffield S12LL

United Kingdom

Nicolò Zarotti ${ }^{1}$, Jane Simpson ${ }^{1}$, Ian Fletcher ${ }^{1}$, Ferdinando Squitieri ${ }^{2}$, Simone Migliore ${ }^{2}$

${ }^{1}$ Division of Health Research, Faculty of Health and Medicine, Lancaster University, Lancaster, UK; ${ }^{2}$ Huntington and Rare Diseases Unit at IRCCS Casa Sollievo della Sofferenza Research Hospital, San Giovanni Rotondo, Italy

Interest in the role of both emotion regulation and recognition in our understanding of mental health has been steadily increasing, especially in people with chronic illness who also have psychological difficulties. One illness that belongs to this category is Huntington disease. Huntington disease (HD) is a chronic neurodegenerative disorder that can cause a number of cognitive and psychological difficulties, including emotion recognition deficits, even before the onset of the symptoms required to make a formal diagnosis. Despite the lack of definite evidence, recent studies have suggested that deficits of emotion regulation and recognition may be expected to play a pivotal role in the early cognitive manifestations of HD.

In this study, we hypothesized that the ability to regulate emotions can be impaired in people with presymptomatic HD, and that such impairment may be associated with a deficit of emotion recognition. To test this, an online survey was carried out with 117 English and Italian-speaking people with presymptomatic HD, compared with 217 controls matched for age and education.

The results suggest that, in presymptomatic participants, emotion regulation and emotion recognition are generally not significantly impaired, and no significant relationships between performances on the two constructs were observed. However, a specific impairment in emotional awareness (a subscale on the Difficulties in Emotion Regulation Scale (DERS)) was observed, which appears to be enhanced by the co-occurrence of depressive symptoms, even at a subclinical level. Consequently, it is suggested that difficulties in emotional awareness may represent a precursor of more general emotion recognition impairments, which only become apparent as the disease reaches a more symptomatic level. Clinical implications of the findings are discussed and directions for future research are proposed.

\section{3}

Clinical Development of VX15 Anti-semaphorin 4D Antibody as a Potential Treatment for Huntington Disease

\section{Contact:}

Terrence Fisher, $\mathrm{PhD}$

Sr. Director, Clinical and Translational Science

tfisher@vaccinex.com

585-271-2700 x71

1895 Mt. Hope Ave.

Rochester, NY 14620

United States

\section{Presenter:}

Maurice Zauderer

President and CEO

mzauderer@vaccinex.com

585-271-2700 x107

1895 Mt. Hope Ave.

Rochester, NY 14620

United States

\section{Zauderer, J. Leonard, T. Fisher, E. Evans, E. Smith, A. Feigin}

Vaccinex, Inc., Rochester, NY, USA (all except A. Feigin)

Andy Feigin is representing the Huntington Study Group, SIGNAL investigators, and coordinators

Chronic inflammation, neuronal degeneration, and disruption of the bloodbrain barrier (BBB) are believed to play an important role in neuroinflammatory and neurodegenerative diseases. Semaphorin 4D (SEMA4D) and its high-affinity receptor, Plexin B1 (PLXNB1), are expressed on brain neural, endothelial, and inflammatory cells. SEMA4D signaling through PLXNB1 triggers activation of inflammatory glial cells, inhibits migration and differentiation of glial progenitor cells that can repair damage to myelin, and disrupts endothelial tight junctions that are required for the integrity of the BBB. We have demonstrated in several preclinical models, including HD transgenic mice, that antibody neutralization of SEMA4D ameliorates neurodegenerative processes. This was the rationale for initiating SIGNAL, a randomized (1 to 1), double-blind, placebocontrolled phase 2 study of treatment with VX15/2503 anti-SEMA4D antibody, pepinemab, enrolling patients with HD.

Two study cohorts were planned: Cohorts A and B. Enrolled patients were treated with monthly IV infusions of either pepinemab or matching placebo. Cohort A enrolled 36 patients, and the analysis of these data was completed in April 2017. Data from this analysis were used to inform the design of Cohort B. Currently, Cohort B of the SIGNAL clinical trial is enrolling 160 early manifest and 80 prodromal HD subjects. The study endpoints include quantitative cognitive (HD-CAB) and motor assessments (Q-Motor and TMS), and functional and patient reported outcomes, as well as imaging measures of change in energy metabolism and volumetric MRI in defined brain regions.

The SIGNAL trial experience to date has found no concerning safety signals and participant retention and compliance with the study protocol compares favorably with prior experience in other HD studies. We will report on progress in enrollment of Cohort B and will review the interpretation and significance of some of the key observations from Cohort A.

94

Defining the Roles of Autophagy Cargo Receptors in the Turnover of Huntingtin Protein and Huntington Disease Pathogenesis

Contact:

Sheng Zhang

Assistant Professor

sheng.zhang@uth.tmc.edu

713-500-3493

1825 Pressler Street 
The University of Texas McGovern Medical School at Houston Houston, TX 77030

United States

Boli $\mathrm{Hu}^{1}$, Shiyu $\mathrm{Xu}^{1}$, Gang $\mathrm{Li}^{1}$, Antonio Tito ${ }^{1}$, Erin Stimming ${ }^{3}$, Sheng Zhang ${ }^{1,2,4}$

${ }^{1}$ The Brown Foundation Institute of Molecular Medicine, Houston, TX, USA $;{ }^{2}$ Department of Neurobiology and Anatomy, USA $;{ }^{3}$ Department of Neurology, HDSA Center of Excellence McGovern Medical School at the University of Texas Health Science Center at Houston (UTHealth), Houston, TX, USA; ${ }^{4}$ Programs in Human and Molecular Genetics and Neuroscience, The University of Texas MD Anderson Cancer Center, UTHealth Graduate School of Biomedical Sciences (MD Anderson UTHealth GSBS), Houston, TX, USA

Mutated huntingtin (HTT) protein is the primary cause of Huntington disease (HD). It is thus important to elucidate the cellular pathways regulating the turnover of endogenous HTT, especially the mechanisms that can selectively target mutant HTT protein. Existing studies support that macroautophagy and the autophagy-lysosomal (ALP) pathways, a major cellular clearance and protective mechanism against misfolded proteins and aggregates, are effective in eliminating mutant HTT and suppressing its toxicity. However, general regulators of autophagy and ALP pathways, such as those targeting mTOR and TFEB, do not confer substrate specificity and may lead to adverse effects associated with systematic activation. Selective autophagy is a subtype of macroautophagy that requires cargo receptors (e.g., SQSTM1/p62, NBR1, and Optineurin) to recognize specific targets including misfolding proteins for their sequestration in the autophagosome and their eventual degradation through ALP, thereby representing an attractive mechanism for targeted clearance of mutant HTT. However, an increasing number of cargo receptors, often with redundant functions, are being identified. Further, HTT itself also functions in autophagy, including its association with p62 and its role as a scaffold for selective autophagy. Together, they implicate a potentially complicated regulatory network in autophagic degradation of mutant HTT. To investigate the roles of selective autophagy in HTT turnover, we are systematically generating knockout cell lines for a panel of cargo receptors and examining their effect on the levels of normal and mutant HTT. Our preliminary data suggest differential roles of cargo receptors in basal autophagy and HTT turnover.

Lay Summary: How to effectively remove mutated huntingtin (HTT), the protein responsible for Huntington disease (HD), is central for targeted therapy against the disease. Macroautophagy and the autophagy-lysosomal (ALP) pathways are major cellular self-defense mechanisms against toxic proteins including mutant HTT inside the cell.

\section{5}

\section{Identification and Functional Characterization of Hap40 Homolog in Drosophila}

\section{Contact:}

Sheng Zhang

Assistant Professor

sheng.zhang@uth.tmc.edu

713-500-3493

1825 Pressler Street

The University of Texas McGovern Medical School at Houston

Houston, TX, 77030

United States

Shiyu $\mathrm{Xu}^{1}$, Gang $\mathrm{Li}^{1}$, Boli $\mathrm{Hu}^{1}$, Antonio Tito ${ }^{1}$, Erin Stimming ${ }^{3}$, Sheng Zhang ${ }^{1,2,4}$

${ }^{1}$ The Brown Foundation Institute of Molecular Medicine, Houston, TX, USA; ${ }^{2}$ Department of Neurobiology and Anatomy, USA; ${ }^{3}$ Department of
Neurology, HDSA Center of Excellence, USA McGovern Medical School at the University of Texas Health Science Center at Houston (UTHealth), Houston, TX, USA; ${ }^{4}$ Programs in Human and Molecular Genetics and Neuroscience, The University of Texas MD Anderson Cancer Center UTHealth Graduate School of Biomedical Sciences (MD Anderson UTHealth GSBS), Houston, TX, USA

Huntington disease (HD) is caused by an abnormal expansion of the glutamine tract (polyQ) in huntingtin (HTT) protein. As a large-scaffold protein with numerous reported binding partners (HAPs), HTT is implicated in a growing list of cellular processes from vesicular transport to transcription to autophagy. However, little is known how these diverse functions are integrated through HTT and how HTT itself is regulated. Converging evidence support that HAP40 is a central regulator of HTT. HAP40 was originally isolated from rat and mouse brains as the "most significantly correlated" partner of endogenous HTT protein and binds HTT in a stochastic 1:1 M ratio. Recently, HAP40 was found to stabilize the structure of HTT, converting it from a conformational heterogeneity status to a well-defined globular structure. Importantly, in both primary fibroblasts and striatal tissues, an $\sim 10$-fold increase of the levels of endogenous HAP40 were observed in samples from HD patients as compared with healthy controls, implicating a pathogenic role in HD. Despite the strong biochemical and structural evidence, there is no reported functional evaluation of HAP40 in any physiological setting; its role on HTT functions, mutant HTT toxicity, and HD pathogenesis remains unclear. In a proteomic study for the HTT homolog (dHtt) in Drosophila, we isolated a novel $\sim 40 \mathrm{kDa}$ protein as the strongest binding partner for endogenous $\mathrm{dHtt}$. This $40-\mathrm{kD}$ a protein has significant sequence homology with mammalian HAP40 and is renamed as dHap40 (Drosophila Hap40). The coevolution of Hap40 and HTT in evolutionarily distant species from flies to humans not only supports the functional importance of HAP40 in HTT regulation but also establishes Drosophila as a relevant genetic model to evaluate the physiological and pathological roles of HAP40. To this end, we have created several dhap40 knockout mutants and also transgenic flies for dHap40 overexpression, and currently are characterizing the resulting phenotypes. Given the complex pathogenic mechanisms underlying HD, a clear understanding of central HTT regulators such as HAP40 is essential for "HTT-lowering" and other novel therapeutic strategies against HD.

97

Hypothalamic Suppression of Mutant Huntingtin Improves the Phenotype of BACHD Rats and Restores Proteins Involved in Energy Metabolism

\section{Contact:}

Hoa Huu Phuc Nguyen, MD

hoa.nguyen@med.uni-tuebingen.de

+497071-29 72288

Calwerstr. 7

72076 Tübingen

Germany

Janice Stricker-Shaver ${ }^{1,2}$, Benedikt Fabry ${ }^{1,2}$, Libo Yu-Taeger ${ }^{1,2}$, Elisabeth Singer ${ }^{1,2}$, Lisa Stanek $^{3}$, Carsten Calaminus ${ }^{4}$, Bernd J. Pichler $^{4}$, Lamya S. Shihabuddin ${ }^{3}$, Olaf Riess ${ }^{1,2}$, Huu Phuc Nguyen ${ }^{1,2}$

${ }^{1}$ Huntington Disease Research Group, Institute of Medical Genetics and Applied Genomics, University of Tübingen, 72076 Tübingen, Germany, ${ }^{2}$ Centre for Rare Diseases, University of Tübingen, 72076 Tübingen, Germany, ${ }^{3}$ Rare Disease Unit, Neuroscience, Sanofi-Genzyme, Framingham, MA, USA, ${ }^{4}$ Werner Siemens Imaging Center, Department of Preclinical Imaging and Radiopharmacy, University of Tuebingen, 72076 Tübingen, Germany 
In Huntington disease (HD), the negative impacts of metabolic dysfunctions heavily affect the quality of life of patients. As energy metabolism is centrally regulated by the hypothalamus via a number of proteins, we hypothesize that mutant huntingtin (mHTT) disrupts selective proteins resulting in metabolic disturbances.

To investigate the disruptions of energy metabolism caused by mHTT in the hypothalamus, we injected a viral-mediated microRNA that could downregulate both wild-type and mutant HTT into the hypothalamus of BACHD rats. To compare the effects of mHTT downregulation at the time of intervention, BACHD rats were treated at 1 and 6 months of age, which correspond to the early disease and symptomatic stages, respectively. Wild-type littermates treated with vector formulation buffer and BACHD rats treated with an empty vector were included as control groups. Longitudinal assessments on several behavioral aspects were conducted after treatment for 5 months and compared with the shamtreated wild-type rats and empty-vector-treated BACHD rats. At the end of the study, brain tissues were harvested for protein analyses.

Behavioral and postmortem analyses showed that improvements were not limited to metabolic but also motor phenotypes at both therapeutic time points when mHTT was downregulated. Protein expressions of neuropeptide Y receptor 5 (NPY5R), orexin receptor 1 (OX1R), and leptin receptor were significantly altered in at least one of the disease stages in the hypothalamus of the empty-vector-treated BACHD rats when compared with the wild-type littermates. Downregulation of mHTT in the hypothalamus restored the protein expressions of NPY5R, OX1R, and leptin receptor in the hypothalamus to respective levels as in the wild-type littermates.

Our results indicate that suppression of mHTT in the hypothalamus can be an effective approach for improving the phenotype and for restoring the perturbed neuroendocrine axis that regulates energy metabolism.

\section{8}

\section{The Social and Educational Impact of Attending a Huntington Disease (HD)-Specific Camp}

\section{Contact:}

Chandler Swope

Youth Worker

chandler@hdyo.org

202-674-4848

528 Kennedy St NW \#302

Washington, DC 20011

United States

\section{Presenter Name:}

Catherine Martin

Executive Director

catherine@hdyo.org

202-674-4848

528 Kennedy St NW \#302

Washington, DC 20011

United States

\section{Chandler Swope ${ }^{1}$, Matthew Ellison ${ }^{2}$, Catherine Martin $^{3}$ \\ ${ }^{1}$ Washington, DC, USA; ${ }^{2}$ Bedford, England, UK; ${ }^{3}$ Glasgow, Scotland, UK}

Background: There are many camps available to support young people who are currently suffering from symptoms from an illness or condition, but little availability of camps for those with parents suffering from an illness in which the young people are at risk for developing the same illness. The Huntington's Disease Youth Organization (HDYO) has hosted eight camps around the world, over the course of 5 years for young people impacted by
Huntington disease (HD). The aim of these camps is to provide the camper respite, a space for peer and professional support, and access to education that is age and stage appropriate.

Case History: Campers overwhelmingly spoke about camp helping them feel less isolated and more in control of their journey with HD. Over $50 \%$ of campers in our study (only ten campers completed all pre- and postsurveys) stated they had more specific knowledge about HD; there was also a statistically significant increase in the campers' sense of social support and an increase in positive sense of self-esteem (Kavanaugh et al. 2017).

Conclusion: There are still limited data on camps for young people who are at-risk of $\mathrm{HD}$, but early information points to the existence of these camps as being hugely important for healthy outcomes. Furthermore, feeling educated about the disease and research about the disease leads to young people feeling empowered and less afraid of the disease. Campers can also get practical information to help them make critical decisions (testing, family planning, symptom management, etc.) for their own journey with HD.

\section{9}

Clues from the Atlantic Squid Loligo pealeii Reveal Novel Mechanisms Underlying Axonal Pathology in Huntington Disease

\section{Contact:}

Gerardo Morfini

Associate Professor

gmorfini@uic.edu

312-996-6869

Department of Anatomy and Cell Biology

808S Woods St Rm\#568

Chicago, IL 60612

United States

Gerardo Morfini, Mercedes Priego, Norma Hernandez, Scott Brady

Department of Anatomy and Cell Biology, University of Illinois at Chicago, Chicago, IL 60612, USA (all authors)

A mechanistic understanding of the pathogenic processes operating in Huntington disease (HD) would provide key insights for the development of therapeutic strategies aimed at preserving neural connectivity in this disease. Within this context, degeneration of axons projecting from specific neuronal populations within the basal ganglia represents an early and critical pathogenic event in HD. However, specific mechanisms and molecular components linking mutant huntingtin (mhtt) to axonal pathology remain largely unknown.

Our previous work using the isolated squid axoplasm preparation showed that mhtt promotes deficits in axonal transport, a cellular process critically needed for appropriate maintenance of synaptic function and axonal connectivity. Extending these findings, additional studies revealed that $m h t t$ inhibits axonal transport through a mechanism involving activation of the protein kinase JNK3 and phosphorylation of the motor protein conventional kinesin. However, whether JNK3 contributes to mhtt-induced neuropathology in vivo remained unknown.

Results from genetic experiments in both squid axoplasm and the R6/2 mouse model of HD will be presented, revealing both a novel gain of function mechanism associated with polyglutamine expansion in htt and a major contribution of JNK3 to the development of neuropathological features in the R6/2 model. Collectively, our findings suggest that JNK3 may represent a novel therapeutic target to preserve neuronal connectivity in HD. 
100

Intrinsic Oligodendroglial Dysfunction Underlies Primary Myelination Abnormalities and Contributes to Behavioral Manifestations in Huntington Disease

\section{Contact:}

Mahmoud Pouladi

Assistant Professor

pouladi@tlgm.a-star.edu.sg

656-407-0700

8A Biomedical Grove

Immunos Level 5

Singapore 138648

Singapore

Costanza Ferrari Bardile ${ }^{1}$, Marta Garcia-Miralles ${ }^{1}$, Nicholas Caron ${ }^{2}$, Nirmala Arul Rayan ${ }^{3}$, Sarah Langley ${ }^{4}$, Nathan Harmston ${ }^{4}$, Roy Tang Yi $\mathrm{Teo}^{1}$, Prabhakar S ${ }^{3}$, Enrico Petretto ${ }^{4}$, Michael R. Hayden ${ }^{1,2,5}$, Mahmoud A. Pouladi ${ }^{1,5}$

${ }^{1}$ Translational Laboratory in Genetic Medicine, Agency for Science, Technology and Research, Singapore (A*STAR), Singapore; ${ }^{2}$ Centre for Molecular Medicine and Therapeutics, Child and Family Research Institute, University of British Columbia, Vancouver, BC, Canada; ${ }^{3}$ Genome Institute of Singapore, Agency for Science, Technology and Research, Singapore, Singapore; ${ }^{4}$ Systemic Genetics Group, Duke-NUS Medical School, Centre for Computational Biology, 8 College Road, Singapore, Singapore; ${ }^{5}$ Department of Medicine, Yong Loo Lin School of Medicine, National University of Singapore, Singapore, Singapore

White matter atrophy and myelin abnormalities appear to be significant features of Huntington disease (HD). Although white matter atrophy appears very early in the disease course, the molecular factors that underline myelination deficits in HD are poorly understood. Here, we hypothesize that intrinsic mutant huntingtin (mHTT)-mediated effects in oligodendroglia contribute to myelin deficits and behavioral manifestations in HD. To test this hypothesis, we crossed the BACHD mouse model of $\mathrm{HD}$, which expresses full-length human mHTT and mimics many of the behavioral and neuropathological features of the human condition, to NG2-Cre mice in order to silence mHTT specifically in oligodendroglia. Electron microscopy was used to analyze myelin fibers of the corpus callosum (CC) at 1 and 12 months of age, and a battery of behavioral tests were performed to evaluate motor and psychiatric-like phenotypes in mice. Furthermore, to gain insights into the molecular mechanisms underlying the oligodendrocyte dysfunction observed in HD mice, we performed RNA-seq analysis on the CC of mice at 1 month of age. We show that selective removal of mHTT from oligodendroglia rescues the deficits in thickness and compactness of myelin sheaths and improves certain aspects of behavioral dysfunction in HD mice. Mechanistically, we show that mHTT promotes PRC2 activity and disrupts the expression of key genes associated with myelination. Our findings suggest that epigenetic mechanisms are involved in the intrinsic oligodendroglia dysfunction in $\mathrm{HD}$ and contribute to myelin abnormalities and behavioral manifestations of the disease.

\section{1}

Development of a Novel Patient-Reported Outcome Measure for Huntington Disease: the Huntington Disease Health Index (HD-HI) Study

\section{Contact:}

Alistair Glidden

Clinical Research Coordinator

alistair.glidden@chet.rochester.edu
585-275-2039

265 Crittenden Blvd CU 420694

Rochester, NY 14642

United States

Alistair M. Glidden ${ }^{1}$, Olivia S. Brumfield ${ }^{1}$, Elizabeth A. Luebbe ${ }^{1}$, Christine E. Zizzi ${ }^{1}$, Molly J. Elson ${ }^{2}$, Steven B. Goldenthal ${ }^{3}$, Ray Dorsey $^{1}$, Chad R. Heatwole ${ }^{1}$

${ }^{1}$ University of Rochester, Rochester, NY, USA; ${ }^{2}$ Emory University, Atlanta, GA, USA; ${ }^{3}$ University of Michigan, Ann Arbor, MI, USA

Background: Properly validated patient-reported outcome measures for use in Huntington disease (HD) clinical trials are lacking. Health indices that reflect patient outcomes have been implemented in clinical trials for other inherited disorders, including myotonic dystrophy, facioscapulohumeral muscular dystrophy, Charcot-Marie-Tooth disorder, and spinal muscular atrophy.

Objective: This study aims to develop a patient-reported outcome measure that reflects the most important HD symptoms as assessed by affected individuals.

Methods: From 40 interviews ( $N=20$ with HD; $N=20$ caregivers), we analyzed 2082 quotes regarding the symptomatic burden of HD. We subsequently developed a survey with 216 potential symptoms representing 15 symptomatic themes. We electronically distributed the survey to 389 participants, comprising individuals with HD $(N=96)$, prodromal HD $(N=60)$, and caregivers $(N=233)$. Participant responses determined the prevalence and severity ( 0 to 4$)$ of each symptom, which were then multiplied to generate a population impact factor. Based on these scores and other factors (e.g., responsiveness, language), we developed the initial version of the Huntington Disease Health Index (HD-HI). We subsequently performed cognitive interviews with 15 patients with HD to determine the usability of the final instrument and rest-retest reliability testing using a weighted kappa statistic for each question using 15 HD patients. Comparisons were performed between baseline (paper format) and 2-week (paper format) responses and between 2-week (paper format) and 4-week (computer format) responses.

Results: Respondents in the beta testing and test-retest reliability phases hailed from 23 states and were 53\% women, 50\% college-educated, and had an average age of 48 years. Those with manifest HD (78\% of respondents) had an average self-reported Total Functional Capacity score of 8.8 and an average CAG repeat length of 43. Participants during beta testing took an average of $17 \mathrm{~min}$ to complete the HD-HI. The wording of one question was modified based on patient input. Analysis of the test-retest reliability data from week 0 and 2 shows that all but two symptomatic questions were reliable over a 2 -week period. Analysis of the 2- and 4week test-retest data demonstrated near equivalence of patients using the paper and computer version of the instrument.

Conclusions: We have developed a novel multifaceted disease-specific patient-reported health index that can be incorporated into HD therapeutic trials in the immediate future.

Sponsorship: Research support provided by NJ Cure and the Huntington Study Group.

102

Assessment of Caregiver Burden in Huntington Disease

\section{Contact:}

Margaret Yu, MD

margaret.yu@northwestern.edu

312-823-9620

345W Fullerton Parkway Apt 2003

Chicago, IL 60614

United States 
Margaret Yu, Kenny Tan, Kimberly Koloms, Danny Bega

Northwestern University Feinberg School of Medicine, Chicago, IL, USA (all authors)

Background: Huntington disease (HD) is a rare neurodegenerative disease associated with progressive cognitive and motor disability and loss of independence. The caregivers of HD patients are at high risk for burnout and often suffer reduced quality of life (QoL). Identifying at-risk caregivers and the factors that lead to the most reduction in QoL is crucial for early intervention.

Objectives: We aim to identify and characterize burden among HD caregivers to see what aspects of HD caregiving are associated with the greatest burden and have the highest impact on QoL.

Methods: Caregivers of established patients with HD seen at Northwestern University HD clinic from January to April of 2017 were approached about participation in a caregiver burden survey, which included the Modified Caregiver Strain Index (MCSI), the Huntington's Disease Quality of Life Battery for Carers Short Form (HD-SF), and several demographic questions. Total Functional Capacity (TFC) of the associated patient was also recorded. Simple linear regression analysis was utilized to assess the effect of variables with suspected impact on caregiver burden to both MCSI and HD-SF.

Results: Twenty out of twenty caregivers who were approached responded. A lower TFC score was associated with greater caregiver burden on MCSI $(r=-0.46 ; p=0.04)$ but not on HD-SF $(r=0.002$; $p=0.994)$. The only other variable that correlated with burden was being the main carer on MCSI ( $p$ value $=0.04$, limited by only two participants in the nonmain carer group). Interstudy correlation coefficient between HD-SF and MCSI was not statistically significant $(r=-0.157 ; p=$ 0.5076).

Conclusions: Given the lack of significant findings, either our population is not representative or the scales for assessing for burden in this population are unreliable or fail to capture what drives caregiver burden. This area requires more research and further investigation into screening caregivers for early intervention and in determining the appropriate scales for doing so.

\section{3}

\section{Quantification of HD Participant Interest in Tele-visits, Wearables, and Sensors}

\section{Contact:}

Jody Goldstein

Co-Chair HSG Executive Coordinator Council

Jody.Goldstein@ chet.rochester.edu

760-845-2105

265 Crittenden Blvd., CU 420694, Suite 2312

Rochester, NY 14642-0694

United States

Jody Goldstein $^{1}$, Elaine Sperin ${ }^{2}$, Amy Chesire ${ }^{1}$, Hope Heller ${ }^{3}$, Jenna Smith $^{4}$, Julia Spears ${ }^{5}$, Greg Suter ${ }^{6}$, G; Brandi Crews ${ }^{7}$, Elise Kayson ${ }^{1}$ (on behalf of the HSG Executive Coordinator Council).

${ }^{1}$ University of Rochester, Rochester, NY, USA; ${ }^{2}$ Emory University, Atlanta, GA, USA; ${ }^{3}$ Georgetown University, Washington, DC, USA; ${ }^{4}$ University of Alabama at Birmingham, Birmingham, AL, USA; ${ }^{5}$ University of Toledo Medical Center, Toledo, OH, USA; ${ }^{6}$ Greg Suter, Hereditary Neurological Disease Centre, Wichita, KS, USA; ${ }^{7}$ University of Cincinnati/Cincinnati Children's Hospital, Cincinnati, OH, USA

Objective: The key aim of the survey was to evaluate Huntington disease (HD) patients' and families' interest and willingness to participate in televisits and to use wearables and sensors in clinical studies.
Background: Previous experience has shown that clinical study participation is limited by close proximity to a research site. A tele-visit (TV) could bring a clinical study to participants, allowing more individuals to participate while potentially lessening the travel and time burden of inperson research visits. Wearables and sensors could provide continuous data collection versus a one-time assessment of a participant's HD status. Currently, research participants travel to research sites to participate in clinical studies. Clinical study designs will begin offering an experience that is new to HD studies, called a TV. A TV will bring the clinical study to participants. Research study staff will connect with participants remotely, at home or at a convenient location, or over a video visit (like Skype) to conduct study activities. Activities such as blood work, blood pressure, ECGs, and monitoring of study drug use, etc., may require a nurse to visit a participants' home or another convenient location. Studies will introduce wearables and sensors, such as iPhones, watches, etc., that can be worn on the body to capture movement and other symptoms associated with HD. Device data will be compared with scores generated by neurologists.

Design/Methods: A 13-question survey was designed to ask participants about: (1) Internet usage and TV experience, (2) interest and willingness to participate in a TV or device study, and (3) who completed the survey. Results/Conclusion: One hundred and eighteen patients (72\%) and caregivers/families (38\%) completed the survey. Eighty-one percent were willing/very willing to participate in tele-visit and device studies; $94 \%$ had Internet access and were comfortable with a nurse coming to the home; $100 \%$ had participated in video messaging; and $89 \%$ were willing to wear devices $24 \mathrm{~h} /$ day. New research assessment tools may change the design of HD clinical studies. Tele-visits may allow more individuals to participate in clinical studies. Further questionnaires could ascertain whether participants are willing to complete all study visits via TV versus a mix of both in-person and TV and could enable the evaluation of the perceived benefits and drawbacks to these types of study visits.

\section{4}

\section{Quality of Life and Reproductive Choices of People with Huntington} Disease

\section{Contact:}

David LeVan

Associate Professor

levan005@gannon.edu

814-602-7801

109 University Square

Erie, PA 16541

United States

David LeVan, Juliet Corrigan, Kathryn Fillman, Rachael Mack, Zoe Maslowski

Gannon University, Erie, PA, USA (all authors)

Background: Huntington disease (HD) has a 50\% inheritance rate and combined with progressive physical, emotional, and psychosocial challenges directly impacts quality of life (QOL), family dynamics, and reproductive decision making of people who are diagnosed with HD. In addition to coping with the disease, people with HD experience an additional burden of knowing how and when to communicate with spouses and family members about their attitudes and beliefs toward reproductive decision making. Objective: The purpose of this qualitative phenomenological study was to gain a deeper understanding of attitudes, beliefs, and personal experiences about reproductive decision making and its influence on QOL from the perspective of those with HD and their spouses.

Methods: Two married people with HD, their spouses, and one single person with HD participated in a focus group discussion to allow for open 
conversation between participants and researchers. Guided interview questions and probing were utilized to facilitate participants' points of view.

Results: Through thematic and conceptual qualitative analysis, five general themes about QOL and reproductive decision making emerged from the focus group discussion and included Longing for a Cure, Family Ignorance, Freedom of Choice, Spreading Awareness, and Education.

Conclusion: The findings of this small study can provide opportunities for shared experiences and insight for other people with HD who are making the same decisions. A greater understanding of QOL and reproductive choices of people with HD can improve advocacy and treatment provided by family members and healthcare providers.

\section{5}

Problems that Bother Parkinson Disease Patients: Framework for a Patient-Reported Natural History of Parkinson and Huntington Diseases

\section{Contact:}

Ira Shoulson

Founder, Gray Matter Technologies LLC; Adjunct Professor, University of Rochester

ira@irashoulson.org

585-733-8110

4473 Gulf of Mexico Dr.

Longboat Key, FL 34228

United States

Ira Shoulson ${ }^{1,2}$, Lakshmi Arbatti ${ }^{1}$, Andrew Nguyen ${ }^{1,3}$, David Standaert ${ }^{4}$, Connie Marras ${ }^{5}$, Caroline Tanner ${ }^{6}$

${ }^{1}$ Grey Matter Technologies LLC, Sarasota, FL, USA; ${ }^{2}$ University of Rochester, Rochester, NY, USA; ${ }^{3}$ University of San Francisco, CA, USA; ${ }^{4}$ University of Alabama at Birmingham, AL, USA; ${ }^{5}$ University Health Network of Toronto, Toronto, Canada; ${ }^{6}$ University of California, San Francisco, CA, USA

No systematic research has been performed to characterize the course of neurological disease as reported directly by affected patients. We are examining an extraordinary learning set of 5000 Parkinson disease (PD) patients who volunteered on the FoxInsight.org research platform to answer, in their own words, two research questions in the form of the Patient Reported Outcome of Problems (PD-PROP): 1) What bothers you the most about your PD? and 2) In what way does this problem bother you by affecting your daily functioning? Participants were also asked to report what else bothers them, for up to five bothersome problems. We analyzed and curated their verbatim replies using natural language processing (NLP) and machine learning (ML) approaches. Four motor (tremor, rigidity, bradykinesia, and postural instability) and six nonmotor (sleep, fatigue, cognition, mood, pain, and constipation) domains were classified with respect to age and 010 years since diagnosis. Verbatim reports were analyzed independently by three movement disorder specialists to further characterize domain terms. Motor and nonmotor problems increased in frequency with duration of PD and to a lesser extent with the age of the patient. Nonmotor problems $(\sim 40 \%)$ were nearly as bothersome as motor problems $(\sim 60 \%)$. The verbatim patient reports and application of NLP/ML techniques provide a preliminary, cross-sectional natural history of PD from the relevant perspective of patients- to be further informed as the cohort grows to $\sim 100,000$, as longitudinal data accrue, and as more granular analytic techniques are applied. The PD-PROP was modeled after the Huntington Disease PROP (HD-PROP), which has been applied as an exploratory outcome in the REACH2HD clinical trial (Ann Neurol 80(S20), 2016).
Ongoing curation of the PD-PROP, along with expanded verbatim reports by those affected by $\mathrm{HD}$, will refine the utility of the HDPROP as a tool for clinical care and research.

107

Greater Caregiver's Burden in Unawareness of Huntington Disease

\section{Contact:}

Pierre Wibawa, MD

pierre.wibawa@gmail.com

$+61426288411$

Royal Melbourne Hospital, Neuropsychiatry Unit

300 Grattan Street, Parkville

Melbourne 3050

Australia

Pierre Wibawa ${ }^{1,2,3}$, Peter Panegyres ${ }^{1}$, Daniel Rock ${ }^{4}$, Rachel Zombor ${ }^{1}$, Brad Hayhow ${ }^{1}$, Joseph Lee ${ }^{1}$, Jacenta Abbott ${ }^{1}$, Milan Dragovic ${ }^{2,4}$, Sergio E. Starkstein ${ }^{4,5}$

${ }^{1}$ Neurosciences Unit, North Metropolitan Health Service-Mental Health, Perth, Australia; ${ }^{2}$ Clinical Research Centre, North Metropolitan Health Service-Mental Health, Perth, Australia; ${ }^{3}$ Neuropsychiatry Unit, Royal Melbourne Hospital, Melbourne, Australia; ${ }^{4}$ School of Psychiatry and Clinical Neurosciences, the University of Western Australia, Perth, Australia; ${ }^{5}$ Fremantle Hospital, Department of Health, Perth, Australia

Background: Unawareness of one's obvious disease manifestation, or anosognosia, is estimated to occur in up to one-in-two Huntington disease (HD) patients. While unaware patients seem to have lower incidents of mental health and quality of life issues (McCusker and Loy, Tremor Other Hyperkinet Mov (N Y) 4:257, 2014), little is known about whether unawareness impacts on caregivers' perceptions of burden.

Objectives: This study aims to evaluate any associations between unawareness in HD patients' and caregivers' burden.

Methods: This is a cross-sectional study in a state-wide neurosciences outpatient clinic. Clinician, patient, and caregiver complete the Anosognosia Scale (Deckel and Morrison, Arch Clin Neuropsychol 11(4):295-302, 1996), Novak's Caregiver Burden Inventory (CBI), and Zarit Burden Interview (ZBI). Unified Huntington's Disease Rating Scale: motor, behavior and cognitive, Mini-Mental State Examination, and functional assessment are also performed.

Results: Ten $(26.3 \%)$ out of 38 patients were assessed as "unaware." Significantly higher caregivers' burden was observed in both CBI (aware vs unaware, mean scores, 13.8 vs 30.6; $p<0.005$ ) and ZBI (aware vs unaware, mean scores, 22.1 vs 38.4; $p<0.001$ ). There were no significant group differences in the patients' and caregivers' demographics. However, group differences were observed in executive function: Stroop Interference, Animal Verbal Fluency, and Symbol Digit Modalities Test (mean differences of 16, 6.9 , and 12.9, respectively; $p<0.05$ ).

Conclusions: Unawareness is common and associated with greater caregivers' burden in HD. Although the prognosis of unawareness remains unknown, education and support for caregivers should be provided incipiently.

References:

1. McCusker, E. \& Loy, C. T. (2014). The many facets of unawareness in Huntington disease. Tremor Other Hyperkinet Mov (N Y), 4, 257. doi:https://doi.org/10.7916/D8FJ2FD3

2. Deckel, A. W. \& Morrison, D. (1996). Evidence of a neurologically based "denial of illness" in patients with Huntington's disease. Arch Clin Neuropsychol, 11(4), 295-302. 
108

Reward-Dependent Cognition in Presymptomatic and Symptomatic Huntington Disease

Contact:

Madeleine Sharp

Assistant Professor, Department of Neurology and Neurosurgery, McGill University

madeleine.sharp@mcgill.ca

514-398-5174

3801 Rue University

Montreal Neurological Institute, Rm 256

Montreal QC H2B 3A4

Canada

Madeleine E. Sharp ${ }^{1}$, Paula Wasserman ${ }^{2}$, Karen S. Marder*2, Daphna Shohamy*3

${ }^{1}$ Department of Neurology and Neurosurgery, McGill University, Montreal, QC, Canada; ${ }^{2}$ Department of Neurology, Columbia University Medical Center, New York, NY, USA; ${ }^{3}$ Department of Psychology and Mind, Brain Behavior Institute, Columbia University, New York, NY, USA

Striatal neurons are impacted earliest in the course of Huntington disease, and striatal atrophy is a sensitive marker of disease progression. It is also known that patients with Huntington disease, even in the earliest stages of the disease, have subtle cognitive impairments, but their relation to striatal function remains poorly understood. Remarkably, despite the extensive literature demonstrating the crucial role of the striatum in signaling reward, there has been very little focus on studying reward-related cognitive processes in patients with Huntington disease. Here, we aimed to fill this gap by measuring reward learning and reward-guided memory in presymptomatic Huntington patients $(n=36)$, symptomatic patients $(n=$ $34)$, and healthy controls $(n=18)$. We adapted a probabilistic reinforcement learning task in which participants gradually learned to associate stimuli with reinforcing outcomes. On each trial, at the time of feedback, participants were also shown a trial-unique image that, by its category assignment, indicated whether they were correct or incorrect on that trial. This allowed us to measure the influence of reward on two processes: reinforcement learning and reward-modulated episodic memory.

As predicted, results show that learning and memory were worse in the symptomatic Huntington patients. Importantly, the same measures were also impaired in presymptomatic patients, demonstrating sensitivity of these tasks to early stages of the disease. We additionally found a relationship between task performance and estimated disease burden, which is calculated using the mutation length (number of CAG trinucleotide repeats) and age, among presymptomatic and symptomatic patients. Specifically, increased disease burden was strongly associated with worse reinforcement learning. The degree to which learning and memory are impaired was also associated with measures of executive function. These results suggest that specific striatal-dependent cognitive mechanisms are impaired and that measures of reward-guided cognition could be used as a marker of disease, even in the earliest stages.

\section{9}

Explaining Trajectory for the Influence of Genetic Prevalence on Development of Psychopathologies Among Students at Risk of Huntington Disease

\section{Contact:}

Faiza Afridi

Lecturer faiza.afridi10@gmail.com

92334-886-0404

Peshawar 25000

Pakistan

Faiza Khan Afridi ${ }^{1}$, Jamil A. Malik ${ }^{2}$

${ }^{1}$ Department of Psychology, Shaheed Benazir Bhutto Women University, Peshawar, Pakistan; ${ }^{2}$ National Institute of Psychology, Quaid-i-Azam University, Islamabad, Pakistan

Background: Prevalence of Huntington disease (HD) in the family is a major contributor to the development of depression, anxiety, and stress of children due to increased concern of being at risk.

Aims: We investigated the underlying mechanisms of the genetic prevalence of HD in developing psychopathologies through increased amotivation, which further decreases belongingness and causes a decline in the quality of social relations that results in a decrease in general selfefficacy.

Method: Participants included students who are at risk of developing HD due to genetic influence. A total of $N=30$ participants with age ranging from 14 to 27 years with mean \pm SD $(20.97 \pm 3.25)$ including $63 \%$ females were included in the study. Along with demographic information, data were collected on standardized instruments to measure amotivation, belongingness, depression, anxiety, stress, and quality of social relations. Genetic prevalence was measured at the ordinal level with one repressing prevalence in one generation (grandparents only), two depicting two generations (i.e., grandparents and parents or siblings), and three showing prevalence in all three generations (i.e., grandparents, parents, and siblings).

Results: Genetic influence was associated with an increase in amotivation $(r=0.38 ; p<0.05)$, as well as psychological distress $(r=$ $0.48 ; p<0.01)$. An increase in amotivation was further associated with decreased belongingness $(r=-0.40 ; p<0.05)$ and general self-efficacy $(r=-0.58 ; p<0.01)$ and increased psychological distress $(r=0.53$; $p<0.01$ ). Belongingness is positively associated with quality of social relations $(r=0.46 ; p<0.01)$ and negatively associated with psychological distress $(r=-0.42 ; p<0.01)$. Quality of social relations was further positively associated with general self-efficacy $(r=0.50 ; p<0.01)$, which has a strong negative association with psychological distress $(r=0.75$; $p<0.01$ ). Model testing was conducted in Mplus 8 , which resulted in a good fit.

Conclusions: It is concluded that genetic influence increased psychological distress both directly and indirectly by decreasing the role of positive factors such as belongingness, quality of social relations, and general selfefficacy and increasing the role of negative factors such as amotivation.

\section{0}

Juvenile Onset Huntington Disease: a 15-Year Observational Study Delineates Clinical Presentation, Disease Progression, and Brain Abnormalities

\section{Contact:}

Ferdinando Squitieri, MD, PhD

ferdinandosquitieri@yahoo.it

+393396425285

CSS-Mendel Institute (IRCCS Casa Sollievo della Sofferenza Research Hospital)-Viale Regina Margherita, 261

00198 Rome, Italy

Caterina Fusilli ${ }^{1}$, Simone Migliore ${ }^{1}$, Tommaso Mazza ${ }^{1}$, Federica Consoli $^{1}$, Alessandro De Luca ${ }^{1}$, Gaetano Barbagallo ${ }^{2}$, Andrea Ciammola $^{3}$, Emilia Mabel Gatto ${ }^{4}$, Martin Cesarini ${ }^{4}$, Jose Luis Etcheverry ${ }^{4}$, Virginia Parisi ${ }^{4}$, Musallam Al-Oraimi ${ }^{5}$, Salma Al-Harrasi ${ }^{5}$, 
Qasem Al-Salmi ${ }^{5}$, Massimo Marano ${ }^{6}$, Jean-Paul Gerard Vonsattel ${ }^{7}$, Umberto Sabatini ${ }^{2}$, Georg Bernhard Landwehrmeyer ${ }^{8}$, and Ferdinando Squitieri ${ }^{1}$

${ }^{1}$ IRCCS Casa Sollievo della Sofferenza (Rome CSS-Mendel), San Giovanni Rotondo, Italy; ${ }^{2}$ Department of Neuroradiology, University Magna Graecia, Catanzaro, Italy; ${ }^{3}$ Department of Neurology and Laboratory of Neuroscience, IRCCS Istituto Auxologico Italiano, Milan, Italy; ${ }^{4}$ Departments of Movement Disorders, Instituto Neurociencias de Buenos Aires (INEBA), and Neurology, Sanatorio de la Trinidad Miter, Buenos Aires, Argentina; ${ }^{5}$ National Genetic Centre, Royal Hospital, Ministry of Health, Muscat, Sultanate of Oman; ${ }^{6}$ Italian League for Research on Huntington and Related Diseases Foundation (LIRH), Rome, Italy; ${ }^{7}$ The New York Brain Bank, Columbia University, New York, USA ${ }^{8}$ Abteilung Neurologie, Universitätsklinik Ulm, Ulm, Germany

Background: Symptoms of juvenile HD (JHD) start $\leq 20$ years of age, with large-sized mutations generally associated with the rare adolescent or infantile variant. There is a lack of published literature regarding JHD symptomatology, disease course, and brain pathology, and how these compare with adult-onset HD (AHD).

Methods: We extracted retrospective data (June 2003 to March 2017) from 36 patients with ascertained JHD (psychiatric and/or motor symptoms $\leq 20$ years of age) and retrospectively analyzed symptoms at their onset and over time. Clinical data were also analyzed to compare disease progression in these patients versus those with AHD. Radiology and postmortem specimen images from patients with $\geq 80$ cytosine-adenineguanine $(\mathrm{CAG})$ repeats were examined, where available, and lifespan of deceased patients with JHD and AHD were compared.

Findings: Children with large-sized ( $\geq 80 \mathrm{CAG}$ repeats) and unstable CAG expansions progressed more severely than AHD patients, had a reduced life span, and showed early specific brain pathologies with early reduced striatal volume and relatively preserved cortical and white matter structures over time. Of note, we found the same abnormalities in an autoptic brain obtained from a child with infantile age at onset and a large-sized mutation $>80$ CAG repeats.

Interpretation: JHD with large-sized mutations $(n=10)$ differ from other juvenile $(n=26)$ and adult-onset $(n=97)$ cohorts. Notably distinct are symptom presentation, disease progression, brain pathology, and life span, justifying therapeutic timing and strategies different from adults and a radical revision of the current disease classification.

\section{1}

Did the Announcement of the IONIS-HTTRx Trial Completion Influence New Patient Visit Patterns in a HD Clinic?

\section{Contact:}

Jennifer Klapper

Clinic Coordinator/Research Nurse

jennifer.klapper@uphs.upenn.edu

215-829-5176

330S. 9th St

3rd Floor

Philadelphia, PA 19107

United States

Jennifer Klapper, Tanya Bardakjian, Pedro Gonzalez-Alegre

University of Pennsylvania, Philadelphia, PA, USA (all authors)

Background: Subjects at risk for HD may not have sought predictive testing due to the lack of disease-modifying therapies. The research findings announced for Ionis-HTTRx (RG6042) in December 2017 led to significant optimism and encouragement in HD families. We hypothesized that this would lead to a change in the pattern of patients and families contacting our HDSA Center of Excellence for evaluation which, in turn, could affect optimal clinical care in HD.

Objective: Our objective was to determine if there was a change in new patient referrals to the HDSA Center of Excellence at the University of Pennsylvania in the 6 months preceding and following the announcement of the successful completion of the Ionis-HTTRx (RG6042) trial in December 2017.

Methods: We reviewed electronic records for types of office visits in the 6 months prior to 31 December 2017 (interval 1) and in the 6 months following (interval 2), tallying the number of new patient visits (NPV), Genetic Counseling for Predictive Testing (GC-PT), and return patient visits (RPV).

Results: There were $22 \mathrm{NPV}$ in interval 1 , with six presenting for GC-PT. In interval 2, there were $37 \mathrm{NPV}$, with 21 presenting for GC-PT. Other NPV (not for GC-PT) and RPV remained constant in both intervals.

Summary: Potential increases in new patient visit volume after the December 2017 Ionis-HTTRx (RG6042) announcement, as witnessed at our HD Center, may require adjustments by HD clinics to handle increased new patient visit referrals, genetic counseling, and predictive testing. We suggest this may also include exploring patient goals for care and including education during the genetic counseling process on the differences between successful safety and efficacy trials in order to establish realistic patient and family-centered expectations for care.

\section{2}

\section{NOI Huntington - the Italian HD Youth-Driven Engagement Project}

\section{Contact:}

Barbara D'alessio

barbara.dalessio@lirh.it

$+393272870465$

Via Varese, 31

00185 Rome

Italy

\section{Barbara D’Alessio $^{1}$, Simone Migliore ${ }^{1,2}$, Alessia Migliore ${ }^{1}$}

${ }^{1}$ LIRH Foundation, Rome, Italy, ${ }^{2}$ IRCCS Casa Sollievo della Sofferenza Hospital (Rome-CSS Mendel), San Giovanni Rotondo, Italy

Background: The Italian League for Research on Huntington and Related Diseases Foundation (LIRH Foundation) is the Italian not-forprofit organization entirely committed to fostering research and improving care for patients with HD. Thanks to continuous relationships with hundreds of affected families, we know very well that teenagers and young adults are the weakest members of the HD community. For this reason, we accepted to support a youth-driven project, called NOIHuntington, which was borne from the idea of a small group of young at-risk and affected boys and girls.

Objective: NOI Huntington aims to offer to the children and young adults who are affected by or at risk for HD and who are living in Italy the opportunity to know each other, to share their personal experiences, to share a positive approach to the disease, and to contribute to HD research. Methods: The LIRH Foundation hosted the first meeting of the funding group at its headquarters in Rome. The meeting also hosted a neuropsychologist. LIRH financially helped boys and girls to create a video presentation of the project as a way to encourage other young people to join. Results: The video presentation was first launched on 2 December 2017. It had more than 14,000 views in less than 10 days. A Facebook page, an Instagram page, and a YouTube channel were launched. In addition, the website is about to be launched. Currently, the group comprises 40 young people from 10 different Italian regions out of 20 regions. 
Conclusions: The funding group wishes to become a national network. It is willing to collaborate with other HD youth organizations at national and international levels and is strongly interested in supporting research on HD and JHD.

113

Sleep Disorder in Huntington Disease: Prevalence, Risk Factors, and Pharmacological Treatment Trends as Derived from the Enroll-HD Database

Contact:

Sandra Kostyk, MD, PhD

sandra.kostyk@osumc.edu

614-293-4969

7th Floor

Columbus, OH 43210

United States

Kelin L. Wheaton, Allison M. Daley, Junan Li, Karl Obrietan, Kari R. Hoyt, Sandra K. Kostyk

The Ohio State University, Columbus, Ohio, USA (all authors)

Background: Although involuntary movements are a hallmark of Huntington disease (HD), nonmotor symptoms also profoundly affect individuals with HD. Disruption of the sleep/wake cycle may be an early nonmotor feature of HD. Sleep disruption has negative effects on quality of life and may contribute to progressive decline in cognitive, psychological, and emotional wellbeing. The prevalence, risk factors, and pharmacological treatment strategies for sleep disruption in HD have not been thoroughly explored.

Methods: We utilized the Enroll-HD database to quantify the number of individuals in whom "disorders of initiating and maintaining sleep" had been recorded as a comorbidity. Subjects were divided into predefined subgroups: (1) genotype negative ( $n=2100)$, (2) premanifest $(n=1862)$, and (3) manifest $(n=4753)$ HD. For those with documented sleep disorders $(n=595)$, clinical variables were selected to statistically determine if any covariates could be considered "risk factors" for development of sleep disorders. Medication profiles were probed for frequency of prescribed sleep aids.

Results: Compared with genotype-negative controls, individuals with premanifest HD had a twofold increase in the probability of having a documented sleep disorder, whereas those with manifest disease had a fourfold increased probability $(p<0.0001)$. When only North American subjects were considered, this association nearly doubled for the manifest subgroup (eightfold increase). Increased body mass index (BMI) and high anxiety, apathy, and depression severity scores were risk factors associated with presence of sleep disorders for both HD groups. Antidepressants were the most frequently prescribed sleep aid for those with $\mathrm{HD}$ with trazodone and mirtazapine prescribed in more than $90 \%$ of these cases.

Conclusions: A significant increase in prevalence of sleep disorders was detected in individuals with premanifest and manifest HD compared with genotype-negative controls. Further exploration of the nature of sleep disorders in HD may help identify opportunities for improved interventions and enhanced quality of life.

Skeletal Muscle Mitochondrial Dysfunction but Not Mitochondrial DNA Damage Contributes to Motor Deficit and Body Weight Decline in the R6/2 Mouse Model of Huntington Disease

\section{Contact:}

Sylvette Ayala-Pena

Associate Professor

sylvette.ayala@upr.edu

787-758-2525

Room A-335 Main Building

Department of Pharmacology and Toxicology

San Juan, PR 00936-5067

United States

Sulay Rivera-Sánchez ${ }^{1}$, Karina Acevedo-Torres ${ }^{1}$, Joan Ballista Hernández ${ }^{2}$, Lourdes Colón Ortíz ${ }^{2}$, Sharon Fonseca-Williams ${ }^{3}$, María del R Castro ${ }^{2}$, Alvin Ayala-Colón ${ }^{2}$, Giselle Barreto-Torres ${ }^{3}$, Rebecca Parodi-Rullán ${ }^{3}$, Sabzali Javadov ${ }^{3}$, Carlos A. Torres-Ramos ${ }^{3}$, Cynthia T. McMurray $^{4}$, Sylvette Ayala-Peña ${ }^{2}$

${ }^{1}$ Puerto Rico Center for Inherited Diseases, Department of Pediatrics, San Juan, PR 00936-5067, USA; ${ }^{2}$ Department of Pharmacology and Toxicology, University of Puerto Rico Medical Sciences Campus, P.O. Box 365067, San Juan, PR 00936-5067, USA; ${ }^{3}$ Department of Physiology and Biophysics, University of Puerto Rico Medical Sciences Campus, P.O. Box 365067, San Juan, PR 00936-5067, USA; ${ }^{4}$ Life Sciences Division, Lawrence Berkeley National Laboratory, 1 Cyclotron Rd., Berkeley, CA 94720, USA

Background: Studies in Huntington disease (HD) patients and animal models of HD suggest that skeletal muscle (SKM) pathology and mitochondrial dysfunction may contribute to the motor deficit and body weight loss characteristic of HD. Damage to the mitochondrial DNA (mtDNA) has been reported as a precipitating event leading to mitochondrial dysfunction and neurodegeneration in HD. However, it is unknown whether signals originating from the SKM contribute to the motor and body weight phenotype in $\mathrm{HD}$.

Methods/Results: We tested whether damage to the mtDNA, mitochondrial respiratory dysfunction, or both contribute to the motor and body weight phenotype in two mouse models of HD: the R6/2 transgenic and the homozygous $H d h Q 150^{(++)}$knock-in mice. We show a 2 -fold increase $(p<0.001)$ in mtDNA lesion frequency and a $34 \%$ decrease $(p<0.001)$ in mtDNA molecules in the gastrocnemius of HD animals versus wild-type (WT) mice. Treatment with idebenone, a mitochondrial electron carrier and antioxidant, resulted in a 1.4-fold $(p<0.02), 1.6$-fold, and 2.0-fold $(p<0.02)$ increase in motor performance in 7-, 9-, and 13week-old mice versus age-matched vehicle-treated mice, and in a significant $(p<0.003)$ improvement in body weight $(\sim 6 \mathrm{~g}$ compared with vehicle-treated R6/2) in 13-week-old mice. Interestingly, idebenoneinduced genes that mediate mitochondrial biogenesis, restored the respiratory control index to WT levels, and increased state 3 complex II and complex IV respiration rates by $73 \%(p<0.05)$ and $41 \%(p<0.05)$, respectively, compared with untreated R6/2 mice, but did not prevent the increase in mtDNA lesions or mtDNA depletion in R6/2 mice.

Conclusions: (1) SKM mitochondrial dysfunction, but not damage or depletion of mtDNA, might significantly contribute to motor/body weight deficits in HD. (2) Increased mitochondrial biogenesis may be involved in SKM-mitochondrial adaptations in response to idebenone. (3) Targeting SKM-mitochondrial capacity is an attractive therapeutic approach for delaying disease progression by improving motor function and body weight.

\section{5}

Maximal and Submaximal Exercise Capacity in Prodromal and Early Manifest Huntington Disease

Contact:

Ashwini K. Rao

Associate Professor of Rehabilitation and Regenerative Medicine 
akr7@cumc.columbia.edu

917-279-4923

710 West 168th Street

Room 1110

New York, NY 10032

United States

Ashwini K. Rao ${ }^{1,2}$, Simone Norris ${ }^{1}$, Paula Wasserman ${ }^{2}$, K.S. Marder ${ }^{2,3}$

${ }^{1}$ Rehabilitation and Regenerative Medicine (Physical Therapy), New York, NY, USA; ${ }^{2}$ GH Sergievsky Center, Columbia University, New York, NY, USA; ${ }^{3}$ Neurology, Psychiatry, Taub Institute, Columbia University, New York, NY, USA

Introduction: We examined maximal and submaximal exercise capacity in prodromal and manifest Huntington disease and compared their performance with matched healthy controls.

Methods: We tested ten pHD (mean, 43.2 years), ten mHD (mean, 52.5 years), and seven controls (mean, 46.6 years). Maximal exercise capacity was measured with the cardiopulmonary exercise test using a recumbent bicycle. Primary outcomes were heart rate, oxygen consumption, and workload. Body composition was measured using Bioelectrical Impedance Analysis. Primary outcomes were fat mass, fat-free mass, and total body water. Submaximal exercise was tested using the 6-min walk test on a 25-m course. Distance walked in each minute was computed. Height, weight, BMI, fat mass, fat-free mass, total body water, and age were analyzed using one-way ANOVA. Maximal and submaximal exercise data were analyzed using factorial ANOVA. Regression analyses were used to determine if age, BMI, and fat-free mass were predictors of maximal and submaximal exercise capacity.

Results: Age, height, weight, BMI, fat mass, fat-free mass, and total body water (Hubbard equations) were similar across groups $(p>0.05)$. During maximal exercise, heart rate and workload was lowest for manifest HD subjects at $40 \%, 50 \%$, and $75 \%$ and maximal capacity $(p<0.05)$. Peak $\mathrm{VO} 2$ reserve was lower for prodromal and manifest $\mathrm{HD}(p<0.01)$. The 6min walk test distance was lowest for manifest HD subjects $(p=0.024)$. There was a decrement in distance walked per minute for prodromal and manifest HD. Maximal and submaximal capacity were not predicted by age, BMI, or fat-free mass $(p>0.05)$

Conclusion: Reduction in maximal and sub-maximal exercise capacity is seen early in manifest HD. There is evidence of weakness (reduced 6MWT distance) in manifest HD and evidence of fatigue (reduction in distance from minute 1 to minute 6) in prodromal and manifest HD. Reduced exercise capacity was not explained by age, BMI, or fat-free mass.

\section{6}

Human Neural Stem Cells as a Developmental Candidate for Huntington Disease

\section{Contact:}

Leslie Thompson, $\mathrm{PhD}$

Chancellor's Professor

lmthomps@uci.edu

949824-6756

Department of Neurobiology and Behavior

3214 Biosci 3

University of California

Irvine, CA 92697-4545

United States

J.C. Reidling ${ }^{1}$, S.M. Holley ${ }^{2}$, C. Cepeda ${ }^{2}$, A. Relaño-Ginés ${ }^{3}$, J. Ochaba ${ }^{4}$, C. Moore ${ }^{5}$, B. Fury ${ }^{6}$, A. Lau ${ }^{7}$, S. Yeung ${ }^{1}$, J.S. Steffan ${ }^{9}$, M. BlurtonJones $^{10}$, C.K. Meshul ${ }^{11}$, G. Bauer ${ }^{6}$, M.F. Chesselet ${ }^{3}$, M.S. Levine ${ }^{12}$, C.N. Svendsen ${ }^{13}$, L.M. Thompson ${ }^{14}$
${ }^{1}$ Institute for Memory Impairment and Neurological Disorders, University of California, Irvine, CA, USA; ${ }^{2}$ Intellectual and Developmental Disabilities Research Center, Semel Institute for Neuroscience and Human Behavior, David Geffen School of Medicine, University of California, Los Angeles, CA, USA; ${ }^{3}$ Department of Neurology, David Geffen School of Medicine, University of California, Los Angeles, CA, USA; ${ }^{4}$ Department of Neurobiology \& Behavior, University of California, Irvine, CA, USA; ${ }^{5}$ Portland VA Medical Center, Portland, OR, USA; ${ }^{6}$ Institute for Regenerative Cures, University of California, Davis, CA, USA; ${ }^{7}$ Department of Psychiatry \& Human Behavior, University of California, Irvine, CA, USA; ${ }^{8}$ Department of Neurobiology \& Behavior, University of California, Irvine, CA, USA; ${ }^{9}$ Department of Neurobiology \& Behavior, University of California, Irvine, CA, USA; ${ }^{10}$ Sue and Bill Gross Stem Cell Center, University of California, Irvine, CA, USA; ${ }^{11}$ Portland VA Medical Center, Portland, OR, USA; Oregon Health \& Science University, Department of Behavioral Neuroscience, Portland, OR, USA; ${ }^{12}$ Intellectual and Developmental Disabilities Research Center, Semel Institute for Neuroscience and Human Behavior, David Geffen School of Medicine, University of California, Los Angeles, CA, USA;

${ }^{13}$ Brain Research Institute, David Geffen School of Medicine, University of California, Los Angeles, CA 90095, USA; ${ }^{14}$ Board of Governors Regenerative Medicine Institute, Cedars-Sinai Medical Center, Los Angeles, California, USA

No disease-modifying treatments yet are in clinical use for HD patients that can either delay onset or modify disease progression. We are exploring cell-based approaches for treatment of HD given their potential to modulate neuropathology in other degenerative brain diseases, as suggested by promising preclinical studies in Parkinson's disease (PD) and HD. Cell-based approaches are promising as a treatment option for Huntington disease (HD) with great potential to modulate pathology in a complex tissue such as brain. We have evaluated transplantation of pluripotent hESC-based derivatives (e.g., non-MSC) and have demonstrated robust efficacy from transplantation of GMP-compatible human neural stem cell (hNSC) lines in multiple HD-modeled mice, supporting feasibility for this approach. This strategy provides behavioral efficacy and shows improved electrophysiological measurements and decreased aberrant mutant Huntington protein accumulation. Notably, these cells form synaptic contacts, suggesting the possibility for improvements in brain neuron connections. We are currently moving forward with INDenabling safety studies in preclinical models of HD. In parallel, we have performed a preliminary study using a stem-cell-based therapy currently in a phase I clinical trial for ALS. These cells, developed by Clive Svendsen at Cedars-Sinai Medical Center, are neural precursor cells (CNS10-NPC) that are banked and readily available for the clinic. The preliminary study showed efficacy in the rapidly progressing HDmodeled mice (R6/2). We are currently performing an HSG-sponsored study to examine the long-term benefit of transplanted hNPCs in zQ175 knock-in HD mice to address fundamental questions relating to survival, differentiation, migration, and safety of transplanted cells, as well as the mechanism of action to advance the potential of bringing this cell-based therapeutic for HD to the clinic. We hope to show that a single surgical transplantation of CNS10-NPCs may be efficacious in HD subjects.

\section{7}

\section{Identification of Biomarkers for Early-Detection and Monitoring of Huntington Disease}

\section{Contact:}

David Salzman

President and CEO

david.salzman@srnalytics.com

201-404-0265 
AstraZeneca BioHub Incubator

35 Gatehouse Drive

Waltham, MA 02451

United States

David W. Salzman ${ }^{1}$, Joli Bregu ${ }^{1}$, Nathan S. Ray ${ }^{2}$, Richard H. Myers ${ }^{1,2}$

${ }^{1}$ Boston University Medical School, Department of Neurology, 72 East Concord Street, Boston, MA, 02118, USA; ${ }^{2}$ sRNAlytics Inc. AstraZeneca BioHub Incubator, 35 Gatehouse Drive, Waltham, MA, 02451, USA

MicroRNAs (miRNAs) are a class of noncoding RNAs that control gene expression at the posttranscriptional level. The current library of human miRNAs consists of 2588 annotated genes. However, these small RNAs are subject to variability during and after biogenesis resulting in an array of sequence variants for each annotated miRNA, called isomiRs. Each of these isomiRs can have templated and/or nontemplated variants at the $5^{\prime}$ and/or $3^{\prime}$ end that impacts the biological function and localization of these genes. sRNAlytics developed a computational platform that combines next-generation sequencing with big data analytics and machine learning to identify biomarkers of disease. Using our platform and data from Hoss et al. (2015), Reed et al. (2017), and other sources, we identified isomiRs that are uniquely expressed in the frontal cortex of Huntington patients but not in $>2500$ non-Huntington controls. IsomiRs were validated using targeted RT-qPCR in 124 independently collected samples from 64 brains and 55 cerebrospinal fluid samples. Our results showed that these biomarkers are 1) only expressed in individuals carrying the Huntingtin gene mutation, 2) can be detected up to 20 years prior to symptomatic onset, and 3) increase with abundance over time. sRNAlytics filed these biomarkers as a Context of Use for early detection and disease monitoring through the FDA Biomarker Qualification Program. We believe these Huntington disease-specific isomiRs will enable the discovery of new drug targets and provide an efficacious biomarker to facilitate on-going drug development programs.

\section{8}

Contrasting Psychiatric Symptoms of Huntington Disease and Frontotemporal Dementia

Contact:

Edward Huey

edh2126@cumc.columbia.edu

212-305-1134

Taub Institute, Columbia University Medical Center

630 West 168th Street, P\&S Box 16

New York, NY 10032

United States

Rachel Fremont, Hannah Silverman, Masood Manoochehri, H. Blair Simpson, Karen Marder, and Edward D. Huey

Taub Institute, Columbia University Medical Center, New York, NY, USA (all authors)

Neurodegenerative disorders are devastating illnesses that are accompanied by significant neurological impairment. More recently, psychiatric dysfunction resulting from these same illnesses has become more appreciated. Certain psychiatric manifestations of dementing illness are associated with worse outcomes, higher distress to family members, and increased rates of institutionalization. Two syndromes with significant documented psychiatric symptoms are Huntington disease (HD) and frontotemporal dementia
(FTD, which includes behavioral variant FTD (bvFTD) and semantic variant primary progressive aphasia (svPPA)). An important symptom domain for both HD and FTD is repetitive thoughts and behaviors. While initial studies have been performed characterizing the rates of psychiatric disorders in HD and FTD, there has been little work comparing atrophy patterns and psychiatric symptoms between them. To this end, we have developed and piloted the Neurodegeneration-Associated Psychiatric Syndromes (NAPS) study to directly address this question. As part of the NAPS study, we are characterizing and contrasting repetitive thoughts and behaviors in HD and FTD using the Yale-Brown Obsessive-Compulsive Scale (YBOCS), the Structured Clinical Interview (DSM), and the DSM Field Trial Cross-Cutting Symptom measure. Here, we report preliminary findings from this study suggesting that FTD variants and HD present with unique constellations of psychiatric symptoms. Focusing on repetitive thoughts and behaviors, we demonstrate that different neurodegenerative disorders can have remarkably different psychiatric manifestations, which we hypothesize correlate with distinct atrophy patterns in the brain. We hope that understanding the different neural substrates of psychiatric symptoms in these disorders through the NAPS study will help in the development of targeted anatomically based treatments such as TMS.

\section{9}

\section{Pelvic Organ Dysfunction in Huntington Disease Patients}

\section{Contact:}

Alice B. Liu, MPH

Research Associate, University of Washington

abliu@uw.edu

Mentors:

1. Claire Yang, MD, Chief of Urology, Harborview Medical Center, Professor of Urology, University of Washington, Adjunct Professor of Neurology, University of Washington.

2. Suman Jayadev, MD, Assistant Professor of Neurology, University of Washington, Director, Neurogenetics Clinic, University of Washington, Director, University of Washington Huntington Disease Center of Excellence.

Huntington disease (HD) is an autosomal dominant neurodegenerative disease caused by an increased number of tri-nucleotide repeats in the coding region of the Huntington gene. The triad of motor, cognitive, and psychological symptoms that characterizes the disease comprises most of the literature on this subject to date. Atrophy and neuronal loss in the striatum and other brain areas are the known neuro-pathologic causes for symptoms. Previous understanding of HD identifies little association between the disease and the development of pelvic organ dysfunction - encompassing bladder, bowel, and sexual functioning - which has a significant impact on patient quality of life $(\mathrm{QoL})$. However, there is very limited information on the prevalence of these symptoms in the context of the Huntington disease (HD) population and of their impacts on patients' lives.

As such, the aim of this research is to build a patient-centered, online questionnaire platform to better understand the prevalence of pelvic organ symptoms, impacts on QOL, and patients' experiences related to these symptoms. The questionnaire will be distributed to a diverse population of HD patients across the USA through partnering HD research, education, and advocacy organizations. The results from this questionnaire study will be used to generate scientific literature contributing to better understanding of the impact of pelvic organ dysfunction on HD patients. In addition, these data will be used in tandem with subject-matter expertise to generate HDrelevant patient, family, and caregiver education materials to help manage these symptoms; these educational materials will be distributed through online resources and through partnering HD organizations. 